\title{
ALMGREN-TYPE MONOTONICITY METHODS FOR THE CLASSIFICATION OF BEHAVIOR AT CORNERS OF SOLUTIONS TO SEMILINEAR ELLIPTIC EQUATIONS
}

\author{
VERONICA FELLI AND ALBERTO FERRERO
}

\begin{abstract}
A monotonicity approach to the study of the asymptotic behavior near corners of solutions to semilinear elliptic equations in domains with a conical boundary point is discussed. The presence of logarithms in the first term of the asymptotic expansion is excluded for boundary profiles sufficiently close to straight conical surfaces.
\end{abstract}

\section{INTRODUCTION}

This paper presents a monotonicity approach to the study of the asymptotic behavior near corners of solutions to semilinear elliptic equations

$$
-\operatorname{div}(A(x) \nabla u(x))+\mathbf{b}(x) \cdot \nabla u(x)-\frac{V\left(\frac{x}{|x|}\right)}{|x|^{2}} u(x)=h(x) u(x)+f(x, u(x))
$$

in a domain $\Omega \subset \mathbb{R}^{N}, N \geqslant 2$, having the origin as a conical boundary point. The coefficients b : $\Omega \rightarrow \mathbb{R}^{N}$ and $h: \Omega \rightarrow \mathbb{R}$ are possibly singular at 0 but satisfy suitable decaying conditions (see assumptions (12) and (13) below) which make the corresponding terms negligible with respect to the homogeneity of the operator, while the nonlinearity $f$ has at most critical growth in the Sobolev sense (see assumptions (16-17)).

Due to their own theoretical interest and their numerical application to convergence analysis of finite element approximations, regularity and asymptotics near corners of solutions to linear elliptic equations in domains with piecewise boundary have been intensively studied and a large literature has been devoted to this subject (see [3], the monographs [6] and [17, Chapter 3], the surveys $[11,13]$, and the references therein). Some early contributions in this field date back to papers $[14,22]$ which use methods based on conformal maps and integral representation to derive asymptotic expansions for harmonic functions at a common endpoint of two analytic arcs delimiting the 2-dimensional simply connected domain; such asymptotic development excludes the presence of logarithmic terms for irrational values of $\alpha$, where $\alpha \pi$ is the opening of the corner. On the other hand, a simple example shows that, if $\alpha=\frac{n}{m}, n, m \in \mathbb{N} \backslash\{0\}$, is a rational number, then there exist harmonic functions with smooth trace on the boundary of the domain but having a logarithmic term in the leading part of the asymptotic expansion: it is sufficient to consider the classical example $u(x, y)=\Im\left(z^{m} \log z\right), z=x+i y$, in the domain

$$
\left\{(x, y)=(r \cos \theta, r \sin \theta) \in \mathbb{R}^{2}: r>0, \theta \in(0, \pi / m)\right\} .
$$

In [14, Theorem 3.4] logarithmic terms are excluded in the leading expansion term in the case of homogeneous boundary conditions also for rational values of $\alpha$.

Related results for semilinear Dirichlet problems on plane domains with corners were obtained in $[12,23]$; see also [15] for the study of existence and nonexistence of solutions to singular semilinear elliptic equations on cone-like domains. We mention that edge asymptotics (which is naturally related to corner asymptotics) is investigated in [5] (see also the references therein).

In the spirit of the paper [20], which provides asymptotics of positive solutions to $p$-Laplace equations with forcing terms and non-homogeneous boundary conditions on straight $N$-dimensional

V. Felli is partially supported by the PRIN2009 grant "Critical Point Theory and Perturbative Methods for Nonlinear Differential Equations". A. Ferrero is partially supported by the PRIN2008 grant "Aspetti geometrici delle equazioni alle derivate parziali e questioni connesse".

2010 Mathematics Subject Classification. 35J15, 35J61, 35J75, 35A16.

Keywords. Almgren monotonicity formula, semilinear elliptic equations, conical boundary points. 
cones, we mean to describe the rate and the shape of solutions to (1) near corners of domains which are perturbations of cones, by relating them to the eigenvalues and the eigenfunctions of a limit operator on the spherical cap measuring the opening of the vertex. The method this paper is proposing for valuating the asymptotic behavior of solutions to (1) is based on the monotonicity method introduced by Almgren [2] in 1979 and then extended by Garofalo and Lin [10] to elliptic operators with variable coefficients in order to prove unique continuation properties. Monotonicity methods were recently used in $[7,8,9]$ to prove not only unique continuation but also precise asymptotics near singularities of solutions to linear and semilinear elliptic equations with singular potentials, by extracting such precious information from the behavior of the quotient associated with the Lagrangian energy. Almgren type formulas were also used in [1] to prove unique continuation at the boundary; the diffeomorphic deformation of the domain performed in [1] (see also [18]) to get rid of the boundary contributions inspires our construction of the equivalent problem (37) in section 2, for which a monotonicity formula is derived in section 5 .

As a byproduct of our asymptotic analysis we also obtain a unique continuation principle for solutions of (1) vanishing with infinite order at the conical point of the boundary.

The strengths of the monotonicity formula approach are described in the note [9]: they essentially rely in the sharpness of the asymptotics derived, in the possibility of allowing quite general perturbing potentials, and in the unified approach to linear and nonlinear equations.

In subsection 1.1 we introduce notation and assumptions needed to state our main result Theorem 1.1.

1.1. Assumptions and main results. For $N \geqslant 2$, let $\varphi: \mathbb{R}^{N-1} \rightarrow \mathbb{R}$ and $g: \mathbb{S}^{N-2} \rightarrow \mathbb{R}$ such that, for some $\delta>0$,

$$
\begin{aligned}
& \varphi(0)=0, \quad \varphi \in C^{2}\left(\mathbb{R}^{N-1} \backslash\{0\}\right), \\
& g \in C^{1}\left(\mathbb{S}^{N-2}\right) \quad \text { if } N \geqslant 3, \\
& \sup _{\nu \in \mathbb{S}^{N-2}}\left|\frac{\varphi(t \nu)}{t}-g(\nu)\right|=O\left(t^{\delta}\right) \quad \text { as } t \rightarrow 0^{+}, \\
& \left\{\begin{array}{ll}
\sup _{\nu \in \mathbb{S}^{N-2}}\left|\nabla \varphi(t \nu)-g(\nu) \nu-\nabla_{\mathbb{S}^{N-2}} g(\nu)\right|=O\left(t^{\delta}\right), \quad \text { if } N \geqslant 3, \\
\sup _{\nu \in\{-1,1\}}\left|\varphi^{\prime}(t \nu)-g(\nu) \nu\right|=O\left(t^{\delta}\right), & \text { if } N=2
\end{array} \quad \text { as } t \rightarrow 0^{+},\right. \\
& \left|D^{2} \varphi\left(x^{\prime}\right)\right|=O\left(\left|x^{\prime}\right|^{-1}\right) \quad \text { as }\left|x^{\prime}\right| \rightarrow 0 .
\end{aligned}
$$

As we will show in Lemma 2.1, assumptions (2-5) imply that there exists $C_{0}>0$ such that

$$
\left|\varphi\left(x^{\prime}\right)-\nabla \varphi\left(x^{\prime}\right) \cdot x^{\prime}\right| \leqslant C_{0}\left|x^{\prime}\right|^{1+\delta} \text { for all } x^{\prime} \text { in a neighborhood of } x^{\prime}=0 \text {. }
$$

Furthermore, from (3) it follows that the function $\varphi_{0}: \mathbb{R}^{N-1} \rightarrow \mathbb{R}$,

$$
\varphi_{0}\left(x^{\prime}\right):= \begin{cases}\left|x^{\prime}\right| g\left(\frac{x^{\prime}}{\left|x^{\prime}\right|}\right), & \text { if } x^{\prime} \in \mathbb{R}^{N-1} \backslash\{0\}, \\ 0, & \text { if } x^{\prime}=0,\end{cases}
$$

satisfies

$$
\varphi_{0} \in C^{0}\left(\mathbb{R}^{N-1}\right) \quad \text { and } \quad \varphi_{0} \in C^{1}\left(\mathbb{R}^{N-1} \backslash\{0\}\right) .
$$

Hence the cone in $\mathbb{R}^{N}$ with vertex in 0 defined as

$$
\mathcal{C}:=\left\{\left(x^{\prime}, x_{N}\right) \in \mathbb{R}^{N-1} \times \mathbb{R}: x_{N}>\varphi_{0}\left(x^{\prime}\right)\right\}
$$

is open. In particular,

$$
C=\mathcal{C} \cap \mathbb{S}^{N-1}
$$

is an open connected subset of $\mathbb{S}^{N-1}$.

Let $\Omega$ be an open subset of $\mathbb{R}^{N}$ such that, for some $R>0$,

$$
\Omega \cap B_{R}=\left\{x=\left(x^{\prime}, x_{N}\right) \in B_{R}: x_{N}>\varphi\left(x^{\prime}\right)\right\},
$$

where $B_{R}$ denotes the ball $\left\{x \in \mathbb{R}^{N}:|x|<R\right\}$ in $\mathbb{R}^{N}$ with center at 0 and radius $R$, see figure 1 . 


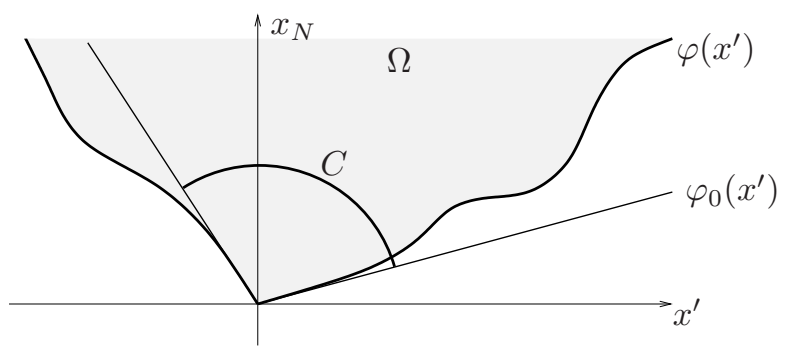

Figure 1. An example of domain $\Omega$.

Let $A: \Omega \rightarrow \mathcal{M}_{N \times N}$ (with $\mathcal{M}_{N \times N}$ denoting the space of $N \times N$ real matrices) satisfying

$$
\left\{\begin{array}{l}
a_{i j}=(A)_{i j} \in W^{1, \infty}(\Omega) \text { for all } i, j=1, \ldots, N, \quad a_{i j}=a_{j i}, \\
\text { there exists } C_{A}>0 \text { such that } A(x) \xi \cdot \xi \geqslant C_{A}|\xi|^{2} \text { for all } \xi \in \mathbb{R}^{N} \text { and } x \in \Omega .
\end{array}\right.
$$

We observe that under assumption (10), the functions $a_{i j}$ are actually Lipschitz continuous functions on $\bar{\Omega}$; moreover, due to symmetry and positive definiteness of $A$, up to some change of variable, it is not restrictive to assume that

$$
A(0)=\operatorname{Id}_{N},
$$

where $\operatorname{Id}_{N}$ denotes the identity $N \times N$ matrix. Let us assume

$$
\begin{aligned}
& \mathbf{b} \in L_{\mathrm{loc}}^{\infty}\left(\Omega, \mathbb{R}^{N}\right), \quad|\mathbf{b}(x)|=O\left(|x|^{-1+\delta}\right) \quad \text { as }|x| \rightarrow 0, \\
& h \in L_{\mathrm{loc}}^{\infty}(\Omega), \quad h(x)=O\left(|x|^{-2+\delta}\right) \quad \text { as }|x| \rightarrow 0 .
\end{aligned}
$$

It is not restrictive to assume that the positive constants $\delta$ 's of formulas (4), (5), (12), and (13) are the same and that $\delta \in(0,1)$. Let $V: \mathbb{S}^{N-1} \rightarrow \mathbb{R}$ such that

$$
\begin{cases}V \equiv 0, & \text { if } N=2, \\ L_{V}:=\sup _{\substack{\theta, \tau \in \mathbb{S}^{N-1} \\ \theta \neq \tau}} \frac{|V(\theta)-V(\tau)|}{|\theta-\tau|}<+\infty \quad \text { and } \quad \Lambda(V)<1, & \text { if } N \geqslant 3,\end{cases}
$$

where, for $N \geqslant 3$,

$$
\Lambda(V):=\sup _{v \in \mathcal{D}^{1,2}(\mathcal{C}) \backslash\{0\}} \frac{\int_{\mathcal{C}}|x|^{-2} V(x /|x|) v^{2}(x) d x}{\int_{\mathcal{C}}|\nabla v(x)|^{2} d x}
$$

and $\mathcal{D}^{1,2}(\mathcal{C})$ denotes the completion of $C_{\mathrm{c}}^{\infty}(\mathcal{C})$ with respect to the norm

$$
\|u\|_{\mathcal{D}^{1,2}(\mathcal{C})}:=\left(\int_{\mathcal{C}}|\nabla u(x)|^{2} d x\right)^{1 / 2} .
$$

Let $f: \Omega \times \mathbb{R} \rightarrow \mathbb{R}$ such that

$$
\begin{aligned}
& f \in C^{0}(\Omega \times \mathbb{R}), \quad F \in C^{1}(\Omega \times \mathbb{R}), \quad s \mapsto f(x, s) \in C^{1}(\mathbb{R}) \text { for a.e. } x \in \Omega,
\end{aligned}
$$

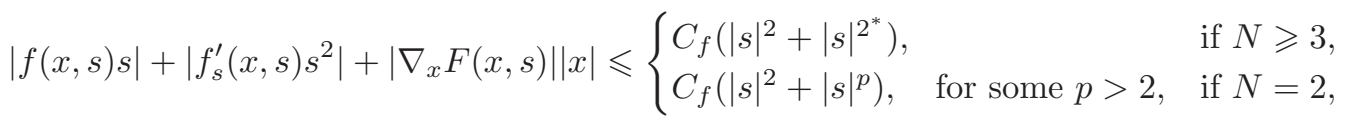

for a.e. $x \in \Omega$ and all $s \in \mathbb{R}$, where $F(x, s)=\int_{0}^{s} f(x, t) d t, 2^{*}=2 N /(N-2)$ is the critical Sobolev exponent, $C_{f}>0$ is a constant independent of $x \in \Omega$ and $s \in \mathbb{R}, \nabla_{x} F$ denotes the gradient of $F$ with respect to the $x$ variable, and $f_{s}^{\prime}(x, s)=\frac{\partial f}{\partial s}(x, s)$.

Let $\mu_{1}(V)$ be the first eigenvalue of the operator $\mathcal{L}_{V}:=-\Delta_{\mathbb{S}^{N-1}}-V$ on the spherical cap $C \subset \mathbb{S}^{N-1}$ under null Dirichlet boundary conditions. By classical spectral theory, the spectrum of the operator $L_{V}$ is discrete and consists in a nondecreasing diverging sequence of eigenvalues

$$
\mu_{1}(V) \leqslant \mu_{2}(V) \leqslant \cdots \leqslant \mu_{k}(V) \leqslant \cdots
$$


with finite multiplicity the first of which admits the variational characterization

$$
\mu_{1}(V)=\min _{\psi \in H_{0}^{1}(C) \backslash\{0\}} \frac{\int_{C}\left[\left|\nabla_{\mathbb{S}^{N-1}} \psi(\theta)\right|^{2}-V(\theta)|\psi(\theta)|^{2}\right] d \sigma(\theta)}{\int_{C}|\psi(\theta)|^{2} d \sigma(\theta)} .
$$

Moreover $\mu_{1}(V)$ is simple and its associated eigenfunctions do not change sign in $C$.

The main result of the present paper provides an evaluation of the behavior at the corner 0 of weak solutions $u \in H^{1}(\Omega)$ to

$$
\begin{cases}-\operatorname{div}(A(x) \nabla u(x))+\mathbf{b}(x) \cdot \nabla u(x)-\frac{V\left(\frac{x}{|x|}\right)}{|x|^{2}} u(x)=h(x) u(x)+f(x, u(x)), & \text { in } \Omega \\ u=0, & \text { on } \partial \Omega \cap B_{R} .\end{cases}
$$

Theorem 1.1. Let $A, \mathbf{b}, f, h, V$ as in assumptions (10-17) and let $\Omega$ satisfying (9) and (2-6). Let $u \in H^{1}(\Omega) \backslash\{0\}$ be a non-trivial weak solution to (19). Then, there exist $k_{0} \in \mathbb{N}, k_{0} \geqslant 1$, and an eigenfunction of the operator $\mathcal{L}_{V}=-\Delta_{\mathbb{S}^{N-1}}-V$ associated to the eigenvalue $\mu_{k_{0}}(V)$ such that $\|\psi\|_{L^{2}\left(\mathbb{S}^{N-1}\right)}=1$ and

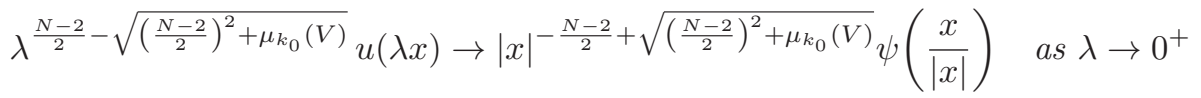

in $H^{1}\left(B_{1}\right)$, in $C_{\text {loc }}^{1, \alpha}\left(\mathcal{C} \cap B_{1}\right)$ and in $C_{\text {loc }}^{0, \alpha}\left(B_{1} \backslash\{0\}\right)$ for any $\alpha \in(0,1)$, with u being trivially extended outside $\Omega$.

As a direct consequence of Theorem 1.1, the following point-wise upper bound holds.

Corollary 1.2. Under the same assumptions as in Theorem 1.1, let $u \in H^{1}(\Omega) \backslash\{0\}$ be a nontrivial weak solution to (19). Then, there exists $k_{0} \in \mathbb{N}, k_{0} \geqslant 1$, such that

$$
u(x)=O\left(|x|^{\left.-\frac{N-2}{2}+\sqrt{\left(\frac{N-2}{2}\right)^{2}+\mu_{k_{0}}(V)}\right)} \quad \text { as }|x| \rightarrow 0^{+} .\right.
$$

A further relevant consequence of our asymptotic analysis is the following unique continuation principle, whose proof follows straightforwardly from Theorem 1.1.

Corollary 1.3. Under the same assumptions as in Theorem 1.1, let $u \in H^{1}(\Omega) \backslash\{0\}$ be a weak solution to (19) such that $u(x)=O\left(|x|^{k}\right)$ as $|x| \rightarrow 0$, for any $k \in \mathbb{N}$. Then $u \equiv 0$ in $\Omega$.

Theorem 1.1 will be proved by introducing an auxiliary equivalent problem obtained as a diffeomorphic deformation of the original problem (19). More precisely, letting $C_{0}$ be as in (7), we define the local diffeomorphism

$$
\Psi: \mathbb{R}^{N} \rightarrow \mathbb{R}^{N}, \quad \Psi(y)=\Psi\left(y^{\prime}, y_{N}\right):=\left(y^{\prime}, y_{N}+2 C_{0}|y|^{1+\delta}\right) .
$$

If $u \in H^{1}(\Omega)$ is a weak solution to (19), then $w=u \circ \Psi$ weakly solves (in the intersection of $\Psi^{-1}(\Omega)$ with a sufficiently small neighborhood of 0 )

$$
-\operatorname{div}(\widetilde{A}(y) \nabla w(y))+\widetilde{\mathbf{b}}(y) \cdot \nabla w(y)-\frac{V\left(\frac{y}{|y|}\right)}{|y|^{2}} w(y)=\widetilde{h}(y) w(y)+\tilde{f}(y, w(y))
$$

where

$$
\begin{aligned}
& \widetilde{A}(y)=|\operatorname{det} \operatorname{Jac} \Psi(y)|(\operatorname{Jac} \Psi(y))^{-1} A(\Psi(y))\left((\operatorname{Jac} \Psi(y))^{T}\right)^{-1}, \\
& \widetilde{\mathbf{b}}(y)=|\operatorname{det} \operatorname{Jac} \Psi(y)| \mathbf{b}(\Psi(y))\left((\operatorname{Jac} \Psi(y))^{T}\right)^{-1}, \\
& \tilde{f}(y, s)=|\operatorname{det} \operatorname{Jac} \Psi(y)| f(\Psi(y), s), \\
& \widetilde{h}(y)=|\operatorname{det} \operatorname{Jac} \Psi(y)| h(\Psi(y))+|\operatorname{det} \operatorname{Jac} \Psi(y)|\left(\frac{V\left(\frac{\Psi(y)}{|\Psi(y)|}\right)}{|\Psi(y)|^{2}}-\frac{V\left(\frac{y}{|y|}\right)}{|y|^{2}}\right) \\
& \quad+(|\operatorname{det} \operatorname{Jac} \Psi(y)|-1) \frac{V\left(\frac{y}{|y|}\right)}{|y|^{2}} .
\end{aligned}
$$

For the auxiliary problem (22) an Almgren monotonicity formula is used to describe the rate and the shape of the singularity of solutions, by relating them to the eigenvalues and the eigenfunctions of the angular operator $\mathcal{L}_{V}$ on the spherical cap $C$. The behavior of solutions of the auxiliary 
problem (22) (and then of the original one (1)) near the corner is indeed classified on the basis of the limit of the following Almgren type frequency function

$$
\mathcal{N}(r)=\frac{r \int_{\Psi^{-1}(\Omega) \cap B_{r}}\left(\widetilde{A} \nabla w \cdot \nabla w+\widetilde{\mathbf{b}} \cdot \nabla w w-\frac{V\left(\frac{y}{y \mid}\right)}{|y|^{2}}|w|^{2}-\widetilde{h} w^{2}-\tilde{f}(y, w) w\right) d y}{\int_{\Psi^{-1}(\Omega) \cap \partial B_{r}} \frac{\widetilde{A}(y) y \cdot y}{|y|^{2}} w^{2}(y) d \sigma(y)},
$$

which is defined for $r>0$ sufficiently small (see (110) and (134)).

Theorem 1.4. Let $A, \mathbf{b}, f, h, V$ as in assumptions (10-17) and let $\Omega$ satisfying (9) and (2-6). Let $u \in H^{1}(\Omega) \backslash\{0\}$ be a non-trivial weak solution to (19) and $w=u \circ \Psi$ with $\Psi$ as in (21). Letting $\mathcal{N}$ as in (27), there exists $k_{0} \in \mathbb{N}, k_{0} \geqslant 1$, such that

$$
\lim _{r \rightarrow 0^{+}} \mathcal{N}(r)=-\frac{N-2}{2}+\sqrt{\left(\frac{N-2}{2}\right)^{2}+\mu_{k_{0}}(V)} .
$$

Furthermore there exists $\psi \in H_{0}^{1}(C) \subset H^{1}\left(\mathbb{S}^{N-1}\right)$ eigenfunction of the operator $\mathcal{L}_{V}=-\Delta_{\mathbb{S}^{N-1}}-V$ associated to the eigenvalue $\mu_{k_{0}}(V)$ such that

$$
\lambda^{\frac{N-2}{2}-\sqrt{\left(\frac{N-2}{2}\right)^{2}+\mu_{k_{0}}(V)}} w(\lambda x) \rightarrow|x|^{-\frac{N-2}{2}+\sqrt{\left(\frac{N-2}{2}\right)^{2}+\mu_{k_{0}}(V)}} \psi\left(\frac{x}{|x|}\right) \quad \text { as } \lambda \rightarrow 0^{+}
$$

in $H^{1}\left(B_{1}\right)$ and in $C_{\text {loc }}^{1, \alpha}\left(\mathcal{C} \cap B_{1}\right)$ for any $\alpha \in(0,1)$.

Furthermore, Theorem 7.6 will provide more precise informations on the limit angular profile $\psi$ : if $m \geqslant 1$ is the multiplicity of the eigenvalue $\mu_{k_{0}}(V)$ and $\left\{\psi_{i}: j_{0} \leqslant i \leqslant j_{0}+m-1\right\}$ is an $L^{2}(C)$ orthonormal basis for the eigenspace associated to $\mu_{k_{0}}(V)$, then the eigenfunction $\psi$ in (28) (which coincides with the one appearing in (20), as clarified in the proof of Theorem 1.1, see section 7) can be written as

$$
\psi(\theta)=\sum_{i=j_{0}}^{j_{0}+m-1} \beta_{i} \psi_{i}(\theta)
$$

where the coefficients $\beta_{i}$ can be represented in terms of the Cauchy's integral type formula (215).

We emphasize that our monotonicity approach allows excluding the presence of logarithmic factors in the leading term of the asymptotic expansion; we refer to [9] for a detailed comparison between the monotonicity approach to asymptotic analysis and the results obtained in earlier literature (see e.g. $[5,6,11,13,14,17,22,23]$ ) by integral representation and Mellin transform methods.

In section 8 we produce an example in dimension $N=2$ of a harmonic function on a domain with a corner of any amplitude and delimited by arcs violating assumptions (4-5), satisfying null Dirichlet boundary conditions but exhibiting dominant logarithmic terms in its asymptotic expansion. Hence assumptions (4-5) are crucial for excluding the presence of logarithms, even under null boundary conditions. Besides the failure of conditions (4-5), other possible reasons of occurring of logarithms in the expansion could be boundary conditions (even if very regular when the amplitude is resonant, see $[14,22]$ ) or lack of linearity with respect to the first derivatives of $u$, see [23].

Notation. We list below some notation used throughout the paper.

- For all $r>0, B_{r}$ denotes the ball $\left\{x \in \mathbb{R}^{N}:|x|<r\right\}$ in $\mathbb{R}^{N}$ with center at 0 and radius $r$.

- $\mathcal{M}_{N \times N}$ denotes the space of $N \times N$ real matrices.

- $\operatorname{Id}_{N}$ denotes the identity $N \times N$ matrix .

- For every vector field $\Psi \in C^{1}\left(\mathbb{R}^{N}, \mathbb{R}^{N}\right)$, Jac $\Psi$ denotes the Jacobian matrix.

\section{An equivalent PRoBlem}

In this section we construct an auxiliary equivalent problem by a diffeomorphic deformation of the domain.

Lemma 2.1. Under assumptions (2-5), there exists $C_{0}>0$ such that (7) holds. 
Proof. From (4) and (5), we can estimate, for $N \geqslant 3$,

$$
\begin{aligned}
& \left|\varphi\left(x^{\prime}\right)-\nabla \varphi\left(x^{\prime}\right) \cdot x^{\prime}\right| \leqslant\left|x^{\prime}\right|\left|\frac{\varphi\left(x^{\prime}\right)}{\left|x^{\prime}\right|}-g\left(\frac{x^{\prime}}{\left|x^{\prime}\right|}\right)\right|+\left|x^{\prime}\right|\left|\left(\nabla \varphi\left(x^{\prime}\right)-g\left(\frac{x^{\prime}}{\left|x^{\prime}\right|}\right) \frac{x^{\prime}}{\left|x^{\prime}\right|}\right) \cdot \frac{x^{\prime}}{\left|x^{\prime}\right|}\right| \\
& =\left|x^{\prime}\right|\left|\frac{\varphi\left(x^{\prime}\right)}{\left|x^{\prime}\right|}-g\left(\frac{x^{\prime}}{\left|x^{\prime}\right|}\right)\right|+\left|x^{\prime}\right|\left|\left(\nabla \varphi\left(x^{\prime}\right)-g\left(\frac{x^{\prime}}{\left|x^{\prime}\right|}\right) \frac{x^{\prime}}{\left|x^{\prime}\right|}-\nabla_{\mathbb{S} N-2} g\left(\frac{x^{\prime}}{\left|x^{\prime}\right|}\right)\right) \cdot \frac{x^{\prime}}{\left|x^{\prime}\right|}\right|=O\left(\left|x^{\prime}\right|^{1+\delta}\right)
\end{aligned}
$$

as $\left|x^{\prime}\right| \rightarrow 0^{+}$thus proving (7). The proof for $N=2$ is similar.

We notice that the function $\Psi$ defined in (21) satisfies $\Psi \in C^{1}\left(\mathbb{R}^{N}, \mathbb{R}^{N}\right)$,

$$
\operatorname{Jac} \Psi\left(y^{\prime}, y_{N}\right)=\left(\begin{array}{ccccc}
1 & 0 & \cdots & 0 & 0 \\
0 & 1 & \cdots & 0 & 0 \\
\vdots & \vdots & & \vdots & \vdots \\
0 & 0 & \cdots & 1 & 0 \\
2 C_{0} \frac{1+\delta}{|y|^{1-\delta}} y_{1} & 2 C_{0} \frac{1+\delta}{|y|^{1-\delta}} y_{2} & \cdots & 2 C_{0} \frac{1+\delta}{|y|^{1-\delta}} y_{N-1} & 1+2 C_{0} \frac{1+\delta}{|y|^{1-\delta}} y_{N}
\end{array}\right)
$$

for all $\left(y^{\prime}, y_{N}\right) \neq 0$, and $\operatorname{Jac} \Psi(0)=\operatorname{Id}_{N}$. Hence there exists a bounded neighborhood $U \subset \mathbb{R}^{N}$ of 0 such that the restriction $\left.\Psi\right|_{U}: U \rightarrow \Psi(U)$ is a $C^{1}$-diffeomorphism. Let us denote as

$$
\widetilde{\Omega}:=\Psi^{-1}(\Omega \cap \Psi(U))
$$

and let us consider the function

$$
\widetilde{\varphi}\left(y^{\prime}\right)=\Psi^{-1}\left(y^{\prime}, \varphi\left(y^{\prime}\right)\right) \cdot e_{N}, \quad e_{N}=(0,0, \cdots, 0,1),
$$

which is well defined in a sufficiently small neighborhood of 0 in $\mathbb{R}^{N-1}$.

Lemma 2.2. There exists $\widetilde{R}>0$ such that

$$
\widetilde{\varphi}\left(y^{\prime}\right)+2 C_{0}\left(\left|y^{\prime}\right|^{2}+\left|\widetilde{\varphi}\left(y^{\prime}\right)\right|^{2}\right)^{\frac{1+\delta}{2}}=\varphi\left(y^{\prime}\right) \quad \text { for all } y^{\prime} \in \mathbb{R}^{N-1},\left|y^{\prime}\right|<\widetilde{R},
$$

and

$$
\widetilde{\Omega} \cap B_{\widetilde{R}}=\left\{\left(y^{\prime}, y_{N}\right) \in B_{\widetilde{R}}: y_{N}>\widetilde{\varphi}\left(y^{\prime}\right)\right\} .
$$

Proof. From the definition of $\widetilde{\varphi}$ we have that

$$
\Psi^{-1}\left(y^{\prime}, \varphi\left(y^{\prime}\right)\right)=\left(y^{\prime}, \widetilde{\varphi}\left(y^{\prime}\right)\right), \quad \text { i.e. } \quad\left(y^{\prime}, \varphi\left(y^{\prime}\right)\right)=\Psi\left(y^{\prime}, \widetilde{\varphi}\left(y^{\prime}\right)\right)
$$

for all $y^{\prime} \in \mathbb{R}^{N-1}$ such that $\left(y^{\prime}, \varphi\left(y^{\prime}\right)\right) \in \Psi(U)$, which implies (31) for some $\widetilde{R}>0$ sufficiently small. To prove (32) we observe that there exists $R_{0}>0$ such that for every fixed $x^{\prime} \in \mathbb{R}^{N-1},\left|x^{\prime}\right|<R_{0}$, the function

$$
t \in\left(-R_{0}, R_{0}\right) \mapsto \Psi^{-1}\left(x^{\prime}, t\right) \cdot e_{N}
$$

is strictly increasing with respect to $t$, since its derivative

$$
\frac{d}{d t}\left(\Psi^{-1}\left(x^{\prime}, t\right) \cdot e_{N}\right)=\frac{1}{1+2 C_{0}(1+\delta)\left|\Psi^{-1}\left(x^{\prime}, t\right)\right|^{-1+\delta} \Psi^{-1}\left(x^{\prime}, t\right) \cdot e_{N}}
$$

is strictly positive provided $R_{0}$ is sufficiently small. In particular, letting $x=\Psi(y)$ in a sufficiently small neighborhood of $0, x_{N}>\varphi\left(x^{\prime}\right)$ if and only if $\Psi^{-1}\left(x^{\prime}, x_{N}\right) \cdot e_{N}>\Psi^{-1}\left(x^{\prime}, \varphi\left(x^{\prime}\right)\right) \cdot e_{N}$ and hence if and only if $y_{N}>\widetilde{\varphi}\left(y^{\prime}\right)$, which, in view of (9) yields the conclusion.

Remark 2.3. From assumption (4) and (31), it follows that

$$
\sup _{\nu \in \mathbb{S}^{N-2}}\left|\frac{\widetilde{\varphi}(t \nu)}{t}-g(\nu)\right|=O\left(t^{\delta}\right) \quad \text { as } t \rightarrow 0^{+}
$$

which implies

$$
\left|\widetilde{\varphi}\left(y^{\prime}\right)-\varphi_{0}\left(y^{\prime}\right)\right|=O\left(\left|y^{\prime}\right|^{1+\delta}\right) \quad \text { as }\left|y^{\prime}\right| \rightarrow 0^{+} .
$$

Furthermore, from assumption (5) and (31), there also holds

$$
\left|\nabla \widetilde{\varphi}\left(y^{\prime}\right)-\nabla \varphi_{0}\left(y^{\prime}\right)\right|=O\left(\left|y^{\prime}\right|^{\delta}\right) \quad \text { as }\left|y^{\prime}\right| \rightarrow 0^{+},
$$

whereas assumption (6) implies that

$$
\left|D^{2} \widetilde{\varphi}\left(y^{\prime}\right)\right|=O\left(\left|y^{\prime}\right|^{-1}\right) \quad \text { as }\left|y^{\prime}\right| \rightarrow 0 .
$$


If $u \in H^{1}(\Omega)$ is a weak solution to (19), then $w=u \circ \Psi \in H^{1}(\widetilde{\Omega})$ is, up to shrinking $\widetilde{R}>0$, a weak solution to

$$
\left\{\begin{array}{lr}
-\operatorname{div}(\widetilde{A}(y) \nabla w(y))+\widetilde{\mathbf{b}}(y) \cdot \nabla w(y)-\frac{V\left(\frac{y}{|y|}\right)}{|y|^{2}} w(y)=\widetilde{h}(y) w(y)+\widetilde{f}(y, w(y)), & \text { in } \widetilde{\Omega}, \\
w=0, & \text { on } \partial \widetilde{\Omega} \cap B_{\widetilde{R}},
\end{array}\right.
$$

where $\widetilde{A}, \widetilde{\mathbf{b}}, \widetilde{h}, \tilde{f}$ are as in $(23-26)$.

Lemma 2.4. Let $A, \mathbf{b}, \Psi, f, h, V$ as in assumptions (10-17), (21), and $\widetilde{A}, \widetilde{\mathbf{b}}, \tilde{f}, \widetilde{h}$ as (23-26). Then

$$
\left\{\begin{array}{l}
\widetilde{A} \in C^{0}\left(\overline{\widetilde{\Omega}}, \mathcal{M}_{N \times N}\right), \quad \widetilde{A} \in W_{\mathrm{loc}}^{1, \infty}\left(\overline{\widetilde{\Omega}} \backslash\{0\}, \mathcal{M}_{N \times N}\right), \quad \widetilde{A}(0)=\operatorname{Id}_{N}, \quad(\widetilde{A})_{i j}=(\widetilde{A})_{j i}, \\
\widetilde{A}(y) \xi \cdot \xi \geqslant C_{\widetilde{A}}|\xi|^{2} \text { for all } \xi \in \mathbb{R}^{N}, y \in \widetilde{\Omega}, \text { and some } C_{\widetilde{A}}>0, \\
\|d \widetilde{A}(y)\|_{\mathcal{L}\left(\mathbb{R}^{N}, \mathcal{M}_{N \times N}\right)}=O\left(|y|^{-1+\delta}\right) \quad \text { and } \quad \widetilde{A}(y)-\operatorname{Id}_{N}=O\left(|y|^{\delta}\right) \quad \text { as }|y| \rightarrow 0,
\end{array}\right.
$$

(39) $\quad \widetilde{\mathbf{b}} \in L_{\mathrm{loc}}^{\infty}\left(\widetilde{\Omega}, \mathbb{R}^{N}\right), \quad|\widetilde{\mathbf{b}}(y)|=O\left(|y|^{-1+\delta}\right) \quad$ as $|y| \rightarrow 0$,

(40) $\tilde{f} \in C^{0}(\widetilde{\Omega} \times \mathbb{R}), \quad \widetilde{F} \in C^{1}(\widetilde{\Omega} \times \mathbb{R}), \quad s \mapsto \tilde{f}(y, s) \in C^{1}(\mathbb{R})$ for a.e. $y \in \widetilde{\Omega}$,

$$
|\tilde{f}(y, s) s|+\left|\tilde{f}_{s}^{\prime}(y, s) s^{2}\right|+\left|\nabla_{y} \widetilde{F}(y, s)\right||y| \leqslant \begin{cases}C_{\tilde{f}}\left(|s|^{2}+|s|^{2^{*}}\right), & \text { if } N \geqslant 3, \\ C_{\tilde{f}}\left(|s|^{2}+|s|^{p}\right), & \text { if } N=2,\end{cases}
$$

(42) $\widetilde{h} \in L_{\mathrm{loc}}^{\infty}(\widetilde{\Omega}), \quad \widetilde{h}(y)=O\left(|y|^{-2+\delta}\right) \quad$ as $|y| \rightarrow 0$,

where $\widetilde{F}(y, s)=\int_{0}^{s} \tilde{f}(y, t) d t=|\operatorname{det} \operatorname{Jac} \Psi(y)| F(\Psi(y), s)$.

Proof. Estimates (38-41) follow from (23-25), (27-28), and assumptions (10-12), (16-17). To prove estimate (42), we first observe that (13) implies $|\operatorname{det} \operatorname{Jac} \Psi(y)| h(\Psi(y))=O\left(|y|^{-2+\delta}\right)$ as $|y| \rightarrow 0$. From (14) and

$$
|\Psi(y)|=|y|\left(1+O\left(|y|^{\delta}\right)\right), \quad \Psi(y)=y+O\left(|y|^{1+\delta}\right) \quad \text { as }|y| \rightarrow 0,
$$

it follows that

$$
\begin{aligned}
& \left|\frac{V\left(\frac{\Psi(y)}{|\Psi(y)|}\right)}{|\Psi(y)|^{2}}-\frac{V\left(\frac{y}{|y|}\right)}{|y|^{2}}\right| \leqslant\left|\frac{V\left(\frac{\Psi(y)}{|\Psi(y)|}\right)-V\left(\frac{y}{|y|}\right)}{|\Psi(y)|^{2}}\right|+\left|V\left(\frac{y}{|y|}\right)\left(\frac{1}{|\Psi(y)|^{2}}-\frac{1}{|y|^{2}}\right)\right| \\
& \leqslant \frac{L_{V}}{|y|^{2}\left(1+O\left(|y|^{\delta}\right)\right)}\left|\frac{\Psi(y)}{|y|\left(1+O\left(|y|^{\delta}\right)\right)}-\frac{y}{|y|}\right|+\|V\|_{L^{\infty}\left(\mathbb{S}^{N-1}\right)}\left|\frac{1}{|y|^{2}\left(1+O\left(|y|^{\delta}\right)\right)}-\frac{1}{|y|^{2}}\right| \\
& =\frac{L_{V}}{|y|^{2}\left(1+O\left(|y|^{\delta}\right)\right)} \frac{\left|\Psi(y)-y+O\left(|y|^{1+\delta}\right)\right|}{|y|\left(1+O\left(|y|^{\delta}\right)\right)}+\|V\|_{L^{\infty}\left(\mathbb{S}^{N-1}\right)} \frac{O\left(|y|^{\delta}\right)}{|y|^{2}\left(1+O\left(|y|^{\delta}\right)\right)}=O\left(|y|^{-2+\delta}\right),
\end{aligned}
$$

which, taking into account that $|\operatorname{det} \operatorname{Jac} \Psi(y)|=1+O\left(|y|^{\delta}\right)$ as $|y| \rightarrow 0$, yields (42).

Lemma 2.5. Let $A$ as in assumptions (10-11) and $\widetilde{A}$ as in (23). Then

$$
(\widetilde{A}(y) y)_{i}= \begin{cases}y_{i}+O\left(|y|^{2}\right), & \text { if } 1 \leqslant i \leqslant N-1, \\ \frac{y_{N}-2 C_{0}(1+\delta)|y|^{-1+\delta}\left|y^{\prime}\right|^{2}}{1+2 C_{0}(1+\delta)|y|^{-1+\delta} y_{N}}+O\left(|y|^{2}\right), & \text { if } i=N,\end{cases}
$$

as $y \rightarrow 0$.

ProOF. The proof follows from (27-28), direct calculations and the estimate

$$
\left|a_{i j}(\Psi(y))-\delta_{i j}\right|=O(|y|) \quad \text { as }|y| \rightarrow 0,
$$

which is a consequence of (10) and (11).

Let us consider the exterior unit normal $\tilde{\nu}$ to $\partial \widetilde{\Omega} \cap B_{\widetilde{R}}$. From (31) and (32), it follows that

$$
\tilde{\nu}\left(y^{\prime}, y_{N}\right)=\frac{\left(\nabla \widetilde{\varphi}\left(y^{\prime}\right),-1\right)}{\sqrt{\left|\nabla \widetilde{\varphi}\left(y^{\prime}\right)\right|^{2}+1}}=\frac{1}{\sqrt{\left|\nabla \widetilde{\varphi}\left(y^{\prime}\right)\right|^{2}+1}}\left(\frac{\nabla \varphi\left(y^{\prime}\right)-2 C_{0}(1+\delta)|y|^{-1+\delta} y^{\prime}}{1+2 C_{0}(1+\delta)|y|^{-1+\delta} y_{N}},-1\right) .
$$


Lemma 2.6. Let $A$ as in assumptions (10-11), $\widetilde{A}$ as in (23), $\widetilde{\Omega}$ as in (29) with $\Omega$ satisfying (9), (2-5), and $\tilde{\nu}$ as in (43). Then $\widetilde{A}(y) y \cdot \tilde{\nu}(y) \geqslant 0$ for all $y \in\left(\partial \widetilde{\Omega} \cap B_{r}\right) \backslash\{0\}$ provided $r$ is sufficiently small.

Proof. Taking into account that $y_{N}=\widetilde{\varphi}\left(y^{\prime}\right)$ and $\left|y^{\prime}\right|^{2}+\left|\widetilde{\varphi}\left(y^{\prime}\right)\right|^{2}=|y|^{2}$ on $\partial \widetilde{\Omega} \cap B_{\widetilde{R}}$, from Lemma $2.5,(43)$, and (31), we deduce that

$$
\begin{aligned}
\left(\sqrt{\left|\nabla \widetilde{\varphi}\left(y^{\prime}\right)\right|^{2}+1}\right) \widetilde{A}(y) y \cdot \tilde{\nu}(y) & =\frac{y^{\prime} \cdot \nabla \varphi\left(y^{\prime}\right)-y_{N}}{1+2 C_{0}(1+\delta)|y|^{-1+\delta} y_{N}}+O\left(|y|^{2}\right) \\
& =\frac{y^{\prime} \cdot \nabla \varphi\left(y^{\prime}\right)-\varphi\left(y^{\prime}\right)+2 C_{0}|y|^{1+\delta}}{1+2 C_{0}(1+\delta)|y|^{-1+\delta} y_{N}}+O\left(|y|^{2}\right) .
\end{aligned}
$$

Hence Lemma 2.1 yields

$$
\left(\sqrt{\left|\nabla \widetilde{\varphi}\left(y^{\prime}\right)\right|^{2}+1}\right) \widetilde{A}(y) y \cdot \tilde{\nu}(y) \geqslant \frac{C_{0}|y|^{1+\delta}}{1+2 C_{0}(1+\delta)|y|^{-1+\delta} y_{N}}+O\left(|y|^{2}\right) \geqslant 0
$$

provided $|y|$ is sufficiently small.

The above lemma ensures that, under assumptions (2-5), (9-11), (29), and (23), up to shrinking $\widetilde{R}>0$ there holds

$$
\begin{gathered}
\widetilde{A}(y) y \cdot \tilde{\nu}(y) \geqslant 0 \quad \text { for all } y \in\left(\partial \widetilde{\Omega} \cap B_{\widetilde{R}}\right) \backslash\{0\} . \\
\text { 3. HARDY TYPE inEQUaLities }(N \geqslant 3)
\end{gathered}
$$

\section{Hardy type inequalities $(N \geqslant 3)$}

Throughout this section we assume $N \geqslant 3$. The following lemma establishes the relation between the values $\Lambda(V)$ defined in (15) and $\mu_{1}(V)$ defined in (18) and the positivity of the quadratic form associated with the principal part of the elliptic operator on the limit domain $\mathcal{C}$ defined in (8).

Lemma 3.1. If $N \geqslant 3$ and $V \in L^{\infty}\left(\mathbb{S}^{N-1}\right)$, then the following conditions are equivalent:

$$
\begin{aligned}
\text { i) } & \inf _{u \in \mathcal{D}^{1,2}(\mathcal{C}) \backslash\{0\}} \frac{\int_{\mathcal{C}}|\nabla v(x)|^{2} d x-\int_{\mathcal{C}} \frac{V(x /|x|)}{|x|^{2}} v^{2}(x) d x}{\int_{\mathcal{C}}|\nabla v(x)|^{2} d x}>0 ; \\
\text { ii) } & \Lambda(V)<1 ; \\
\text { iii) } & \mu_{1}(V)>-\left(\frac{N-2}{2}\right)^{2} .
\end{aligned}
$$

Proof. The equivalence between i) and ii) follows from the definition of $\Lambda(V)$, see (15). The equivalence between i) and iii) can be proved arguing as in [19, Proposition 1.3 and Lemma 1.1].

Let $\widetilde{\Omega}$ be as in (29) with $\Omega$ satisfying (9) and (2-5), and $\widetilde{\varphi}$ be as (30). For every $r \in(0, \tilde{R})$ let us denote

$$
C_{r}=\mathbb{S}^{N-1} \cap\left(\frac{1}{r} \widetilde{\Omega}\right)=\left\{\left(y^{\prime}, y_{N}\right) \in \mathbb{S}^{N-1}: y_{N}>r^{-1} \widetilde{\varphi}\left(r y^{\prime}\right)\right\}
$$

and, for $V \in L^{\infty}\left(\mathbb{S}^{N-1}\right)$, let us consider the first eigenvalue $\mu_{1}(V, r)$ of the operator $-\Delta_{\mathbb{S}^{N-1}}-V$ on the spherical cap $C_{r}$ under null Dirichlet boundary conditions, i.e.

$$
\mu_{1}(V, r)=\min _{\psi \in H_{0}^{1}\left(C_{r}\right) \backslash\{0\}} \frac{\int_{C_{r}}\left[\left|\nabla_{\mathbb{S}^{N-1}} \psi(\theta)\right|^{2}-V(\theta)|\psi(\theta)|^{2}\right] d \sigma(\theta)}{\int_{C_{r}}|\psi(\theta)|^{2} d \sigma(\theta)} .
$$

We also define

$$
\Lambda(V, r)=\max _{\psi \in H_{0}^{1}\left(C_{r}\right) \backslash\{0\}} \frac{\int_{C_{r}} V(\theta)|\psi(\theta)|^{2} d \sigma(\theta)}{\int_{C_{r}}\left[\left|\nabla_{\mathbb{S}^{N-1}} \psi(\theta)\right|^{2}+\left(\frac{N-2}{2}\right)^{2}|\psi(\theta)|^{2}\right] d \sigma(\theta)} .
$$

Lemma 3.2. Let $N \geqslant 3, V \in L^{\infty}\left(\mathbb{S}^{N-1}\right), \mu_{1}(V, r)$ be defined in (46), $\mu_{1}(V)$ in (18), $\Lambda(V, r)$ in (47), and $\Lambda(V)$ in (15). Then

$$
\lim _{r \rightarrow 0^{+}} \mu_{1}(V, r)=\mu_{1}(V)
$$

and

$$
\lim _{r \rightarrow 0^{+}} \Lambda(V, r)=\Lambda(V)
$$


Proof. We first claim that

(50) for every $\psi \in C_{\mathrm{c}}^{\infty}(C)$ there exists $r_{0}>0$ such that $\operatorname{supp} \psi \subseteq C_{r}$ for all $r \in\left(0, r_{0}\right)$.

To prove the claim, let us consider $\psi \in C_{\mathrm{c}}^{\infty}(C)$ and denote $K=\operatorname{supp} \psi$. Since $K$ is compact, we have that

$$
\delta=\min _{\left(y^{\prime}, y_{N}\right) \in K}\left(y_{N}-\varphi_{0}\left(y^{\prime}\right)\right)>0 .
$$

From (33), there exists $r_{0}$ such that

$$
\left|\frac{\widetilde{\varphi}(t \nu)}{t}-g(\nu)\right|<\delta \quad \text { for all } t \in\left(0, r_{0}\right) \text { and for all } \nu \in \mathbb{S}^{N-2} .
$$

Then for all $r \in\left(0, r_{0}\right)$ and $\left(y^{\prime}, y_{N}\right) \in K$ we have that

and hence

$$
\left|\varphi_{0}\left(y^{\prime}\right)-\frac{\widetilde{\varphi}\left(r y^{\prime}\right)}{r}\right|=\left|y^{\prime}\right|\left|\frac{\widetilde{\varphi}\left(r y^{\prime}\right)}{r\left|y^{\prime}\right|}-g\left(\frac{y^{\prime}}{\left|y^{\prime}\right|}\right)\right|<\delta
$$

$$
y_{N}-\frac{\widetilde{\varphi}\left(r y^{\prime}\right)}{r} \geqslant\left(y_{N}-\varphi_{0}\left(y^{\prime}\right)\right)-\left|\varphi_{0}\left(y^{\prime}\right)-\frac{\widetilde{\varphi}\left(r y^{\prime}\right)}{r}\right| \geqslant \delta-\left|\varphi_{0}\left(y^{\prime}\right)-\frac{\widetilde{\varphi}\left(r y^{\prime}\right)}{r}\right|>0
$$

which implies that $K \subseteq C_{r}$ for all $r \in\left(0, r_{0}\right)$, thus proving claim (50).

From (50) it follows that for every $\psi \in C_{\mathrm{c}}^{\infty}(C)$ there exists $r_{0}>0$ such that, for all $r \in\left(0, r_{0}\right)$,

$$
\mu_{1}(V, r) \leqslant \frac{\int_{C_{r}}\left[\left|\nabla_{\mathbb{S}^{N-1}} \psi(\theta)\right|^{2}-V(\theta)|\psi(\theta)|^{2}\right] d \sigma(\theta)}{\int_{C_{r}}|\psi(\theta)|^{2} d \sigma(\theta)}=\frac{\int_{C}\left[\left|\nabla_{\mathbb{S}^{N-1}} \psi(\theta)\right|^{2}-V(\theta)|\psi(\theta)|^{2}\right] d \sigma(\theta)}{\int_{C}|\psi(\theta)|^{2} d \sigma(\theta)} .
$$

Hence

$$
\limsup _{r \rightarrow 0^{+}} \mu_{1}(V, r) \leqslant \frac{\int_{C}\left[\left|\nabla_{\mathbb{S}^{N-1}} \psi(\theta)\right|^{2}-V(\theta)|\psi(\theta)|^{2}\right] d \sigma(\theta)}{\int_{C}|\psi(\theta)|^{2} d \sigma(\theta)}
$$

for all $\psi \in C_{\mathrm{c}}^{\infty}(C)$. By density of $C_{\mathrm{c}}^{\infty}(C)$ in $H_{0}^{1}(C)$, we conclude that

$$
\limsup _{r \rightarrow 0^{+}} \mu_{1}(V, r) \leqslant \mu_{1}(V) \text {. }
$$

To prove (48), it remains to show that

$$
\liminf _{r \rightarrow 0^{+}} \mu_{1}(V, r) \geqslant \mu_{1}(V) .
$$

Arguing by contradiction, let us assume that (51) fails, then there exists $\left\{r_{n}\right\}_{n \in \mathbb{N}} \subset(0, \widetilde{R})$ such that $\lim _{n \rightarrow+\infty} r_{n}=0$ and $\lim _{n \rightarrow+\infty} \mu_{1}\left(V, r_{n}\right)<\mu_{1}(V)$. For all $n$, let $\psi_{n} \in H_{0}^{1}\left(C_{r_{n}}\right)$ such that

$$
\mu_{1}\left(V, r_{n}\right)=\int_{C_{r_{n}}}\left[\left|\nabla_{\mathbb{S}^{N-1}} \psi_{n}(\theta)\right|^{2}-V(\theta)\left|\psi_{n}(\theta)\right|^{2}\right] d \sigma(\theta) \quad \text { and } \quad \int_{C_{r_{n}}}\left|\psi_{n}(\theta)\right|^{2} d \sigma(\theta)=1 \text {. }
$$

Let us identify $\psi_{n}$ with its trivial extension in $\mathbb{S}^{N-1}$ which belongs to $H^{1}\left(\mathbb{S}^{N-1}\right)$. It is easy to verify that $\left\{\psi_{n}\right\}_{n \in \mathbb{N}}$ is bounded in $H^{1}\left(\mathbb{S}^{N-1}\right)$ so that there exists a subsequence $\psi_{n_{k}}$ weakly and a.e. converging to some $\psi$ in $H^{1}\left(\mathbb{S}^{N-1}\right)$. By compactness of the embedding $H^{1}\left(\mathbb{S}^{N-1}\right) \hookrightarrow L^{2}\left(\mathbb{S}^{N-1}\right)$, we have that $\int_{\mathbb{S}^{N-1}} \psi^{2}=1$ and by weakly lower semicontinuity

$$
\begin{aligned}
& \int_{\mathbb{S}^{N-1}}\left[\left|\nabla_{\mathbb{S}^{N-1}} \psi(\theta)\right|^{2}-V(\theta)|\psi(\theta)|^{2}\right] d \sigma(\theta) \\
& \quad \leqslant \liminf _{k \rightarrow \infty} \int_{\mathbb{S}^{N-1}}\left[\left|\nabla_{\mathbb{S}^{N-1}} \psi_{n_{k}}(\theta)\right|^{2}-V(\theta)\left|\psi_{n_{k}}(\theta)\right|^{2}\right] d \sigma(\theta)=\liminf _{k \rightarrow \infty} \mu_{1}\left(V, r_{n_{k}}\right)<\mu_{1}(V) .
\end{aligned}
$$

By a.e. convergence of $\psi_{n_{k}}$ to $\psi$, it is easy to verify that $\psi \in H_{0}^{1}(C)$ thus implying that

$$
\mu_{1}(V) \leqslant \int_{\mathbb{S}^{N-1}}\left[\left|\nabla_{\mathbb{S}^{N-1}} \psi(\theta)\right|^{2}-V(\theta)|\psi(\theta)|^{2}\right] d \sigma(\theta)
$$

giving rise to a contradiction with (52). (48) is thereby proved. The proof of (49) can be derived in similar way after observing that

$$
\Lambda(V)=\max _{\psi \in H_{0}^{1}(C) \backslash\{0\}} \frac{\int_{C} V(\theta)|\psi(\theta)|^{2} d \sigma(\theta)}{\int_{C}\left[\left|\nabla_{\mathbb{S}^{N-1}} \psi(\theta)\right|^{2}+\left(\frac{N-2}{2}\right)^{2}|\psi(\theta)|^{2}\right] d \sigma(\theta)},
$$

see [19, Lemma 1.1]. 
We extend to singular potentials on corner sets the Hardy type inequality with boundary terms proved by Wang and Zhu in [21]. For every $r \in(0, \tilde{R})$ let us denote

$$
\Omega_{r}=\widetilde{\Omega} \cap B_{r}, \quad S_{r}=\left(\partial B_{r}\right) \cap \widetilde{\Omega}, \quad \Gamma_{r}=(\partial \widetilde{\Omega}) \cap B_{r},
$$

so that $\partial \Omega_{r}=\overline{S_{r}} \cup \Gamma_{r}$.

Lemma 3.3. Let $N \geqslant 3$ and $V \in L^{\infty}\left(\mathbb{S}^{N-1}\right)$. For every $r \in(0, \widetilde{R})$ and $v \in H^{1}\left(\Omega_{r}\right)$ such that $v=0$ on $\Gamma_{r}$, the following inequality holds

$$
\begin{aligned}
\int_{\Omega_{r}}\left(|\nabla v(y)|^{2}-\frac{V\left(\frac{y}{|y|}\right)}{|y|^{2}} v^{2}(y)\right) d y+\frac{N-2}{2 r} \int_{S_{r}} v^{2}(y) d \sigma & \\
& \geqslant \int_{\Omega_{r}}\left(\left(\frac{N-2}{2}\right)^{2}+\mu_{1}(V,|y|)\right) \frac{v^{2}(y)}{|y|^{2}} d y .
\end{aligned}
$$

Proof. Let $v \in C_{\mathrm{c}}^{\infty}\left(\widetilde{\Omega} \cap \bar{B}_{r}\right)$ for some $r \in(0, \widetilde{R})$. Passing to polar coordinates and denoting as $\tilde{v}$ the trivial extension of $v$ in $\bar{B}_{r}$, we have that $\tilde{v} \in C^{\infty}\left(\bar{B}_{r}\right)$ and

$$
\begin{aligned}
\int_{\Omega_{r}}\left(|\nabla v(y)|^{2}\right. & \left.-\frac{V\left(\frac{y}{|y|}\right)}{|y|^{2}} v^{2}(y)\right) d y+\frac{N-2}{2 r} \int_{S_{r}} v^{2}(y) d \sigma \\
= & \int_{0}^{r}\left(s^{N-1} \int_{C_{s}}\left|\partial_{s} v(s, \theta)\right|^{2} d \sigma(\theta)\right) d s+\frac{N-2}{2 r} r^{N-1} \int_{C_{r}} v^{2}(r, \theta) d \sigma(\theta) \\
& +\int_{0}^{r}\left(s^{N-3} \int_{C_{s}}\left[\left|\nabla_{\mathbb{S}^{N-1}} v(s, \theta)\right|^{2}-V(\theta) v^{2}(s, \theta)\right] d \sigma(\theta)\right) d s \\
= & \int_{\mathbb{S}^{N-1}}^{r}\left(\int_{0}^{r} s^{N-1}\left|\partial_{s} \tilde{v}(s, \theta)\right|^{2} d s\right) d \sigma(\theta)+\frac{N-2}{2 r} r^{N-1} \int_{\mathbb{S}^{N-1}} \tilde{v}^{2}(r, \theta) d \sigma(\theta) \\
& +\int_{0}^{r}\left(s^{N-3} \int_{C_{s}}\left[\left|\nabla_{\mathbb{S}^{N-1}} v(s, \theta)\right|^{2}-V(\theta) v^{2}(s, \theta)\right] d \sigma(\theta)\right) d s .
\end{aligned}
$$

For all $\theta \in \mathbb{S}^{N-1}$, let $\varphi_{\theta} \in C^{\infty}(0, r)$ be defined by $\varphi_{\theta}(r)=\tilde{v}(r, \theta)$, and $\widetilde{\varphi}_{\theta} \in C^{\infty}\left(B_{r}\right)$ be the radially symmetric function given by $\widetilde{\varphi}_{\theta}(x)=\varphi_{\theta}(|x|)$. We notice that $0 \notin \operatorname{supp} \widetilde{\varphi}_{\theta}$. The Hardy inequality with boundary term proved in [21] yields

$$
\begin{aligned}
\int_{\mathbb{S}^{N-1}} & \left(\int_{0}^{r} s^{N-1}\left|\partial_{s} \tilde{v}(s, \theta)\right|^{2} d s\right) d \sigma(\theta)+\frac{N-2}{2 r} r^{N-1} \int_{\mathbb{S}^{N-1}} \tilde{v}^{2}(r, \theta) d \sigma(\theta) \\
& =\frac{1}{\omega_{N-1}} \int_{\mathbb{S}^{N-1}}\left(\int_{B_{r}}\left|\nabla \widetilde{\varphi}_{\theta}(x)\right|^{2} d x+\frac{N-2}{2 r} \int_{\partial B_{r}}\left|\widetilde{\varphi}_{\theta}(x)\right|^{2} d \sigma\right) d \sigma(\theta) \\
& \geqslant \frac{1}{\omega_{N-1}}\left(\frac{N-2}{2}\right)^{2} \int_{\mathbb{S}^{N-1}}\left(\int_{B_{r}} \frac{\left|\widetilde{\varphi}_{\theta}(x)\right|^{2}}{|x|^{2}} d x\right) d \sigma(\theta) \\
& =\left(\frac{N-2}{2}\right)^{2} \int_{\mathbb{S}^{N-1}}\left(\int_{0}^{r} \frac{s^{N-1}}{s^{2}} \tilde{v}^{2}(s, \theta) d s\right) d \sigma(\theta)=\left(\frac{N-2}{2}\right)^{2} \int_{\Omega_{r}} \frac{v^{2}(x)}{|x|^{2}} d x
\end{aligned}
$$

where $\omega_{N-1}$ denotes the volume of the unit sphere $\mathbb{S}^{N-1}$, i.e. $\omega_{N-1}=\int_{\mathbb{S}^{N-1}} d \sigma(\theta)$. On the other hand, from the definition of $\mu_{1}(V, s)$, see (46), it follows that, for every $s \in(0, r)$,

$$
\int_{C_{s}}\left(\left|\nabla_{\mathbb{S} N-1} v(s, \theta)\right|^{2}-V(\theta) v^{2}(s, \theta)\right) d \sigma(\theta) \geqslant \mu_{1}(s, V) \int_{C_{s}} v^{2}(s, \theta) d \sigma(\theta) .
$$

From (55), (56), and (57), we deduce that

$$
\int_{\Omega_{r}}\left(|\nabla v(y)|^{2}-\frac{V\left(\frac{y}{|y|}\right)}{|y|^{2}} v^{2}(y)\right) d y+\frac{N-2}{2 r} \int_{S_{r}} v^{2}(y) d \sigma \geqslant \int_{\Omega_{r}}\left[\left(\frac{N-2}{2}\right)^{2}+\mu_{1}(|x|, V)\right] \frac{v^{2}(x)}{|x|^{2}} d x
$$

for all $v \in C_{\mathrm{c}}^{\infty}\left(\widetilde{\Omega} \cap \bar{B}_{r}\right)$, which, by density, yields the stated inequality for all $H^{1}\left(\Omega_{r}\right)$-functions vanishing on $\Gamma_{r}$. 
Corollary 3.4. Let $N \geqslant 3$ and $V \in L^{\infty}\left(\mathbb{S}^{N-1}\right)$ such that $\Lambda(V)<1$, where $\Lambda(V)$ is defined in (15). Then, there exist $R_{0} \in(0, \widetilde{R})$ and $C_{N, V}>0$ such that, for every $r \in\left(0, R_{0}\right)$ and $v \in H^{1}\left(\Omega_{r}\right)$ such that $v=0$ on $\Gamma_{r}$, the following inequalities hold

(58) $\int_{\Omega_{r}}\left(|\nabla v(y)|^{2}-\frac{V\left(\frac{y}{|y|}\right)}{|y|^{2}} v^{2}(y)\right) d y+\frac{N-2}{2 r} \int_{S_{r}} v^{2}(y) d \sigma \geqslant \frac{1}{2}\left(\left(\frac{N-2}{2}\right)^{2}+\mu_{1}(V)\right) \int_{\Omega_{r}} \frac{v^{2}(y)}{|y|^{2}} d y$,
(59) $\int_{\Omega_{r}}\left(|\nabla v(y)|^{2}-\frac{V\left(\frac{y}{|y|}\right)}{|y|^{2}} v^{2}(y)\right) d y+\frac{1+\Lambda(V)}{2} \frac{N-2}{2 r} \int_{S_{r}} v^{2}(y) d \sigma \geqslant \frac{1-\Lambda(V)}{2} \int_{\Omega_{r}}|\nabla v(y)|^{2} d y$,

and

$$
\begin{gathered}
\int_{\Omega_{r}}\left(|\nabla v(y)|^{2}-\frac{V\left(\frac{y}{|y|}\right)}{|y|^{2}} v^{2}(y)\right) d y+\frac{N-2}{2 r} \frac{\Lambda(V)+3}{4} \int_{S_{r}} v^{2}(y) d \sigma \\
\geqslant C_{N, V}\left(\int_{\Omega_{r}}\left(|\nabla v(y)|^{2}+\frac{v^{2}(y)}{|y|^{2}}\right) d y+\|v\|_{L^{2^{*}}\left(\Omega_{r}\right)}^{2}\right) .
\end{gathered}
$$

Proof. Inequality (58) follows from Lemmas 3.2 and 3.3. To prove (59) we observe that if $R_{0}$ is sufficiently small, then, by (49) and assumption (14), $\Lambda(V, r)<\frac{\Lambda(V)+1}{2}$ for all $r \in\left(0, R_{0}\right)$. Consequently for all $v \in C_{\mathrm{c}}^{\infty}\left(\widetilde{\Omega} \cap \bar{B}_{r}\right)$ with $r \in\left(0, R_{0}\right)$, from (47) and (56), it follows

$$
\begin{aligned}
& \int_{\Omega_{r}} \frac{V\left(\frac{y}{|y|}\right)}{|y|^{2}} v^{2}(y) d y=\int_{0}^{r} s^{N-3}\left(\int_{C_{s}} V(\theta) v^{2}(s, \theta) d \sigma(\theta)\right) d s \\
& \leqslant \int_{0}^{r} s^{N-3} \Lambda(V, s)\left(\int_{C_{s}}\left[\left|\nabla_{\mathbb{S}^{N-1}} v(s, \theta)\right|^{2}+\left(\frac{N-2}{2}\right)^{2}|v(s, \theta)|^{2}\right] d \sigma(\theta)\right) d s \\
& \leqslant \frac{\Lambda(V)+1}{2} \int_{0}^{r} s^{N-3}\left(\int_{C_{s}}\left[\left|\nabla_{\mathbb{S}^{N-1}} v(s, \theta)\right|^{2}+\left(\frac{N-2}{2}\right)^{2}|v(s, \theta)|^{2}\right] d \sigma(\theta)\right) d s \\
& \leqslant \frac{\Lambda(V)+1}{2} \int_{0}^{r}\left(s^{N-3}\left(\int_{C_{s}}\left|\nabla_{\mathbb{S}^{N-1}} v(s, \theta)\right|^{2} d \sigma(\theta)\right)+s^{N-1}\left(\int_{C_{s}}\left|\partial_{s} \tilde{v}(s, \theta)\right|^{2} d \sigma(\theta)\right)\right) d s \\
&+\frac{\Lambda(V)+1}{2} \frac{N-2}{2 r} r^{N-1} \int_{C_{r}} v^{2}(r, \theta) d \sigma(\theta) \\
&= \frac{\Lambda(V)+1}{2}\left(\int_{\Omega_{r}}|\nabla v(y)|^{2} d y+\frac{N-2}{2 r} \int_{S_{r}} v^{2}(y) d y\right)
\end{aligned}
$$

which yields (59) by density. From summation of (58) and (59) and Sobolev embeddings, it follows that, for every $r \in\left(0, R_{0}\right)$ and $v \in H^{1}\left(\Omega_{r}\right)$ such that $v=0$ on $\Gamma_{r}$,

$$
\begin{aligned}
\int_{\Omega_{r}}\left(|\nabla v(y)|^{2}-\frac{V\left(\frac{y}{|y|}\right)}{|y|^{2}}\right. & \left.v^{2}(y)\right) d y+\frac{N-2}{2 r} \frac{3+\Lambda(V)}{4} \int_{S_{r}} v^{2}(y) d \sigma \\
& \geqslant \frac{\widetilde{S}_{N}}{4} \min \left\{\left(\frac{N-2}{2}\right)^{2}+\mu_{1}(V), 1-\Lambda(V)\right\}\left(\int_{\Omega_{r}}|v(y)|^{2^{*}} d y\right)^{2 / 2^{*}},
\end{aligned}
$$

where $\widetilde{S}_{N}>0$ is the best constant of the Sobolev embedding $H^{1}\left(B_{1}\right) \subset L^{2^{*}}\left(B_{1}\right)$. By summing up (58), (59), (61), we conclude that (60) holds with

$$
C_{N, V}=\frac{\min \left\{1, \widetilde{S}_{N} / 2\right\} \min \left\{\left(\frac{N-2}{2}\right)^{2}+\mu_{1}(V), 1-\Lambda(V)\right\}}{6} .
$$

Repeating the same arguments carried out in this section for the family of domains $\Omega_{r}$, we can prove analogous estimates on the domains $\Omega_{r} \cup \mathcal{C}$.

Corollary 3.5. Under the same assumptions as in Corollary 3.4, there exists $\widetilde{R}_{0}$ such that for every $r \in\left(0, \widetilde{R}_{0}\right)$ and $v \in H^{1}\left(\Omega_{r} \cup \mathcal{C}\right)$ such that $v=0$ on $\partial\left(\Omega_{r} \cup \mathcal{C}\right) \cap B_{r}$, the following inequality 
holds

$$
\begin{gathered}
\int_{\Omega_{r} \cup \mathcal{C}}\left(|\nabla v(y)|^{2}-\frac{V\left(\frac{y}{|y|}\right)}{|y|^{2}} v^{2}(y)\right) d y+\frac{N-2}{2 r} \frac{\Lambda(V)+3}{4} \int_{(\widetilde{\Omega} \cup \mathcal{C}) \cap \partial B_{r}} v^{2}(y) d \sigma \\
\geqslant C_{N, V}\left(\int_{\Omega_{r} \cup \mathcal{C}}\left(|\nabla v(y)|^{2}+\frac{v^{2}(y)}{|y|^{2}}\right) d y+\|v\|_{L^{2^{*}}\left(\Omega_{r} \cup \mathcal{C}\right)}^{2}\right) .
\end{gathered}
$$

\section{A Brezis-Kato type estimate in dimension $N \geqslant 3$}

Throughout this section, we assume $\widetilde{A}, \widetilde{\mathbf{b}}, \widetilde{h}$ as in (23), (24), (26) with $A, \mathbf{b}, \Psi, h, V$ as in assumptions (10-15), (21), and let $\widetilde{\Omega}$ as in (29) with $\Omega$ satisfying (9) and (2-5). We also assume that $W \in L_{\text {loc }}^{1}(\widetilde{\Omega})$ satisfies the form-bounded condition

$$
\sup _{v \in H^{1}(\widetilde{\Omega}) \backslash\{0\}} \frac{\int_{\widetilde{\Omega}}|W(y)| v^{2}(y) d y}{\|v\|_{H^{1}(\widetilde{\Omega})}^{2}}<+\infty,
$$

see [16]. The above condition in particular implies that for every $v \in H^{1}(\widetilde{\Omega}), W v \in H^{-1}(\widetilde{\Omega})$. Let $w \in H^{1}(\widetilde{\Omega}) \backslash\{0\}$ be a weak solution to

$$
\left\{\begin{array}{lr}
-\operatorname{div}(\widetilde{A}(y) \nabla w(y))+\widetilde{\mathbf{b}}(y) \cdot \nabla w(y)-\frac{V\left(\frac{y}{|y|}\right)}{|y|^{2}} w(y)=\widetilde{h}(y) w(y)+W(y) w(y), & \text { in } \widetilde{\Omega}, \\
w=0, & \text { on } \partial \widetilde{\Omega} \cap B_{\widetilde{R}} .
\end{array}\right.
$$

Proposition 4.1. Let $N \geqslant 3$ and let $w$ be a weak solution of (62). If $W_{+} \in L^{N / 2}(\widetilde{\Omega})$, letting

$$
q_{\lim }:= \begin{cases}\frac{2^{*}}{2} \min \left\{\frac{8}{\Lambda(V)+1}-2,2^{*}\right\}, & \text { if } \quad \Lambda(V)>0, \\ \frac{\left(2^{*}\right)^{2}}{2}, & \text { if } \quad \Lambda(V)=0,\end{cases}
$$

then for every $1 \leqslant q<q_{\lim }$ there exists $r_{q}>0$ depending on $q, N, \widetilde{A}, \widetilde{\mathbf{b}}, V, \widetilde{h}$ such that $w \in L^{q}\left(\Omega_{r_{q}}\right)$ with $\Omega_{r_{q}}$ as in (53).

Proof. For any $2<\tau<\frac{2}{2^{*}} q_{\text {lim }}$ define $C(\tau):=\frac{4}{\tau+2}$ and let $\ell_{\tau}>0$ be large enough so that

$$
\left(\int_{\widetilde{\Omega} \cap\left\{W_{+}(y) \geqslant \ell_{\tau}\right\}} W_{+}^{\frac{N}{2}}(y) d y\right)^{\frac{2}{N}}<\frac{S_{N}(2 C(\tau)-\Lambda(V)-1)}{4}
$$

where

$$
S_{N}=\inf _{\phi \in \mathcal{D}^{1,2}\left(\mathbb{R}^{N}\right) \backslash\{0\}} \frac{\int_{\mathbb{R}^{N}}|\nabla \phi(y)|^{2} d y}{\left(\int_{\mathbb{R}^{N}}|\phi(y)|^{2^{*}} d y\right)^{2 / 2^{*}}} .
$$

For any $\phi \in H_{0}^{1}(\widetilde{\Omega})$, by Hölder and Sobolev inequalities and (63), we have

$$
\begin{aligned}
\int_{\widetilde{\Omega}} W(y)|\phi(y)|^{2} d y & \leqslant \ell_{\tau} \int_{\widetilde{\Omega}}|\phi(y)|^{2} d y+\left(\int_{\tilde{\Omega} \cap\left\{W_{+}(y) \geqslant \ell_{\tau}\right\}} W_{+}^{\frac{N}{2}}(y) d y\right)^{\frac{2}{N}}\left(\int_{\widetilde{\Omega}}|\phi(y)|^{2^{*}} d y\right)^{\frac{2}{2^{*}}} \\
& \leqslant \ell_{\tau} \int_{\widetilde{\Omega}}|\phi(y)|^{2} d y+\frac{2 C(\tau)-\Lambda(V)-1}{4} \int_{\widetilde{\Omega}}|\nabla \phi(y)|^{2} d y .
\end{aligned}
$$

Let $r \in(0, \widetilde{R})$ small to chosen later and $\eta \in C_{c}^{\infty}\left(B_{r}\right)$ be such that $\eta \equiv 1$ in $B_{r / 2}$. Let us define $v(y):=\eta(y) w(y) \in H_{0}^{1}\left(\Omega_{r}\right)$. Then $v$ is a $H^{1}\left(\Omega_{r}\right)$-weak solution of the equation

$$
-\operatorname{div}(\widetilde{A}(y) \nabla v(y))+\widetilde{\mathbf{b}}(y) \cdot \nabla v(y)-\frac{V\left(\frac{y}{|y|}\right)}{|y|^{2}} v(y)=\widetilde{h}(y) v(y)+W(y) v(y)+g(y), \quad \text { in } \Omega_{r},
$$

where $g(y)=-\operatorname{div}(\widetilde{A}(y) \nabla \eta(y)) w(y)-2 \widetilde{A}(y) \nabla w(y) \cdot \nabla \eta(y)+(\widetilde{\mathbf{b}}(y) \cdot \nabla \eta(y)) w(y) \in L^{2}\left(\Omega_{r}\right)$. For any $n \in \mathbb{N}, n \geqslant 1$, let us define the function $v^{n}:=\min \{|v|, n\}$. Testing (65) with $\left(v^{n}\right)^{\tau-2} v \in H_{0}^{1}\left(\Omega_{r}\right)$ 
we obtain

(66)

$$
\begin{aligned}
& \int_{\Omega_{r}}\left(v^{n}(y)\right)^{\tau-2} \widetilde{A}(y) \nabla v(y) \cdot \nabla v(y) d y \\
& +(\tau-2) \int_{\Omega_{r}}\left(v^{n}(y)\right)^{\tau-3}|v(y)| \chi_{\{|v(y)|<n\}}(y) \widetilde{A}(y) \nabla v(y) \cdot \nabla v(y) d y-\int_{\Omega_{r}} \frac{V\left(\frac{y}{|y|}\right)}{|y|^{2}}\left(v^{n}(y)\right)^{\tau-2} v^{2}(y) d y \\
& =-\int_{\Omega_{r}}(\widetilde{\mathbf{b}}(y) \cdot \nabla v(y))\left(v^{n}(y)\right)^{\tau-2} v(y) d y+\int_{\Omega_{r}} \widetilde{h}(y)\left(v^{n}(y)\right)^{\tau-2} v^{2}(y) d y \\
& \quad+\int_{\Omega_{r}} W(y)\left(v^{n}(y)\right)^{\tau-2} v^{2}(y) d y+\int_{\Omega_{r}} g(y)\left(v^{n}(y)\right)^{\tau-2} v(y) d y .
\end{aligned}
$$

We observe that the following identities hold true

$$
\left\{\begin{array}{l}
\widetilde{A} \nabla\left(\left(v^{n}\right)^{\frac{\tau}{2}-1} v\right) \cdot \nabla\left(\left(v^{n}\right)^{\frac{\tau}{2}-1} v\right)=\left(v^{n}\right)^{\tau-2} \widetilde{A} \nabla v \cdot \nabla v+\frac{(\tau-2)(\tau+2)}{4}\left(v^{n}\right)^{\tau-2} \chi_{\{|v|<n\}} \widetilde{A} \nabla v \cdot \nabla v \\
\left|\nabla\left(\left(v^{n}\right)^{\frac{\tau}{2}-1} v\right)\right|^{2}=\left(v^{n}\right)^{\tau-2}|\nabla v|^{2}+\frac{(\tau-2)(\tau+2)}{4}\left(v^{n}\right)^{\tau-2} \chi_{\{|v|<n\}}|\nabla v|^{2}
\end{array}\right.
$$

By (39), (67), Hölder and Hardy inequalities, we have

$$
\begin{aligned}
\mid \int_{\Omega_{r}}(\widetilde{\mathbf{b}}(y) \cdot \nabla v(y)) & \left(v^{n}(y)\right)^{\tau-2} v(y) d y\left|\leqslant C_{\widetilde{\mathbf{b}}} \int_{\Omega_{r}}\right| \nabla v(y) \mid \frac{\left(v^{n}(y)\right)^{\tau-2}|v(y)|}{|y|^{1-\delta}} d y \\
& \leqslant C_{\widetilde{\mathbf{b}}} r^{\delta}\left(\int_{\Omega_{r}}\left(v^{n}(y)\right)^{\tau-2}|\nabla v(y)|^{2} d y\right)^{1 / 2}\left(\int_{\Omega_{r}} \frac{\left(\left(v^{n}(y)\right)^{\frac{\tau}{2}-1} v(y)\right)^{2}}{|y|^{2}} d y\right)^{1 / 2} \\
& \leqslant \frac{2 C_{\widetilde{\mathbf{b}}}}{N-2} r^{\delta} \int_{\Omega_{r}}\left|\nabla\left(\left(v^{n}(y)\right)^{\frac{\tau}{2}-1} v(y)\right)\right|^{2} d y
\end{aligned}
$$

for some positive constant $C_{\widetilde{\mathbf{b}}}$ depending only on $\widetilde{\mathbf{b}}$.

Then by (66), (59) applied to the function $\left(v^{n}\right)^{\frac{\tau}{2}-1} v,(64)$ applied to $\phi=\left(v^{n}\right)^{\frac{\tau}{2}-1} v,(68),(38)$, (42) and classical Hardy inequality, we obtain

$$
\begin{gathered}
\leqslant \int_{\Omega_{r}} \frac{V\left(\frac{y}{|y|}\right)}{|y|^{2}}\left(\left(v^{n}(y)\right)^{\frac{\tau}{2}-1} v(y)\right)^{2} d y-\int_{\Omega_{r}}(\widetilde{\mathbf{b}}(y) \cdot \nabla v(y))\left(v^{n}(y)\right)^{\tau-2} v(y) d y+\int_{\Omega_{r}} \widetilde{h}(y)\left(\left(v^{n}(y)\right)^{\frac{\tau}{2}-1} v(y)\right)^{2} d y \\
+\int_{\Omega_{r}} W(y)\left(\left(v^{n}(y)\right)^{\frac{\tau}{2}-1} v(y)\right)^{2} d y+\int_{B_{r}} g(y)\left(v^{n}(y)\right)^{\tau-2} v(y) d y \\
\leqslant\left[\frac{\Lambda(V)+1}{2}+\frac{2 C_{\widetilde{\mathbf{b}}}}{N-2} r^{\delta}+C_{\widetilde{h}}\left(\frac{2}{N-2}\right)^{2} r^{\delta}+\frac{2 C(\tau)-\Lambda(V)-1}{4}\right] \int_{\Omega_{r}}\left|\nabla\left(\left(v^{n}(y)\right)^{\frac{\tau}{2}-1} v(y)\right)\right|^{2} d y \\
+\ell_{\tau} \int_{\Omega_{r}}\left(v^{n}(y)\right)^{\tau-2}(v(y))^{2} d y+\int_{\Omega_{r}}|g(y)|\left(v^{n}(y)\right)^{\tau-2}|v(y)| d y,
\end{gathered}
$$

for some positive constants $K_{\widetilde{A}}$ depending on $\widetilde{A}$ and $C_{\widetilde{h}}$ depending on $\widetilde{h}$.

Arguing as in [9, Proposition 2.3], we can easily estimate

$$
\begin{aligned}
& \int_{\Omega_{r}}|g(y)|\left(v^{n}(y)\right)^{\tau-2}|v(y)| d y \\
& \quad \leqslant \frac{1}{\tau}\|g\|_{L^{2}\left(\Omega_{r}\right)}^{\tau}+\frac{\tau-1}{\tau}\left(\frac{\omega_{N-1}}{N}\right)^{\frac{\tau}{2(\tau-1)}-\frac{2}{2^{*}}} r^{\frac{N \tau}{2(\tau-1)}-N+2} S_{N}^{-1} \int_{\Omega_{r}}\left|\nabla\left(\left(v^{n}(y)\right)^{\frac{\tau}{2}-1} v(y)\right)\right|^{2} d y .
\end{aligned}
$$


Inserting (70) into (69) and using Sobolev embedding, we obtain

$$
\begin{aligned}
S_{N}[ & \frac{2 C(\tau)-\Lambda(V)-1}{4}-\left(C(\tau) K_{\widetilde{A}}+\frac{2 C_{\widetilde{\mathbf{b}}}}{N-2}+C_{h}\left(\frac{2}{N-2}\right)^{2}\right) r^{\delta} \\
& \left.-\frac{\tau-1}{\tau}\left(\frac{\omega_{N-1}}{N}\right)^{\frac{\tau}{2(\tau-1)}-\frac{2}{2^{*}}} r^{\frac{N \tau}{2(\tau-1)}-N+2} S_{N}^{-1}\right]\left(\int_{\Omega_{r}}\left|\left(v^{n}(y)\right)^{\frac{\tau}{2}-1} v(y)\right|^{2^{*}} d y\right)^{2 / 2^{*}} \\
& \leqslant \frac{1}{\tau}\|g\|_{L^{2}\left(\Omega_{r}\right)}^{\tau}+\ell_{\tau} \int_{\Omega_{r}}\left(v^{n}(y)\right)^{\tau-2}(v(y))^{2} d y .
\end{aligned}
$$

Since $\tau<\frac{2}{2^{*}} q_{\lim }$ then $2 C(\tau)-\Lambda(V)-1$ is positive and $\frac{N \tau}{2(\tau-1)}-N+2$ is also positive. Hence we may fix $r$ small enough in such a way that the left hand side of (71) becomes positive. Since $v \in L^{\tau}\left(B_{r}\right)$, letting $n \rightarrow+\infty$, the right hand side of (71) remains bounded and hence by Fatou Lemma we infer that $v \in L^{\frac{2^{*}}{2} \tau}\left(B_{r}\right)$. Since $\eta \equiv 1$ in $B_{r / 2}$ we may conclude that $w \in L^{\frac{2^{*}}{2} \tau}\left(B_{r / 2}\right)$. This completes the proof of the lemma.

\section{THE MONOTONICITY FORMULA}

Let $\widetilde{A}, \widetilde{\mathbf{b}}, \tilde{f}, \widetilde{h}$ be as in (23-26) with $A, \mathbf{b}, \Psi, f, h, V$ as in assumptions (10-17), (21). Let $\widetilde{\Omega}$ be as in (29) with $\Omega$ satisfying (9) and $(2-5)$. Let $w \in H^{1}(\widetilde{\Omega}) \backslash\{0\}$ be a non-trivial weak solution to $(37)$.

For every $r \in(0, \tilde{R})$ let us define

$$
\begin{aligned}
& D(r)=\frac{1}{r^{N-2}} \int_{\Omega_{r}}\left(\widetilde{A} \nabla w \cdot \nabla w+\widetilde{\mathbf{b}} \cdot \nabla w w-\frac{V\left(\frac{y}{|y|}\right)}{|y|^{2}}|w|^{2}-\widetilde{h} w^{2}-\tilde{f}(y, w) w\right) d y, \\
& H(r)=\frac{1}{r^{N-1}} \int_{S_{r}} \mu(y) w^{2}(y) d \sigma(y),
\end{aligned}
$$

where

$$
\mu(y)=\mu\left(y^{\prime}, y_{N}\right)=|y|^{-2} \widetilde{A}(y) y \cdot y .
$$

Lemma 5.1. Let $N \geqslant 2$ and let $\mu$ as in (74) with $\widetilde{A}$ as in (23). Then

$$
\begin{aligned}
& \mu(y)=\frac{1}{1+2 C_{0}(\delta+1) y_{N}|y|^{-1+\delta}}+O(|y|)=1+O\left(|y|^{\delta}\right) \quad \text { as }|y| \rightarrow 0, \\
& \nabla \mu(y)=O\left(|y|^{-1+\delta}\right) \quad \text { as }|y| \rightarrow 0 .
\end{aligned}
$$

Proof. Estimate (75) follows from Lemma 2.5 and direct calculations. Differentiating (74) we obtain

$$
\nabla \mu(y)=-2|y|^{-4}(\widetilde{A}(y) y \cdot y) y+|y|^{-2}(d \widetilde{A}(y) y) y+2|y|^{-2} \widetilde{A}(y) y .
$$

From (75) and (38) we then deduce

$$
\begin{aligned}
\nabla \mu(y) & =-\frac{2|y|^{-2} y}{1+2 C_{0}(\delta+1) y_{N}|y|^{-1+\delta}}+O(1)+|y|^{-2} O\left(|y|^{1+\delta}\right)+2|y|^{-2}\left(y+O\left(|y|^{1+\delta}\right)\right) \\
& =\frac{4 C_{0}(\delta+1) y_{N}|y|^{-3+\delta} y}{1+2 C_{0}(\delta+1) y_{N}|y|^{-1+\delta}}+O\left(|y|^{-1+\delta}\right)=O\left(|y|^{-1+\delta}\right)
\end{aligned}
$$

as $|y| \rightarrow 0$.

Lemma 5.2. Let $N \geqslant 2$ and let $\widetilde{A}, V$ be as in (14), (23) with $A$ as in (10-11). Define the function

$$
\boldsymbol{\beta}(y):=\frac{\widetilde{A}(y) y}{\mu(y)} .
$$


Then we have

$$
\begin{aligned}
& \boldsymbol{\beta}(y)=y+O\left(|y|^{1+\delta}\right)=O(|y|) \text { as }|y| \rightarrow 0 \\
& \operatorname{Jac} \boldsymbol{\beta}(y)=\widetilde{A}(y)+O\left(|y|^{\delta}\right)=\operatorname{Id}_{N}+O\left(|y|^{\delta}\right) \text { as }|y| \rightarrow 0, \\
& \operatorname{div} \boldsymbol{\beta}(y)=N+O\left(|y|^{\delta}\right) \text { as }|y| \rightarrow 0 \\
& \boldsymbol{\beta}(y) \cdot \nabla_{\mathbb{S}^{N-1}} V(y /|y|)=y \cdot \nabla_{\mathbb{S}^{N-1}} V(y /|y|)+O\left(|y|^{1+\delta}\right)=O\left(|y|^{1+\delta}\right) \text { as }|y| \rightarrow 0,
\end{aligned}
$$

Proof. It follows from the definitions of $\boldsymbol{\beta}$ and $\mu$.

From (38-42) we we derive the following lemma.

Lemma 5.3. Let $N \geqslant 2$. Then $H \in W_{\mathrm{loc}}^{1,1}(0, \widetilde{R})$ and

$$
H^{\prime}(r)=\frac{2}{r^{N-1}} \int_{S_{r}} \mu(y) w(y) \frac{\partial w}{\partial \nu}(y) d \sigma(y)+H(r) O\left(r^{-1+\delta}\right) \quad \text { as } r \rightarrow 0^{+}
$$

in a distributional sense and for a.e. $r \in(0, \widetilde{R})$, where $\nu=\nu(y)$ is the unit outer normal vector to $S_{r}$, i.e

$$
\nu(y)=\frac{y}{|y|}
$$

Proof. We notice that, for all $r \in(0, \widetilde{R})$,

$$
H(r)=\int_{C_{r}} \mu(r \theta)|w(r \theta)|^{2} d \sigma
$$

where $C_{r}$ is defined in (45). For every $\phi \in C_{\mathrm{c}}^{\infty}(0, \widetilde{R})$

$$
\begin{aligned}
\int_{0}^{\widetilde{R}} H(t) \phi^{\prime}(t) d t & =\int_{0}^{\widetilde{R}}\left(\int_{C_{t}} \mu(t \theta)|w(t \theta)|^{2} d \sigma\right) \phi^{\prime}(t) d t=\int_{\Omega_{\widetilde{R}}} \frac{\mu(y) w^{2}(y) \nu(y)}{|y|^{N-1}} \cdot \nabla \tilde{\phi}(y) d y \\
& =-\int_{\Omega_{\widetilde{R}}} \operatorname{div}\left(\mu(y) w^{2}(y) \frac{y}{|y|^{N}}\right) \tilde{\phi}(y) d y= \\
& =-\int_{\Omega_{\widetilde{R}}} \frac{w^{2}(y) \nabla \mu(y)+2 w(y) \mu(y) \nabla w(y)}{|y|^{N-1}} \cdot \nu(y) \tilde{\phi}(y) d y \\
& =-\int_{0}^{\widetilde{R}}\left(\int_{C_{t}}\left(2 \mu(t \theta) w(t \theta) \nabla w(t \theta) \cdot \theta+w^{2}(t \theta) \nabla \mu(t \theta) \cdot \theta\right) d \sigma\right) \phi(t) d t
\end{aligned}
$$

where $\tilde{\phi}(y):=\phi(|y|)$. Hence

$$
H^{\prime}(t)=\int_{C_{t}}(2 \mu(t \theta) w(t \theta) \nabla w(t \theta) \cdot \theta) d \sigma+\int_{C_{t}}\left(w^{2}(t \theta) \nabla \mu(t \theta) \cdot \theta\right) d \sigma
$$

in a distributional sense in $(0, \widetilde{R})$. From $w, \frac{\partial w}{\partial \nu} \in L^{2}\left(\Omega_{\widetilde{R}}\right)$ we deduce that $H \in W_{\text {loc }}^{1,1}(0, \widetilde{R})$. Furthermore (83) holds a.e. and can be rewritten as

$$
\begin{aligned}
H^{\prime}(t) & =\frac{2}{t^{N-1}} \int_{S_{t}} \mu(y) w(y) \frac{\partial w}{\partial \nu}(y) d \sigma(y)+\frac{1}{t^{N-1}} \int_{S_{t}} w^{2}(y) \nabla \mu(y) \cdot \nu(y) d \sigma(y) \\
& =\frac{2}{t^{N-1}} \int_{S_{t}} \mu(y) w(y) \frac{\partial w}{\partial \nu}(y) d \sigma(y)+H(t) O\left(t^{-1+\delta}\right)
\end{aligned}
$$

as $t \rightarrow 0^{+}$thus proving (81).

Lemma 5.4. Let $N \geqslant 2$. Let $D$ and $H$ the functions defined in (72-73). Then

$$
\begin{aligned}
H^{\prime}(r) & =\frac{2}{r^{N-1}} \int_{S_{r}}(\widetilde{A} \nabla w \cdot \nu) w d \sigma(y)+H(r) O\left(r^{-1+\delta}\right), \\
H^{\prime}(r) & =\frac{2}{r} D(r)+H(r) O\left(r^{-1+\delta}\right)
\end{aligned}
$$

as $r \rightarrow 0^{+}$. 
Proof. We have that

$$
\int_{S_{r}}(\widetilde{A} \nabla w \cdot \nu) w d \sigma=\int_{S_{r}} \mu w \frac{\partial w}{\partial \nu} d \sigma+\frac{1}{2} \int_{S_{r}} \boldsymbol{\alpha} \cdot \nabla\left(w^{2}\right) d \sigma
$$

where

$$
\boldsymbol{\alpha}(y)=\frac{\mu(y)(\boldsymbol{\beta}(y)-y)}{|y|} .
$$

Since $\boldsymbol{\alpha}(y) \cdot y=0$ and, in view of (76), (77), and (79),

$$
\operatorname{div} \boldsymbol{\alpha}=\left(\frac{\nabla \mu}{|y|}-\mu \frac{y}{|y|^{3}}\right)(\boldsymbol{\beta}-y)+\frac{\mu}{|y|}(\operatorname{div} \boldsymbol{\beta}-N)=O\left(|y|^{-1+\delta}\right) \quad \text { as }|y| \rightarrow 0,
$$

we deduce that

$$
\int_{S_{r}}(\widetilde{A} \nabla w \cdot \nu) w d \sigma=\int_{S_{r}} \mu w \frac{\partial w}{\partial \nu} d \sigma-\frac{1}{2} \int_{S_{r}} \operatorname{div}(\boldsymbol{\alpha}) w^{2} d \sigma=\int_{S_{r}} \mu w \frac{\partial w}{\partial \nu} d \sigma+O\left(r^{-2+N+\delta}\right) H(r)
$$

and hence (84) follows from (81). Multiplying equation (37) by $w$ and integrating on $\Omega_{r}$, from (84) we obtain that

$$
r^{N-2} D(r)=\int_{S_{r}}(\widetilde{A} \nabla w \cdot \nu) w d \sigma=\frac{r^{N-1}}{2} H^{\prime}(r)+O\left(r^{-2+N+\delta}\right) H(r)
$$

as $r \rightarrow 0^{+}$, thus proving (85).

We proceed by distinguishing the cases $N \geqslant 3$ and $N=2$.

5.1. The case $N \geqslant 3$. By (41) and Sobolev embedding, we infer that the function

$$
W(y):= \begin{cases}\frac{\tilde{f}(y, w(y))}{w(y)}, & \text { if } w(y) \neq 0, \\ 0, & \text { if } w(y)=0,\end{cases}
$$

belongs to $L^{N / 2}(\widetilde{\Omega})$ and hence we may apply Proposition 4.1 to the function $w$. Therefore, throughout this section, we may fix

$$
2^{*}<q<q_{\lim }
$$

and $r_{q}$ as in Proposition 4.1 in such a way that $w \in L^{q}\left(\Omega_{r_{q}}\right)$.

Lemma 5.5. There exist $r_{0} \in\left(0, \min \left\{\widetilde{R}, r_{q}\right\}\right)$ and a constant $\bar{C}=\bar{C}(N, V, \widetilde{A}, \widetilde{\mathbf{b}}, \tilde{f}, \widetilde{h}, w)>0$ depending on $N, V, \widetilde{A}, \widetilde{\mathbf{b}}, \tilde{f}, \widetilde{h}, w$ but independent of $r$ such that such that, for all $r \in\left(0, r_{0}\right)$,

(i) $\mu(y)>1 / 2$ for all $y \in B_{r}$,

(ii) $r^{N-2}\left(D(r)+\frac{N-2}{2} H(r)\right) \geqslant \bar{C}\left(\int_{\Omega_{r}}\left(|\nabla w(y)|^{2}+\frac{w^{2}(y)}{|y|^{2}}\right) d y+\|w\|_{L^{2^{*}}\left(\Omega_{r}\right)}^{2}\right)$,

(iii) $H(r)>0$,

where $D$ and $H$ are defined in (72) and (73).

Proof. Estimate $(i)$ near 0 follows from the definition of $\mu$. To prove $(i i)$, we observe that, from (72), (73), (75), and (38-42), it follows that

$$
\begin{aligned}
r^{N-2}(D(r)+ & \left.\frac{N-2}{2} H(r)\right) \geqslant \int_{\Omega_{r}}\left(|\nabla w(y)|^{2}-\frac{V\left(\frac{y}{|y|}\right)}{|y|^{2}} w^{2}(y)\right) d y+\frac{N-2}{2 r}\left(1+O\left(r^{\delta}\right)\right) \int_{S_{r}} w^{2}(y) d \sigma \\
& +O\left(r^{\delta}\right) \int_{\Omega_{r}}\left(|\nabla w(y)|^{2}+\frac{w^{2}(y)}{|y|^{2}}\right) d y-C_{\tilde{f}}\|w\|_{L^{2 *}\left(\Omega_{r}\right)}^{2^{*}-2}\|w\|_{L^{2^{*}}\left(\Omega_{r}\right)}^{2} \\
\geqslant & \int_{\Omega_{r}}\left(|\nabla w(y)|^{2}-\frac{V\left(\frac{y}{|y|}\right)}{|y|^{2}} w^{2}(y)\right) d y+\frac{N-2}{2 r} \frac{\Lambda(V)+3}{4} \int_{S_{r}} w^{2}(y) d \sigma \\
& +\left(O\left(r^{\delta}\right)-C_{\tilde{f}}\|w\|_{L^{2^{*}}\left(\Omega_{r}\right)}^{2^{*}-2}\right)\left(\int_{\Omega_{r}}\left(|\nabla w(y)|^{2}+\frac{w^{2}(y)}{|y|^{2}}\right) d y+\|w\|_{L^{2^{*}}\left(\Omega_{r}\right)}^{2}\right)
\end{aligned}
$$

as $r \rightarrow 0^{+}$, which, together with (60), yields (ii) provided $r$ is sufficiently small.

To prove the positivity of $H$ near 0 , suppose by contradiction that there exists a sequence $r_{n} \rightarrow 0^{+}$such that $H\left(r_{n}\right)=0$. Since $\mu(y)>0$ if $|y|$ is sufficiently small, then $w=0$ a.e. on $S_{r_{n}}$ for 
$n$ sufficiently large and thus $w \in H_{0}^{1}\left(\Omega_{r_{n}}\right)$. Multiplying both sides of (37) by $w$ and using estimate (ii), we obtain, for $n$ sufficiently large,

$$
\begin{aligned}
0 & =\int_{\Omega_{r_{n}}}\left(\widetilde{A} \nabla w \cdot \nabla w+\widetilde{\mathbf{b}} \cdot \nabla w w-\frac{V\left(\frac{y}{|y|}\right)}{|y|^{2}}|w|^{2}-\widetilde{h}|w|^{2}-\tilde{f}(y, w(y)) w\right) d y \\
& \geqslant \bar{C}\left(\int_{\Omega_{r_{n}}}\left(|\nabla w(y)|^{2}+\frac{w^{2}(y)}{|y|^{2}}\right) d y+\|w\|_{L^{2^{*}\left(\Omega_{r_{n}}\right)}}^{2}\right)
\end{aligned}
$$

which implies $w \equiv 0$ in $\Omega_{r_{n}}$ for $n$ large. Applying away from 0 classical unique continuation principles for second order elliptic equations with locally bounded coefficients (see e.g. [24]), we conclude that $w=0$ a.e. in $\widetilde{\Omega}$, a contradiction.

Remark 5.6. If $w \in H^{1}(\widetilde{\Omega})$ is a weak solution to (37), with $\widetilde{A}, \widetilde{\mathbf{b}}, \tilde{f}, \widetilde{h}$ as in $(23-26), A, \mathbf{b}, \Psi, f, h, V$ as in assumptions (10-17), (21), and $\widetilde{\Omega}$ as in (29) with $\Omega$ satisfying (9) and (2), then by classical elliptic regularity theory and a Brezis-Kato type iteration [4], we have that $w \in W_{\text {loc }}^{2, p}(\widetilde{\Omega})$ for all $1 \leqslant p<\infty$. In particular $w \in H_{\mathrm{loc}}^{2}(\widetilde{\Omega}) \cap C_{\mathrm{loc}}^{1, \alpha}(\widetilde{\Omega})$ for all $\alpha \in(0,1)$. Using a local $C^{2}$ parametrization of the boundary away from the origin (see assumption (2)) and classical regularity results for elliptic equations with homogeneous boundary conditions on half-spaces, we can deduce that $w \in C_{\text {loc }}^{1, \alpha}\left(\bar{\Omega}_{\widetilde{R}} \backslash\{0\}\right) \cap H^{2}\left(\Omega_{\widetilde{R}} \backslash \bar{\Omega}_{r}\right)$ for all $r \in(0, \widetilde{R})$.

Proposition 5.7. Let $N \geqslant 3, \widetilde{A}, \widetilde{\mathbf{b}}, \tilde{f}, \widetilde{h}$ as in (23-26) with $A, \mathbf{b}, \Psi, f, h, V$ as in (10-17), (21), and let $\widetilde{\Omega}$ as in (29) with $\Omega$ satisfying (9) and (2-5). If $w \in H^{1}(\widetilde{\Omega}) \backslash\{0\}$ is a weak solution to (3r), then for a.e. $r \in(0, \tilde{R})$

$$
\begin{gathered}
r \int_{S_{r}}(\widetilde{A} \nabla w \cdot \nabla w) d \sigma-2 r \int_{S_{r}} \frac{|\widetilde{A} \nabla w \cdot \nu|^{2}}{\mu} d \sigma-\int_{\Gamma_{r}} \frac{|\nabla w|^{2}}{\mu}(\widetilde{A} \tilde{\nu} \cdot \tilde{\nu})(\widetilde{A} y \cdot \tilde{\nu}) d \sigma \\
=\int_{\Omega_{r}}(\operatorname{div} \boldsymbol{\beta}) \widetilde{A} \nabla w \cdot \nabla w d y-2 \int_{\Omega_{r}}(\operatorname{Jac} \boldsymbol{\beta})(\widetilde{A} \nabla w) \cdot \nabla w d y \\
\quad+\int_{\Omega_{r}}(d \widetilde{A} \nabla w) \nabla w \cdot \boldsymbol{\beta} d y-\int_{\Omega_{r}} 2(\boldsymbol{\beta} \cdot \nabla w)(\widetilde{\mathbf{b}} \cdot \nabla w) d y \\
-\int_{\Omega_{r}} \frac{V(y /|y|) \operatorname{div} \boldsymbol{\beta}-2 V(y /|y|)+|y|^{-1} \boldsymbol{\beta} \cdot \nabla_{\mathbb{S}^{N-1}} V(y /|y|)}{|y|^{2}} w^{2}(y) d y+r \int_{S_{r}} \frac{V(y /|y|)}{|y|^{2}} w^{2} d \sigma \\
\quad+2 \int_{\Omega_{r}}(\boldsymbol{\beta} \cdot \nabla w) \widetilde{h} w d y-2 \int_{\Omega_{r}}\left(\nabla_{y} \widetilde{F}(y, w) \cdot \boldsymbol{\beta}+\widetilde{F}(y, w) \operatorname{div} \boldsymbol{\beta}\right) d y+2 r \int_{S_{r}} \widetilde{F}(y, w) d \sigma
\end{gathered}
$$

where $\boldsymbol{\beta}(y):=\frac{\widetilde{A}(y) y}{\mu(y)}$.

PRoOF. By Remark 5.6, $w \in H_{\text {loc }}^{2}\left(\Omega_{\widetilde{R}}\right) \cap C^{1}\left(\bar{\Omega}_{\widetilde{R}} \backslash\{0\}\right)$ and hence for all $r \in(0, \widetilde{R})$ the following Rellich-Nečas identity

$$
\begin{aligned}
\operatorname{div}((\widetilde{A} \nabla w \cdot \nabla w) \boldsymbol{\beta}-2(\boldsymbol{\beta} \cdot \nabla w) \widetilde{A} \nabla w)=(\operatorname{div} \boldsymbol{\beta}) \widetilde{A} \nabla w \cdot \nabla w-2(\operatorname{Jac} \boldsymbol{\beta})(\widetilde{A} \nabla w) \cdot \nabla w \\
+(d \widetilde{A} \nabla w) \nabla w \cdot \boldsymbol{\beta}-2(\boldsymbol{\beta} \cdot \nabla w)(\widetilde{\mathbf{b}} \cdot \nabla w) \\
\quad+2 \frac{V(y /|y|)}{|y|^{2}}(\boldsymbol{\beta} \cdot \nabla w) w+2(\boldsymbol{\beta} \cdot \nabla w) \widetilde{h} w+2(\boldsymbol{\beta} \cdot \nabla w) \tilde{f}(y, w)
\end{aligned}
$$

is satisfied in a weak sense in $\Omega_{\widetilde{R}} \backslash \bar{\Omega}_{r}$. By (41) and Hardy inequality, we have

$$
\begin{aligned}
\int_{0}^{\widetilde{R}}\left[\int_{S_{s}}\left(|\nabla w(y)|^{2}+\frac{w^{2}(y)}{|y|^{2}}+|\widetilde{F}(y, w(y))|\right) d \sigma\right] d s & \\
& =\int_{\Omega_{\widetilde{R}}}\left(|\nabla w(y)|^{2}+\frac{w^{2}(y)}{|y|^{2}}+|\widetilde{F}(y, w(y))|\right) d y<+\infty
\end{aligned}
$$

and hence there exists a decreasing sequence $\left\{\delta_{n}\right\} \subset(0, \widetilde{R})$ such that $\lim _{n \rightarrow+\infty} \delta_{n}=0$ and

$$
\delta_{n} \int_{S_{\delta_{n}}}\left(|\nabla w(y)|^{2}+\frac{w^{2}(y)}{|y|^{2}}+|\widetilde{F}(y, w(y))|\right) d \sigma \longrightarrow 0 \quad \text { as } n \rightarrow+\infty .
$$


Let $r \in(0, \widetilde{R})$. Integrating (88) in $\Omega_{r} \backslash \Omega_{\delta_{n}}$ and taking into account Remark 5.6, we obtain

$$
\begin{gathered}
\int_{S_{r}}(\widetilde{A} \nabla w \cdot \nabla w) \boldsymbol{\beta} \cdot \nu d \sigma-2 \int_{S_{r}}(\boldsymbol{\beta} \cdot \nabla w) \widetilde{A} \nabla w \cdot \nu d \sigma-\int_{S_{\delta_{n}}}(\widetilde{A} \nabla w \cdot \nabla w) \boldsymbol{\beta} \cdot \nu d \sigma \\
+2 \int_{S_{\delta_{n}}}(\boldsymbol{\beta} \cdot \nabla w) \widetilde{A} \nabla w \cdot \nu d \sigma+\int_{\Gamma_{r} \backslash \Gamma_{\delta_{n}}}(\widetilde{A} \nabla w \cdot \nabla w) \boldsymbol{\beta} \cdot \tilde{\nu} d \sigma-2 \int_{\Gamma_{r} \backslash \Gamma_{\delta_{n}}}(\boldsymbol{\beta} \cdot \nabla w) \widetilde{A} \nabla w \cdot \tilde{\nu} d \sigma \\
=\int_{\Omega_{r} \backslash \Omega_{\delta_{n}}}(\operatorname{div} \boldsymbol{\beta}) \widetilde{A} \nabla w \cdot \nabla w d y-2 \int_{\Omega_{r} \backslash \Omega_{\delta_{n}}}(\operatorname{Jac} \boldsymbol{\beta})(\widetilde{A} \nabla w) \cdot \nabla w d y \\
\quad+\int_{\Omega_{r} \backslash \Omega_{\delta_{n}}}(d \widetilde{A} \nabla w) \nabla w \cdot \boldsymbol{\beta} d y-\int_{\Omega_{r} \backslash \Omega_{\delta_{n}}} 2(\boldsymbol{\beta} \cdot \nabla w)(\widetilde{\mathbf{b}} \cdot \nabla w) d y \\
+2 \int_{\Omega_{r} \backslash \Omega_{\delta_{n}}} \frac{V(y /|y|)}{|y|^{2}}(\boldsymbol{\beta} \cdot \nabla w) w d y+2 \int_{\Omega_{r} \backslash \Omega_{\delta_{n}}}(\boldsymbol{\beta} \cdot \nabla w) \tilde{h} w d y+2 \int_{\Omega_{r} \backslash \Omega_{\delta_{n}}}(\boldsymbol{\beta} \cdot \nabla w) \tilde{f}(y, w) d y
\end{gathered}
$$

with $\nu$ as in (82) and $\tilde{\nu}$ as in (43). Since $\boldsymbol{\beta} \cdot y=|y|^{2}$, integration by parts yields

(91)

$$
\begin{aligned}
\int_{\Omega_{r} \backslash \Omega_{\delta_{n}}} & \frac{V(y /|y|)}{|y|^{2}}(\boldsymbol{\beta} \cdot \nabla w) w d y \\
=-\frac{1}{2} \int_{\Omega_{r} \backslash \Omega_{\delta_{n}}} \frac{V(y /|y|) \operatorname{div} \boldsymbol{\beta}-2 V(y /|y|)+|y|^{-1} \boldsymbol{\beta} \cdot \nabla_{\mathbb{S}^{N-1}} V(y /|y|)}{|y|^{2}} & w^{2}(y) d y \\
& +\frac{r}{2} \int_{S_{r}} \frac{V(y /|y|)}{|y|^{2}} w^{2} d \sigma-\frac{\delta_{n}}{2} \int_{S_{\delta_{n}}} \frac{V(y /|y|)}{|y|^{2}} w^{2} d \sigma
\end{aligned}
$$

and

$$
\begin{aligned}
\int_{\Omega_{r} \backslash \Omega_{\delta_{n}}}(\boldsymbol{\beta} \cdot \nabla w) \tilde{f}(y, w) d y=-\int_{\Omega_{r} \backslash \Omega_{\delta_{n}}}\left(\nabla_{y} \widetilde{F}(y, w) \cdot \boldsymbol{\beta}+\widetilde{F}(y, w) \operatorname{div} \boldsymbol{\beta}\right) d y \\
+r \int_{S_{r}} \widetilde{F}(y, w) d \sigma-\delta_{n} \int_{S_{\delta_{n}}} \widetilde{F}(y, w) d \sigma .
\end{aligned}
$$

By definition of $\boldsymbol{\beta}$

$$
\boldsymbol{\beta} \cdot \nabla w=\frac{r}{\mu} \widetilde{A} \nabla w \cdot \nu \quad \text { on } S_{r} .
$$

Since $w=0$ on $\Gamma_{r}$

$$
\nabla w= \pm|\nabla w| \tilde{\nu} \quad \text { a.e. on } \Gamma_{r}
$$

Taking into account (91-94), (90) becomes

$$
\begin{aligned}
& r \int_{S_{r}}(\widetilde{A} \nabla w\cdot \nabla w) d \sigma-2 r \int_{S_{r}} \frac{|\widetilde{A} \nabla w \cdot \nu|^{2}}{\mu} d \sigma-\delta_{n} \int_{S_{\delta_{n}}}(\widetilde{A} \nabla w \cdot \nabla w) d \sigma \\
&+2 \delta_{n} \int_{S_{\delta_{n}}} \frac{|\widetilde{A} \nabla w \cdot \nu|^{2}}{\mu} d \sigma-\int_{\Gamma_{r} \backslash \Gamma_{\delta_{n}}} \frac{|\nabla w|^{2}}{\mu}(\widetilde{A} \tilde{\nu} \cdot \tilde{\nu})(\widetilde{A} y \cdot \tilde{\nu}) d \sigma \\
&= \int_{\Omega_{r} \backslash \Omega_{\delta_{n}}}(\operatorname{div} \boldsymbol{\beta}) \widetilde{A} \nabla w \cdot \nabla w d y-2 \int_{\Omega_{r} \backslash \Omega_{\delta_{n}}}(\operatorname{Jac} \boldsymbol{\beta})(\widetilde{A} \nabla w) \cdot \nabla w d y \\
&+\int_{\Omega_{r} \backslash \Omega_{\delta_{n}}}(d \widetilde{A} \nabla w) \nabla w \cdot \boldsymbol{\beta} d y-\int_{\Omega_{r} \backslash \Omega_{\delta_{n}}} 2(\boldsymbol{\beta} \cdot \nabla w)(\widetilde{\mathbf{b}} \cdot \nabla w) d y \\
&-\int_{\Omega_{r} \backslash \Omega_{\delta_{n}}} \frac{V(y /|y|) \operatorname{div} \boldsymbol{\beta}-2 V(y /|y|)+|y|^{-1} \boldsymbol{\beta} \cdot \nabla_{\mathbb{S}^{N-1}} V(y /|y|)}{|y|^{2}} w^{2}(y) d y \\
&+r \int_{S_{r}} \frac{V(y /|y|)}{|y|^{2}} w^{2} d \sigma-\delta_{n} \int_{S_{\delta_{n}}} \frac{V(y /|y|)}{|y|^{2}} w^{2} d \sigma+2 \int_{\Omega_{r} \backslash \Omega_{\delta_{n}}}(\boldsymbol{\beta} \cdot \nabla w) \widetilde{h} w d y \\
&\left.-2 \int_{\Omega_{r} \backslash \Omega_{\delta_{n}}}(\nabla / \nabla) \widetilde{F}(y, w) \cdot \boldsymbol{\beta}+\widetilde{F}(y, w) \operatorname{div} \boldsymbol{\beta}\right) d y+2 r \int_{S_{r}} \widetilde{F}(y, w) d \sigma-2 \delta_{n} \int_{S_{\delta_{n}}} \widetilde{F}(y, w) d \sigma .
\end{aligned}
$$


Letting $n \rightarrow \infty$ in (95) and using (89), Lemma 5.2, and (38), we obtain that

$$
\int_{\Gamma_{r}} \frac{|\nabla w|^{2}}{\mu}(\widetilde{A} \tilde{\nu} \cdot \tilde{\nu})(\widetilde{A} y \cdot \tilde{\nu}) d \sigma<+\infty
$$

and (87) holds.

Lemma 5.8. If $q$ is as in (86), the function

$$
g(r):=\frac{r^{\frac{q-2^{*}}{q}} \int_{S_{r}}|w|^{2^{*}} d \sigma}{\left(\int_{\Omega_{r}}|w(y)|^{2 *} d y\right)^{1-\frac{1}{N}}}
$$

is well defined and satisfies

$$
g \in L^{1}\left(0, r_{0}\right) \quad \text { and } \quad g \geqslant 0 \text { a.e. in }\left(0, r_{0}\right) .
$$

Furthermore

$$
\int_{S_{r}}|w|^{2^{*}} d \sigma \leqslant\left(\frac{\omega_{N-1}}{N}\right)^{\frac{q-2^{*}}{q N}}\|w\|_{L^{q}\left(\Omega_{r_{0}}\right)}^{2^{*} / N} \bar{C}^{-1} g(r) r^{N-2}\left(D(r)+\frac{N-2}{2} H(r)\right)
$$

for a.e. $r \in\left(0, r_{0}\right)$ and

$$
\int_{0}^{r} g(s) d s \leqslant N\|w\|_{L^{2^{*}}\left(\Omega_{r_{0}}\right)}^{2^{*} / N} r^{\frac{q-2^{*}}{q}}
$$

for all $r \in\left(0, r_{0}\right)$.

Proof. From Lemma 5.5, $\int_{\Omega_{r}}|w(y)|^{2^{*}} d y>0$ for any $r \in\left(0, r_{0}\right)$ and $g$ is well defined in $\left(0, r_{0}\right)$. Let us denote $\beta=\frac{q-2^{*}}{q}>0$. By a direct calculation, we have that

$$
\begin{aligned}
g(r) & =\frac{r^{\beta} \int_{S_{r}}|w|^{2^{*}} d \sigma}{\left(\int_{\Omega_{r}}|w(y)|^{2^{*}} d y\right)^{1-\frac{1}{N}}} \\
& =N\left[\frac{d}{d r}\left(r^{\beta}\left(\int_{\Omega_{r}}|w(y)|^{2^{*}} d y\right)^{1 / N}\right)-\beta r^{-1+\beta}\left(\int_{\Omega_{r}}|w(y)|^{2^{*}} d y\right)^{1 / N}\right]
\end{aligned}
$$

in the distributional sense and for a.e. $r \in\left(0, r_{0}\right)$. Since

$$
\lim _{r \rightarrow 0^{+}} r^{\beta}\left(\int_{\Omega_{r}}|w(y)|^{2^{*}} d y\right)^{1 / N}=0 \text { and }\left(\int_{\Omega_{r}}|w(y)|^{2^{*}} d y\right)^{1 / N}=O(1)
$$

as $r \rightarrow 0^{+}$, we have that $g \in L^{1}\left(0, r_{0}\right)$. Furthermore, (97) follows from integration of (98).

To prove (96) we observe that, by Hölder inequality, Proposition 4.1, and Lemma 5.5,

$$
\begin{aligned}
& \left(\int_{\Omega_{r}}|w(y)|^{2^{*}} d y\right)^{1-\frac{1}{N}}=\left(\int_{\Omega_{r}}|w(y)|^{2^{*}} d y\right)^{\frac{1}{N}}\left(\int_{\Omega_{r}}|w(y)|^{2^{*}} d y\right)^{\frac{2}{2^{*}}} \\
& \leqslant\left(\frac{\omega_{N-1}}{N}\right)^{\frac{\beta}{N}}\|w\|_{L^{q}\left(\Omega_{r_{0}}\right)}^{\frac{2^{*}}{N}} \bar{C}^{-1} r^{\beta+N-2}\left(D(r)+\frac{N-2}{2} H(r)\right)
\end{aligned}
$$

for all $r \in\left(0, r_{0}\right)$, thus implying (96).

Lemma 5.9. The function $D$ defined in (72) belongs to $W_{\text {loc }}^{1,1}\left(0, r_{0}\right)$ and

(99) $D^{\prime}(r)=B(r)+\frac{1}{r^{N-2}} \int_{S_{r}} \widetilde{\mathbf{b}} \cdot \nabla w w d \sigma+O\left(r^{-1+\delta}+r^{-1+\frac{2\left(q-2^{*}\right)}{q}}+g(r)\right)\left(D(r)+\frac{N-2}{2} H(r)\right)$ as $r \rightarrow 0^{+}$, in a distributional sense and for a.e. $r \in\left(0, r_{0}\right)$, where

$$
B(r):=\frac{2}{r^{N-2}} \int_{S_{r}} \frac{|\widetilde{A} \nabla w \cdot \nu|^{2}}{\mu} d \sigma+\frac{1}{r^{N-1}} \int_{\Gamma_{r}} \frac{|\nabla w|^{2}}{\mu}(\widetilde{A} \tilde{\nu} \cdot \tilde{\nu})(\widetilde{A} y \cdot \tilde{\nu}) d \sigma
$$

and $r_{0}$ is as in Lemma 5.5 . 
Proof. By (87) and Lemmas 2.4 and 5.2, we have that

$$
\begin{aligned}
& \int_{S_{r}}(\widetilde{A} \nabla w \cdot \nabla w) d \sigma-\int_{S_{r}} \frac{V(y /|y|)}{|y|^{2}} w^{2} d \sigma \\
&=2 \int_{S_{r}} \frac{|\widetilde{A} \nabla w \cdot \nu|^{2}}{\mu} d \sigma+\frac{1}{r} \int_{\Gamma_{r}} \frac{|\nabla w|^{2}}{\mu}(\widetilde{A} \tilde{\nu} \cdot \tilde{\nu})(\widetilde{A} y \cdot \tilde{\nu}) d \sigma \\
&+\frac{N-2}{r}\left(\int_{\Omega_{r}} \widetilde{A} \nabla w \cdot \nabla w d y-\int_{\Omega_{r}} \frac{V(y /|y|)}{|y|^{2}} w^{2} d y\right) \\
&-\frac{2}{r} \int_{\Omega_{r}}\left(\nabla_{y} \widetilde{F}(y, w) \cdot y+N \widetilde{F}(y, w)\right) d y+2 \int_{S_{r}} \widetilde{F}(y, w) d \sigma \\
&+O\left(r^{-1+\delta}\right)\left(\int_{\Omega_{r}} \widetilde{A} \nabla w \cdot \nabla w d y+\int_{\Omega_{r}} \frac{w^{2}(y)}{|y|^{2}} d y+\int_{\Omega_{r}}\left(|w|^{2}+|w|^{2^{*}}\right) d y\right) .
\end{aligned}
$$

From (72) and (101) we obtain

$$
\begin{aligned}
D^{\prime}(r)= & -\frac{N-2}{r^{N-1}} \int_{\Omega_{r}}\left(\widetilde{A} \nabla w \cdot \nabla w+\widetilde{\mathbf{b}} \cdot \nabla w w-\frac{V\left(\frac{y}{|y|}\right)}{|y|^{2}}|w|^{2}-\widetilde{h}|w|^{2}-\tilde{f}(y, w(y)) w\right) d y \\
& +\frac{1}{r^{N-2}} \int_{S_{r}}\left(\widetilde{A} \nabla w \cdot \nabla w+\widetilde{\mathbf{b}} \cdot \nabla w w-\frac{V\left(\frac{y}{|y|}\right)}{|y|^{2}}|w|^{2}-\widetilde{h}|w|^{2}-\tilde{f}(y, w(y)) w\right) d \sigma \\
= & B(r)+\frac{1}{r^{N-1}} \int_{\Omega_{r}}\left((N-2) \tilde{f}(y, w(y)) w-2 \nabla_{y} \widetilde{F}(y, w) \cdot y-2 N \tilde{F}(y, w)\right) d y \\
& +\frac{1}{r^{N-2}} \int_{S_{r}}\left(\widetilde{\mathbf{b}} \cdot \nabla w w-\widetilde{h}|w|^{2}-\tilde{f}(y, w(y)) w+2 \widetilde{F}(y, w)\right) d \sigma \\
& +O\left(r^{1-N+\delta}\right)\left(\int_{\Omega_{r}} \widetilde{A} \nabla w \cdot \nabla w d y+\int_{\Omega_{r}} \frac{w^{2}(y)}{|y|^{2}}+\int_{\Omega_{r}}\left(|w|^{2}+|w|^{2^{*}}\right) d y\right) .
\end{aligned}
$$

From (41), Hölder inequality, Proposition 4.1, and Lemma $5.5(i i)$, we have that, for all $r \in\left(0, r_{0}\right)$,

$$
\begin{gathered}
\left|\frac{1}{r^{N-1}} \int_{\Omega_{r}}\left((N-2) \tilde{f}(y, w(y)) w-2 \nabla_{y} \widetilde{F}(y, w) \cdot y-2 N \widetilde{F}(y, w)\right) d y\right| \\
\leqslant \frac{2 N C_{\tilde{f}}}{r^{N-1}} \int_{\Omega_{r}}\left(w^{2}(y)+|w(y)|^{2^{*}}\right) d y \\
\leqslant \frac{2 N C_{\tilde{f}}}{r^{N-1}}\left(\left(\frac{\omega_{N-1}}{N}\right)^{\frac{2}{N}} r^{2}+\|w\|_{L^{2 *}\left(\Omega_{r}\right)}^{2^{*}-2}\right)\left(\int_{\Omega_{r}}|w(y)|^{2^{*}} d y\right)^{2 / 2^{*}} \\
\leqslant \frac{2 N C_{\tilde{f}}}{\bar{C}} r^{-1+\frac{2\left(q-2^{*}\right)}{q}}\left(\left(\frac{\omega_{N-1}}{N}\right)^{\frac{2}{N}} r_{0}^{\frac{22^{*}}{q}}+\left(\frac{\omega_{N-1}}{N}\right)^{\frac{2\left(q-2^{*}\right)}{N q}}\|w\|_{L^{q}\left(\Omega_{r_{0}}\right)}^{2^{*}-2}\right)\left(D(r)+\frac{N-2}{2} H(r)\right) .
\end{gathered}
$$

On the other hand, from (42), Lemma $5.5(i),(73),(41),(96)$, we can estimate

(104) $\frac{1}{r^{N-2}} \int_{S_{r}}\left(\widetilde{h}|w|^{2}+\tilde{f}(y, w(y)) w-2 \widetilde{F}(y, w)\right) d \sigma=O\left(g(r)+r^{-1+\delta}\right)\left(D(r)+\frac{N-2}{2} H(r)\right)$.

In view of (103), (104), and estimate (ii) in Lemma 5.5, (102) yields (99).

Lemma 5.10. Let $D$ and $H$ be the functions defined in (72-73), $r_{0}$ be as in Lemma 5.5, and denote

$$
\Sigma:=\left\{r \in\left(0, r_{0}\right): D^{\prime}(r) H(r) \leqslant H^{\prime}(r) D(r)\right\} .
$$

If $\Sigma \neq \emptyset$ and 0 is a limit point of $\Sigma$, then

$$
D^{\prime}(r)=B(r)+O\left(r^{-1+\delta}+r^{-1+\frac{2\left(q-2^{*}\right)}{q}}+g(r)\right)\left(D(r)+\frac{N-2}{2} H(r)\right)
$$

as $r \rightarrow 0^{+}, r \in \Sigma$. 
Proof. From (87), (77-80), (38-42), Lemma $5.5(i)$, and (73) we have that

$$
\begin{array}{r}
\frac{1}{r^{N-3}} \int_{S_{r}}(\widetilde{A} \nabla w \cdot \nabla w) d \sigma=r B(r)+O\left(r^{-N+2}\right)\left(\int_{\Omega_{r}}\left(|\nabla w(y)|^{2}+\frac{w^{2}(y)}{|y|^{2}}\right) d y+\|w\|_{L^{2^{*}}\left(\Omega_{r}\right)}^{2}\right) \\
+O(1) H(r)+O\left(r^{-N+3}\right) \int_{S_{r}}|w|^{2^{*}} d \sigma
\end{array}
$$

which, in view of Lemma 5.5 and (96), implies

$$
\frac{1}{r^{N-3}} \int_{S_{r}}(\widetilde{A} \nabla w \cdot \nabla w) d \sigma=r B(r)+O(1+r g(r))\left(D(r)+\frac{N-2}{2} H(r)\right)
$$

as $r \rightarrow 0^{+}$. From (39), Schwarz inequality, Lemma $5.5(i),(73),(38)$, and (106), we have that

$$
\begin{aligned}
\frac{1}{r^{N-2}} \int_{S_{r}} \tilde{\mathbf{b}} \cdot \nabla w w d \sigma & =O\left(r^{-1+\delta}\right)\left(\frac{1}{r^{N-3}} \int_{S_{r}}|\nabla w|^{2} d \sigma\right)^{1 / 2} \sqrt{H(r)} \\
& =O\left(r^{-1+\delta}\right)\left(\frac{1}{r^{N-3}} \int_{S_{r}}(\tilde{A} \nabla w \cdot \nabla w) d \sigma\right)^{1 / 2} \sqrt{H(r)} \\
& =O\left(r^{-1+\delta}\right) \sqrt{r B(r) H(r)}+O\left(r^{-1+\delta}+g(r)\right)\left(D(r)+\frac{N-2}{2} H(r)\right)
\end{aligned}
$$

as $r \rightarrow 0^{+}$. From Lemma 5.9 and (107), it follows that

$$
\begin{aligned}
B(r) & =D^{\prime}(r)-\frac{1}{r^{N-2}} \int_{S_{r}} \widetilde{\mathbf{b}} \cdot \nabla w w d \sigma+O\left(r^{-1+\delta}+r^{-1+\frac{2\left(q-2^{*}\right)}{q}}+g(r)\right)\left(D(r)+\frac{N-2}{2} H(r)\right) \\
& =D^{\prime}(r)+O\left(r^{-1+\delta}\right) \sqrt{r B(r) H(r)}+O\left(r^{-1+\delta}+r^{-1+\frac{2\left(q-2^{*}\right)}{q}}+g(r)\right)\left(D(r)+\frac{N-2}{2} H(r)\right) \\
& \leqslant D^{\prime}(r)+\frac{B(r)}{2}+O\left(r^{-1+\delta}+r^{-1+\frac{2\left(q-2^{*}\right)}{q}}+g(r)\right)\left(D(r)+\frac{N-2}{2} H(r)\right)
\end{aligned}
$$

thus yielding

$$
B(r) \leqslant 2 D^{\prime}(r)+O\left(r^{-1+\delta}+r^{-1+\frac{2\left(q-2^{*}\right)}{q}}+g(r)\right)\left(D(r)+\frac{N-2}{2} H(r)\right)
$$

as $r \rightarrow 0^{+}$. From (108), (85), and the fact that $D^{\prime} H \leqslant H^{\prime} D$ a.e. in $\Sigma$, we deduce that, as $r \rightarrow 0^{+}$, $r \in \Sigma$,

$$
\begin{aligned}
r B(r) & H(r) \leqslant 2 r H^{\prime}(r) D(r)+O\left(r^{\delta}+r^{\frac{2\left(q-2^{*}\right)}{q}}+r g(r)\right)\left(D(r)+\frac{N-2}{2} H(r)\right)^{2} \\
& =2 r D(r)\left(\frac{2}{r} D(r)+H(r) O\left(r^{-1+\delta}\right)\right)+O\left(r^{\delta}+r^{\frac{2\left(q-2^{*}\right)}{q}}+r g(r)\right)\left(D(r)+\frac{N-2}{2} H(r)\right)^{2} \\
& =4 D^{2}(r)+O\left(r^{\delta}\right) D(r) H(r)+O\left(r^{\delta}+r^{\frac{2\left(q-2^{*}\right)}{q}}+r g(r)\right)\left(D(r)+\frac{N-2}{2} H(r)\right)^{2} \\
& =O(1+r g(r))\left(D(r)+\frac{N-2}{2} H(r)\right)^{2}
\end{aligned}
$$

which implies

$$
\sqrt{r B(r) H(r)}=O(1+r g(r))\left(D(r)+\frac{N-2}{2} H(r)\right) \quad \text { as } r \rightarrow 0^{+}, r \in \Sigma .
$$

Combining (107) and (109), we obtain

$$
\frac{1}{r^{N-2}} \int_{S_{r}} \widetilde{\mathbf{b}} \cdot \nabla w w d \sigma=O\left(r^{-1+\delta}+g(r)\right)\left(D(r)+\frac{N-2}{2} H(r)\right) \quad \text { as } r \rightarrow 0^{+}, r \in \Sigma
$$

which, together with Lemma 5.9, yields the conclusion.

In view of Lemma 5.5, the Almgren type frequency function

$$
\mathcal{N}(r)=\frac{D(r)}{H(r)}
$$


is well defined in $\left(0, r_{0}\right)$. Furthermore, by Lemmas 5.3 and $5.9, \mathcal{N} \in W_{\mathrm{loc}}^{1,1}\left(0, r_{0}\right)$. The following lemma provides the existence of a finite limit of $\mathcal{N}(r)$ as $r \rightarrow 0^{+}$.

Lemma 5.11. Let $\mathcal{N}:\left(0, r_{0}\right) \rightarrow \mathbb{R}$ be defined in (110). Then the limit

$$
\gamma:=\lim _{r \rightarrow 0^{+}} \mathcal{N}(r)
$$

exists, is finite and

$$
\gamma \geqslant-\frac{N-2}{2}
$$

Proof. By Lemma 5.5,

$$
\mathcal{N}(r)>-\frac{N-2}{2} \text { for all } r \in\left(0, r_{0}\right)
$$

If the set $\Sigma$ defined in (105) is empty or if 0 is not a limit point of $\Sigma$, then $\mathcal{N}^{\prime}(r) \geqslant 0$ in a right neighborhood of 0 and hence $\mathcal{N}$ is nondecreasing near 0 and admits a limit as $r \rightarrow 0^{+}$which is necessarily finite in view of (112). If $\Sigma \neq \emptyset$ and 0 is a limit point of $\Sigma$, then from Lemma 5.10, (85), and (100), we have that

$$
\begin{aligned}
\mathcal{N}^{\prime}(r)= & \frac{D^{\prime}(r) H(r)-H^{\prime}(r) D(r)}{H^{2}(r)} \\
= & \frac{B(r) H(r)}{H^{2}(r)}-\frac{H^{\prime}(r)}{H^{2}(r)}\left(\frac{r}{2} H^{\prime}(r)+H(r) O\left(r^{\delta}\right)\right) \\
& +O\left(r^{-1+\delta}+r^{-1+\frac{2\left(q-2^{*}\right)}{q}}+g(r)\right)\left(\mathcal{N}(r)+\frac{N-2}{2}\right) \\
= & \frac{2 r\left(\int_{S_{r}} \frac{|\widetilde{A} \nabla w \cdot \nu|^{2}}{\mu} d \sigma\right)\left(\int_{S_{r}} \mu w^{2} d \sigma\right)+\left(\int_{S_{r}} \mu w^{2} d \sigma\right)\left(\int_{\Gamma_{r}} \frac{|\nabla w|^{2}(\widetilde{A} \tilde{\nu} \cdot \tilde{\nu})(\widetilde{A} y \cdot \tilde{\nu})}{\mu} d \sigma\right)}{\left(\int_{S_{r}} \mu w^{2} d \sigma\right)^{2}} \\
& -\frac{r}{2} \frac{\left(H^{\prime}(r)\right)^{2}}{H^{2}(r)}+\frac{H^{\prime}(r)}{H(r)} O\left(r^{\delta}\right)+O\left(r^{-1+\delta}+r^{-1+\frac{2\left(q-2^{*}\right)}{q}}+g(r)\right)\left(\mathcal{N}(r)+\frac{N-2}{2}\right)
\end{aligned}
$$

as $r \rightarrow 0^{+}, r \in \Sigma$. In view of (84), there holds

$$
\begin{aligned}
\left(H^{\prime}(r)\right)^{2}=\frac{4}{r^{2 N-2}}\left(\int_{S_{r}}(\widetilde{A} \nabla w\right. & \cdot \nu) w d \sigma(y))^{2} \\
& +H^{2}(r) O\left(r^{-2+2 \delta}\right)+2 H(r) O\left(r^{-1+\delta}\right)\left(H^{\prime}(r)-H(r) O\left(r^{-1+\delta}\right)\right)
\end{aligned}
$$

which yields

$$
\frac{\left(H^{\prime}(r)\right)^{2}}{H^{2}(r)}=\frac{4\left(\int_{S_{r}}(\widetilde{A} \nabla w \cdot \nu) w d \sigma(y)\right)^{2}}{\left(\int_{S_{r}} \mu w^{2} d \sigma\right)^{2}}+O\left(r^{-2+2 \delta}\right)+\frac{H^{\prime}(r)}{H(r)} O\left(r^{-1+\delta}\right) .
$$

Moreover (85) implies

$$
\frac{H^{\prime}(r)}{H(r)}=\frac{2}{r} \mathcal{N}(r)+O\left(r^{-1+\delta}\right) .
$$


From (113), (114), and (115), it follows that

$$
\begin{gathered}
\mathcal{N}^{\prime}(r)=\frac{\int_{\Gamma_{r}} \frac{|\nabla w|^{2}(\tilde{A} \tilde{\nu} \cdot \tilde{\nu})(\widetilde{A} y \cdot \tilde{\nu})}{\mu} d \sigma}{\int_{S_{r}} \mu w^{2} d \sigma} \\
+\frac{2 r\left[\left(\int_{S_{r}} \frac{|\widetilde{A} \nabla w \cdot \nu|^{2}}{\mu} d \sigma\right)\left(\int_{S_{r}} \mu w^{2} d \sigma\right)-\left(\int_{S_{r}}(\widetilde{A} \nabla w \cdot \nu) w d \sigma(y)\right)^{2}\right]}{\left(\int_{S_{r}} \mu w^{2} d \sigma\right)^{2}} \\
+\frac{H^{\prime}(r)}{H(r)} O\left(r^{\delta}\right)+O\left(r^{-1+2 \delta}\right)+O\left(r^{-1+\delta}+r^{-1+\frac{2\left(q-2^{*}\right)}{q}}+g(r)\right)\left(\mathcal{N}(r)+\frac{N-2}{2}\right) \\
=\frac{\int_{\Gamma_{r}} \frac{|\nabla w|^{2}(\tilde{A} \tilde{\nu} \cdot \tilde{\nu})(\widetilde{A} y \cdot \tilde{\nu})}{\mu} d \sigma}{\int_{S_{r}} \mu w^{2} d \sigma}+\frac{2 r\left[\left(\int_{S_{r}} \frac{|\widetilde{A} \nabla w \cdot \nu|^{2}}{\mu} d \sigma\right)\left(\int_{S_{r}} \mu w^{2} d \sigma\right)-\left(\int_{S_{r}}(\widetilde{A} \nabla w \cdot \nu) w d \sigma(y)\right)^{2}\right]}{\left(\int_{S_{r}} \mu w^{2} d \sigma\right)^{2}} \\
\quad+O\left(r^{-1+\delta}\right)+O\left(r^{-1+\delta}+r^{-1+\frac{2\left(q-2^{*}\right)}{q}}+g(r)\right)\left(\mathcal{N}(r)+\frac{N-2}{2}\right)
\end{gathered}
$$

as $r \rightarrow 0^{+}, r \in \Sigma$. From (116), Lemma 2.6, and Schwarz inequality, it follows that

$$
\mathcal{N}^{\prime}(r) \geqslant O\left(r^{-1+\delta}+r^{-1+\frac{2\left(q-2^{*}\right)}{q}}+g(r)\right)\left(\mathcal{N}(r)+\frac{N}{2}\right)
$$

as $r \rightarrow 0^{+}, r \in \Sigma$. Since $\mathcal{N}^{\prime}(r) \geqslant 0$ a.e. in $\left(0, r_{0}\right) \backslash \Sigma$, the above inequality is trivially satisfied as $r \rightarrow 0^{+}, r \in\left(0, r_{0}\right) \backslash \Sigma$. Hence there exists some $r_{1} \in\left(0, r_{0}\right)$ and $c_{1}>0$ such that

$$
\left(\mathcal{N}+\frac{N}{2}\right)^{\prime}(r) \geqslant-c_{1}\left(\mathcal{N}(r)+\frac{N}{2}\right)\left(r^{-1+\delta}+r^{-1+\frac{2\left(q-2^{*}\right)}{q}}+g(r)\right)
$$

for a.e. $r \in\left(0, r_{1}\right)$. After integration over $\left(r, r_{1}\right)$ it follows that

$$
\mathcal{N}(r) \leqslant-\frac{N}{2}+\left(\mathcal{N}\left(r_{1}\right)+\frac{N}{2}\right) \exp \left(c_{1}\left(\frac{r_{1}^{\delta}}{\delta}+\frac{q}{2\left(q-2^{*}\right)} r_{1}^{\frac{2\left(q-2^{*}\right)}{q}}+\int_{0}^{r_{1}} g(s) d s\right)\right)
$$

for any $r \in\left(0, r_{1}\right)$, thus proving that there exists $c_{2}>0$ such that

$$
\mathcal{N}(r) \leqslant c_{2} \quad \text { for all } r \in\left(0, r_{1}\right) .
$$

Estimates (117), (118), and the fact that $r \mapsto r^{-1+\delta}+r^{-1+\frac{2\left(q-2^{*}\right)}{q}}+g(r) \in L^{1}\left(0, r_{1}\right)$ imply that $\mathcal{N}^{\prime}$ is the sum of a nonnegative function and of a $L^{1}$-function on $\left(0, r_{1}\right)$. Therefore

$$
\mathcal{N}(r)=\mathcal{N}\left(r_{1}\right)-\int_{r}^{r_{1}} \mathcal{N}^{\prime}(s) d s
$$

admits a limit as $r \rightarrow 0^{+}$which is necessarily finite in view of (118) and (112).

Lemma 5.12. There exists $r_{1} \in\left(0, r_{0}\right)$ and $K_{1}>0$ such that

$$
H(r) \leqslant K_{1} r^{2 \gamma} \text { for all } r \in\left(0, r_{1}\right)
$$

and

$$
H(2 r) \leqslant K_{1} H(r) \quad \text { for all } r \in\left(0, r_{1} / 2\right) .
$$

Furthermore, for any $\sigma>0$ there exists a constant $K_{2}(\sigma)>0$ depending on $\sigma$ such that

$$
H(r) \geqslant K_{2}(\sigma) r^{2 \gamma+\sigma} \quad \text { for all } r \in\left(0, r_{1}\right) .
$$

Proof. By (112), (118), and Lemma 5.11, there exists $r_{1} \in\left(0, r_{0}\right)$ such that $\mathcal{N}$ is bounded in $\left(0, r_{1}\right)$ and $\mathcal{N}^{\prime} \in L^{1}\left(0, r_{1}\right)$. Then from (97) and (117) it follows that

$$
\mathcal{N}(r)-\gamma=\int_{0}^{r} \mathcal{N}^{\prime}(s) d s \geqslant-c_{3} r^{\tilde{\delta}}
$$

for some constant $c_{3}>0$ and all $r \in\left(0, r_{1}\right)$, where

$$
\tilde{\delta}=\min \left\{\delta, \frac{q-2^{*}}{q}\right\} .
$$


Therefore by (85) and (122) we deduce that, for $r \in\left(0, r_{1}\right)$,

$$
\frac{H^{\prime}(r)}{H(r)}=\frac{2 \mathcal{N}(r)}{r}+O\left(r^{-1+\delta}\right) \geqslant \frac{2 \gamma}{r}-2 c_{3} r^{-1+\tilde{\delta}}+O\left(r^{-1+\delta}\right) \quad \text { as } r \rightarrow 0^{+},
$$

which, after integration over the interval $\left(r, r_{1}\right)$ and up to shrinking $r_{1}$, yields (119). On the other hand, from boundedness of $\mathcal{N}$ in $\left(0, r_{1}\right)$, we have that

$$
\frac{H^{\prime}(r)}{H(r)}=\frac{2 \mathcal{N}(r)}{r}+O\left(r^{-1+\delta}\right) \leqslant \frac{\text { const }}{r},
$$

which, for all $r \in\left(0, r_{1} / 2\right)$, after integration over the interval $(r, 2 r)$ yields

$$
\log \frac{H(2 r)}{H(r)} \leqslant \text { const } \log 2
$$

thus proving (120).

Let us prove (121). Since $\gamma=\lim _{r \rightarrow 0^{+}} \mathcal{N}(r)$ and $\frac{H^{\prime}(r)}{H(r)}-\frac{2 \mathcal{N}(r)}{r}=O\left(r^{-1+\delta}\right)$, for any $\sigma>0$ there exists $r_{\sigma}>0$ such that $\mathcal{N}(r)<\gamma+\sigma / 4$ and $\frac{H^{\prime}(r)}{H(r)}-\frac{2 \mathcal{N}(r)}{r} \leqslant \frac{\sigma}{2 r}$ for any $r \in\left(0, r_{\sigma}\right)$ and hence

$$
\frac{H^{\prime}(r)}{H(r)}<\frac{2 \gamma+\sigma}{r} \text { for all } r \in\left(0, r_{\sigma}\right) .
$$

Integrating over the interval $\left(r, r_{\sigma}\right)$ and by continuity of $H$ outside 0 , we obtain (121) for some constant $K_{2}(\sigma)$ depending on $\sigma$.

5.2. The case $N=2$. The two-dimensional version of Lemma 5.5 we are going to prove in Lemma 5.14 requires the following Sobolev type inequality with boundary terms.

Proposition 5.13. Let $N \geqslant 2$ and let $p \in[1, \infty)$ with $p \leqslant 2^{*}=2 N /(N-2)$ if $N \geqslant 3$. Then there exists a constant $C(N, p)>0$ depending only on $N$ and $p$ such that for all $r>0$

$$
\|v\|_{L^{p}\left(B_{r}\right)}^{2} \leqslant C(N, p) r^{\frac{2 N}{p}+2-N}\left(\int_{B_{r}}|\nabla v(x)|^{2} d x+\frac{1}{r} \int_{\partial B_{r}} v^{2}(x) d \sigma\right) \quad \text { for all } v \in H^{1}\left(B_{r}\right)
$$

Proof. The proof in the case $r=1$ follows from the classical Sobolev inequality and the fact that the square root of the right hand side of (124) is a norm equivalent to the standard norm of $H^{1}\left(B_{1}\right)$. The proof in the case of a general $r>0$ follows by scaling.

In the rest of this subsection, we assume $N=2$.

Lemma 5.14. Let $N=2$ and let $p>2$ as in (17). Then for every $\varepsilon>0$ there exist $\tilde{r}_{\varepsilon} \in(0, \widetilde{R})$ and a constant $\bar{C}_{\varepsilon}=\bar{C}_{\varepsilon}(\varepsilon, p, \widetilde{A}, \widetilde{\mathbf{b}}, \tilde{f}, \widetilde{h}, w)>0$ depending on $\varepsilon, p, \widetilde{A}, \widetilde{\mathbf{b}}, \tilde{f}, \widetilde{h}, w$ such that, for all $r \in\left(0, \tilde{r}_{\varepsilon}\right)$,

$$
\begin{aligned}
\text { (i) } & \mu(y)>1 / 2 \quad \text { for all } y \in B_{r}, \\
\text { (ii) } & D(r)+\varepsilon H(r) \geqslant \bar{C}_{\varepsilon}\left(\int_{\Omega_{r}}|\nabla w(y)|^{2} d y+\frac{1}{r} \int_{S_{r}} w^{2} d \sigma+\|w\|_{L^{p}\left(\Omega_{r}\right)}^{2}\right), \\
\text { (iii) } & H(r)>0,
\end{aligned}
$$

where $D$ and $H$ are defined in (72) and (73).

Proof. The positivity of $\mu$ near 0 follows from its definition. By (42), Hölder inequality, and Proposition 5.13, we have

$$
\begin{gathered}
\left|\int_{\Omega_{r}} \widetilde{h}(y) w^{2}(y) d y\right| \leqslant O(1) \int_{\Omega_{r}}|y|^{-2+\delta} w^{2}(y) d y \\
\leqslant O(1)\left(\int_{\Omega_{r}}|y|^{-\frac{4-\delta}{2}} d y\right)^{\frac{2(2-\delta)}{4-\delta}}\|w\|_{L^{\frac{2(4-\delta)}{\delta}\left(\Omega_{r}\right)}}^{2}=O\left(r^{\delta}\right)\left(\int_{\Omega_{r}}|\nabla w(y)|^{2} d y+\frac{1}{r} \int_{S_{r}} w^{2} d \sigma\right)
\end{gathered}
$$


as $r \rightarrow 0^{+}$. Similarly by (39), Hölder inequality, and (125) we also have

$$
\begin{aligned}
\left|\int_{\Omega_{r}} \widetilde{\mathbf{b}}(y) \cdot \nabla w(y) w(y) d y\right| & \leqslant\left(\int_{\Omega_{r}}|\nabla w(y)|^{2} d y\right)^{1 / 2}\left(\int_{\Omega_{r}}|y|^{-2+2 \delta} w^{2}(y) d y\right)^{1 / 2} \\
& =O\left(r^{\delta}\right)\left(\int_{\Omega_{r}}|\nabla w(y)|^{2} d y+\frac{1}{r} \int_{S_{r}} w^{2} d \sigma\right) .
\end{aligned}
$$

as $r \rightarrow 0^{+}$. Finally by (41) and Proposition 5.13 we have

$$
\begin{aligned}
\left|\int_{\Omega_{r}} \tilde{f}(y, w(y)) w(y) d y\right| & \leqslant C_{\tilde{f}} \int_{\Omega_{r}}\left(w^{2}(y)+|w(y)|^{p}\right) d y \\
& \leqslant O\left(r^{\frac{4}{p}}\right)\left(\int_{\Omega_{r}}|\nabla w(y)|^{2} d y+\frac{1}{r} \int_{S_{r}} w^{2} d \sigma\right)
\end{aligned}
$$

as $r \rightarrow 0^{+}$. Inequality (ii) follows from (125-127). Inequality (ii) implies (iii) by proceeding like in the proof of Lemma 5.5.

In view of the previous lemma, we can define the Almgren type frequency function $\mathcal{N}$ as in the previous subsection, see (110). We now sketch the proof of the existence of a finite limit of $\mathcal{N}$ as $r \rightarrow 0^{+}$in dimension $N=2$. To this aim, we first notice that, under assumption (14), the Pohozaev-type identity (87) proved for $N \geqslant 3$ admits the following extension to the twodimensional case.

Proposition 5.15. Let $N=2$ and let $\widetilde{A}, \widetilde{\mathbf{b}}, \tilde{f}, \widetilde{h}$ be as in (23-26) with $A, \mathbf{b}, \Psi, f, h, V$ as in assumptions (10-17), (21), and let $\widetilde{\Omega}$ as in (29) with $\Omega$ satisfying (9) and (2-5). If $w \in H^{1}(\widetilde{\Omega}) \backslash\{0\}$ is a weak solution to (37), then for a.e. $r \in(0, \tilde{R})$

$$
\begin{aligned}
& r \int_{S_{r}}(\widetilde{A} \nabla w \cdot \nabla w) d \sigma-2 r \int_{S_{r}} \frac{|\widetilde{A} \nabla w \cdot \nu|^{2}}{\mu} d \sigma-\int_{\Gamma_{r}} \frac{|\nabla w|^{2}}{\mu}(\widetilde{A} \tilde{\nu} \cdot \tilde{\nu})(\widetilde{A} y \cdot \tilde{\nu}) d \sigma \\
& =\int_{\Omega_{r}}(\operatorname{div} \boldsymbol{\beta}) \widetilde{A} \nabla w \cdot \nabla w d y-2 \int_{\Omega_{r}}(\operatorname{Jac} \boldsymbol{\beta})(\widetilde{A} \nabla w) \cdot \nabla w d y \\
& \quad+\int_{\Omega_{r}}(d \widetilde{A} \nabla w) \nabla w \cdot \boldsymbol{\beta} d y-\int_{\Omega_{r}} 2(\boldsymbol{\beta} \cdot \nabla w)(\widetilde{\mathbf{b}} \cdot \nabla w) d y \\
& \quad+2 \int_{\Omega_{r}}(\boldsymbol{\beta} \cdot \nabla w) \widetilde{h} w d y-2 \int_{\Omega_{r}}\left(\nabla_{y} \widetilde{F}(y, w) \cdot \boldsymbol{\beta}+\widetilde{F}(y, w) \operatorname{div} \boldsymbol{\beta}\right) d y+2 r \int_{S_{r}} \widetilde{F}(y, w) d \sigma .
\end{aligned}
$$

Proof. It is enough to follow the proof of Proposition 5.7 recalling that $V \equiv 0$ for $N=2$.

The next lemma provides an upper bound for a nonlinear boundary term.

Lemma 5.16. Under the same assumptions of Proposition 5.15, let $\tilde{r}_{1} \in(0, \tilde{R})$ as in Lemma 5.14 with $\varepsilon=1$. Let

$$
g(r):=\frac{\int_{S_{r}}|w|^{p} d \sigma}{\left(\int_{\Omega_{r}}|w(y)|^{p} d y\right)^{\frac{p+2}{2 p}}} .
$$

Then $g \in L^{1}\left(0, \tilde{r}_{1}\right)$ and $g \geqslant 0$ a.e. in $\left(0, \tilde{r}_{1}\right)$. Furthermore

$$
\int_{S_{r}}|w|^{p} d \sigma \leqslant\left(\int_{\Omega_{\tilde{r}_{1}}}|w(y)|^{p} d y\right)^{\frac{p-2}{2 p}} \bar{C}_{1}^{-1} g(r)(D(r)+H(r))
$$

for a.e. $r \in\left(0, \tilde{r}_{1}\right)$. Moreover for any $q>p$ and all $r \in\left(0, \tilde{r}_{1}\right)$, we have

$$
\int_{0}^{r} g(s) d s \leqslant \frac{2 p}{p-2} \pi^{\frac{(q-p)(p-2)}{2 p q}} r^{\frac{(q-p)(p-2)}{p q}}\|w\|_{L^{q}\left(\Omega_{\tilde{r}_{1}}\right)}^{\frac{p-2}{2}} .
$$

Proof. We have

$$
g(r)=\frac{\int_{S_{r}}|w|^{p} d \sigma}{\left(\int_{\Omega_{r}}|w(y)|^{p} d y\right)^{\frac{p+2}{2 p}}}=\frac{d}{d r}\left(\frac{2 p}{p-2}\left(\int_{\Omega_{r}}|w(y)|^{p} d y\right)^{\frac{p-2}{2 p}}\right)
$$


in the distributional sense and for a.e. $r \in\left(0, \tilde{r}_{1}\right)$ and this clearly implies that $g \in L^{1}\left(0, \tilde{r}_{1}\right)$. Furthermore, (130) follows from integration of (131) and Hölder inequality. The proof of (129) follows by Lemma 5.14 and the definition of $g$.

Next we state the two-dimensional version of Lemma 5.9.

Lemma 5.17. The function $D$ defined in (72) belongs to $W_{\mathrm{loc}}^{1,1}(0, \tilde{R})$. Moreover

$$
D(r)+H(r) \geqslant 0 \quad \text { for all } r \in\left(0, \tilde{r}_{1}\right)
$$

where $\tilde{r}_{1} \in(0, \tilde{R})$ is as in Lemma 5.14 with $\varepsilon=1$, and

$$
D^{\prime}(r)=B(r)+\int_{S_{r}} \widetilde{\mathbf{b}} \cdot \nabla w w d \sigma+O\left(r^{-1+\tilde{\delta}}+g(r)\right)(D(r)+H(r))
$$

as $r \rightarrow 0^{+}$, in a distributional sense and for a.e. $r \in\left(0, \tilde{r}_{1}\right)$, where $\tilde{\delta}=\min \{\delta, 4 / p\}$ and

$$
B(r):=2 \int_{S_{r}} \frac{|\widetilde{A} \nabla w \cdot \nu|^{2}}{\mu} d \sigma+\frac{1}{r} \int_{\Gamma_{r}} \frac{|\nabla w|^{2}}{\mu}(\widetilde{A} \tilde{\nu} \cdot \tilde{\nu})(\widetilde{A} y \cdot \tilde{\nu}) d \sigma .
$$

Proof. We give here only a sketch of the proof being essentially similar to the proof Lemma 5.9. By (128), (72), (125), (126), (127), (41), and Lemma 5.14, we obtain

$$
\begin{aligned}
D^{\prime}(r)=B(r) & +\int_{S_{r}}\left(\widetilde{\mathbf{b}} \cdot \nabla w w-\widetilde{h}|w|^{2}-\tilde{f}(y, w) w+2 \widetilde{F}(y, w)\right) d \sigma \\
& +O\left(r^{-1+\tilde{\delta}}\right)(D(r)+H(r)),
\end{aligned}
$$

where $\tilde{\delta}=\min \{\delta, 4 / p\}$. On the other hand, from (41), (42), Lemma $5.14(i),(73),(129)$, we can estimate

$$
\int_{S_{r}}\left(\widetilde{h}|w|^{2}+\tilde{f}(y, w(y)) w-2 \widetilde{F}(y, w)\right) d \sigma=O\left(g(r)+r^{-1+\delta}\right)(D(r)+H(r)) .
$$

In view of (133), (132) yields the conclusion.

We now give the statement of two-dimensional version of Lemma 5.10.

Lemma 5.18. Let $D$ and $H$ be defined in (72-73), $\tilde{r}_{1}$ be as in Lemma 5.14, $\tilde{\delta}$ as in Lemma 5.17, and denote $\Sigma:=\left\{r \in\left(0, \tilde{r}_{1}\right): D^{\prime}(r) H(r) \leqslant H^{\prime}(r) D(r)\right\}$. If $\Sigma \neq \emptyset$ and 0 is a limit point of $\Sigma$, then

$$
D^{\prime}(r)=B(r)+O\left(r^{-1+\tilde{\delta}}+g(r)\right)(D(r)+H(r)) \quad \text { as } r \rightarrow 0^{+}, r \in \Sigma .
$$

Proof. The proof follows that of Lemma 5.10 and exploits Lemma 5.17.

If $\tilde{r}_{1}$ is as in Lemma 5.14 (with $\varepsilon=1$ ), then the function

$$
\mathcal{N}:\left(0, \tilde{r}_{1}\right) \rightarrow \mathbb{R}, \quad \mathcal{N}(r)=\frac{D(r)}{H(r)}
$$

is well defined.

Lemma 5.19. Let $\mathcal{N}:\left(0, \tilde{r}_{1}\right) \rightarrow \mathbb{R}$ be defined in (134). Then the limit $\gamma:=\lim _{r \rightarrow 0^{+}} \mathcal{N}(r)$ exists, is finite and

$$
\gamma \geqslant 0 .
$$

Proof. By Lemma 5.3 and Lemma 5.17 we have that $\mathcal{N} \in W_{\text {loc }}^{1,1}\left(0, \tilde{r}_{1}\right)$ and $\mathcal{N}(r) \geqslant-1$ for all $r \in\left(0, \tilde{r}_{1}\right)$. As explained in the proof of Lemma 5.11 it is not restrictive to assume that $\Sigma \neq \emptyset$ and that 0 is a limit point of $\Sigma$ since otherwise the convergence of $\mathcal{N}$ as $r \rightarrow 0^{+}$is immediate. Therefore by Lemma 5.18 and (85) we obtain

$$
\begin{aligned}
\mathcal{N}^{\prime}(r)= & \frac{2 r\left(\int_{S_{r}} \frac{|\widetilde{A} \nabla w \cdot \nu|^{2}}{\mu} d \sigma\right)\left(\int_{S_{r}} \mu w^{2} d \sigma\right)+\left(\int_{S_{r}} \mu w^{2} d \sigma\right)\left(\int_{\Gamma_{r}} \frac{|\nabla w|^{2}(\tilde{A} \tilde{\nu} \cdot \tilde{\nu})(\widetilde{A} x \cdot \tilde{\nu})}{\mu} d \sigma\right)}{\left(\int_{S_{r}} \mu w^{2} d \sigma\right)^{2}} \\
& -\frac{r}{2} \frac{\left(H^{\prime}(r)\right)^{2}}{H^{2}(r)}+\frac{H^{\prime}(r)}{H(r)} O\left(r^{\delta}\right)+O\left(r^{-1+\tilde{\delta}}+g(r)\right)(\mathcal{N}(r)+1)
\end{aligned}
$$


as $r \rightarrow 0^{+}, r \in \Sigma$. Proceeding as in the proof of Lemma 5.11 we arrive to

$$
\mathcal{N}^{\prime}(r) \geqslant O\left(r^{-1+\tilde{\delta}}+g(r)\right)\left(\mathcal{N}(r)+\frac{1}{2}\right)
$$

By Lemma 5.16 and integration it follows that $\mathcal{N}$ is bounded also from above and, in turn, that $\mathcal{N}^{\prime}$ is the sum of a nonnegative function and of a $L^{1}$-integrable function in a neighborhood of 0 . Therefore $\mathcal{N}$ has a limit as $r \rightarrow 0^{+}$. Finally, (135) follows immediately from Lemma 5.14 (ii).

We conclude this subsection with the following estimates on the function $H$.

Lemma 5.20. There exists $r_{1} \in\left(0, \tilde{r}_{1}\right)$ and $K_{1}>0$ such that

$$
H(r) \leqslant K_{1} r^{2 \gamma} \text { for all } r \in\left(0, r_{1}\right)
$$

and

$$
H(2 r) \leqslant K_{1} H(r) \quad \text { for all } r \in\left(0, r_{1} / 2\right) .
$$

On the other hand for any $\sigma>0$ there exists a constant $K_{2}(\sigma)>0$ depending on $\sigma$ such that

$$
H(r) \geqslant K_{2}(\sigma) r^{2 \gamma+\sigma} \quad \text { for all } r \in\left(0, r_{1}\right) .
$$

Proof. It follows by Lemma 5.19 by proceeding exactly as in the proof of Lemma 5.12.

\section{THE BLOW-UP ARGUMENT}

Throughout this section, we let $\widetilde{A}, \widetilde{\mathbf{b}}, \tilde{f}, \widetilde{h}$ be as in (23-26) with $A, \mathbf{b}, \Psi, f, h, V$ as in assumptions (10-17), (21). Let $\widetilde{\Omega}$ be as in (29) with $\Omega$ satisfying (9) and (2-6). Let $w \in H^{1}(\widetilde{\Omega}) \backslash\{0\}$ be a non-trivial weak solution to $(37)$.

Lemma 6.1. Let $\gamma$ be as in Lemmas 5.11, 5.19 respectively for $N \geqslant 3$ and $N=2$. Then

(i) there exists $k_{0} \in \mathbb{N}$ such that $\gamma=-\frac{N-2}{2}+\sqrt{\left(\frac{N-2}{2}\right)^{2}+\mu_{k_{0}}(V)}$;

(ii) for every sequence $\lambda_{n} \rightarrow 0^{+}$there exists a subsequence $\lambda_{n_{k}}$ and $\psi \in H_{0}^{1}(C) \subset H^{1}\left(\mathbb{S}^{N-1}\right)$ eigenfunction of the operator $\mathcal{L}_{V}=-\Delta_{\mathbb{S}^{N-1}}-V$ associated to the eigenvalue $\mu_{k_{0}}(V)$ such that $\|\psi\|_{L^{2}\left(\mathbb{S}^{N-1}\right)}=1$ and

$$
\frac{w\left(\lambda_{n_{k}} x\right)}{\sqrt{H\left(\lambda_{n_{k}}\right)}} \rightarrow|x|^{\gamma} \psi\left(\frac{x}{|x|}\right)
$$

weakly in $H^{1}\left(B_{1}\right)$, strongly in $C_{\mathrm{loc}}^{1, \alpha}\left(\mathcal{C} \cap B_{1}\right)$ and in $C_{\mathrm{loc}}^{0, \alpha}\left(B_{1} \backslash\{0\}\right)$ for any $\alpha \in(0,1)$, strongly in $H^{1}\left(B_{r}\right)$ for all $r \in(0,1)$, and strongly in $L^{2}\left(\partial B_{1}\right)$, where $w$ is meant to be trivially extended outside $\widetilde{\Omega}$.

Proof. Let us set

$$
w^{\lambda}(x)=\frac{w(\lambda x)}{\sqrt{H(\lambda)}} .
$$

We notice that

$$
\int_{C_{\lambda}} \mu(\lambda \theta)\left(w^{\lambda}(\theta)\right)^{2} d \sigma=1
$$

where $C_{\lambda}$ is defined in (45). If $N \geqslant 3$, by Lemma 5.5 we have that, for all $\lambda \in\left(0, r_{0}\right)$,

$$
\begin{aligned}
\left(\mathcal{N}(\lambda)+\frac{N-2}{2}\right) & \geqslant \frac{\bar{C}}{\lambda^{N-2} H(\lambda)} \int_{\Omega_{\lambda}}\left(|\nabla w(x)|^{2}+\frac{w^{2}(x)}{|x|^{2}}\right) d x \\
& =\bar{C} \int_{\Omega_{\lambda} / \lambda}\left(\left|\nabla w^{\lambda}(x)\right|^{2}+\frac{\left(w^{\lambda}(x)\right)^{2}}{|x|^{2}}\right) d x .
\end{aligned}
$$

Similarly, if $N=2$, by Lemma 5.14 we have that, for all $\lambda \in\left(0, \tilde{r}_{1}\right)$,

$$
\begin{aligned}
(\mathcal{N}(\lambda)+1) & \geqslant \frac{\bar{C}_{1}}{H(\lambda)}\left(\int_{\Omega_{\lambda}}|\nabla w(x)|^{2} d x+\frac{1}{\lambda} \int_{S_{\lambda}} w^{2}\right) \\
& =\bar{C}_{1}\left(\int_{\Omega_{\lambda} / \lambda}\left|\nabla w^{\lambda}(x)\right|^{2} d x+\int_{C_{\lambda}}\left(w^{\lambda}\right)^{2} d \sigma\right) .
\end{aligned}
$$


From (118), Lemma 5.19, (142), and (143), we deduce that the trivial extension

$$
\widetilde{w}^{\lambda}(x)= \begin{cases}w^{\lambda}(x), & \text { if } x \in \Omega_{\lambda} / \lambda \\ 0, & \text { if } x \in B_{1} \backslash \Omega_{\lambda} / \lambda\end{cases}
$$

is bounded in $H^{1}\left(B_{1}\right)$ uniformly with respect to $\lambda \in\left(0, r_{1}\right)$ with $r_{1}$ as in Lemmas 5.12 and 5.20. Therefore, for any given sequence $\lambda_{n} \rightarrow 0^{+}$, there exists a subsequence $\lambda_{n_{k}} \rightarrow 0^{+}$such that $\widetilde{w}^{\lambda_{n_{k}}} \rightarrow \widetilde{w}$ weakly in $H^{1}\left(B_{1}\right)$ and a.e. in $B_{1}$ for some $\widetilde{w} \in H^{1}\left(B_{1}\right)$. Due to compactness of the trace embedding $H^{1}\left(B_{1}\right) \hookrightarrow L^{2}\left(\partial B_{1}\right)$, we obtain that $\widetilde{w}^{\lambda_{n_{k}}} \rightarrow \widetilde{w}$ in $L^{2}\left(\partial B_{1}\right)$ and consequently from (141) $\int_{\partial B_{1}}|\widetilde{w}|^{2} d \sigma=1$. In particular $\widetilde{w} \not \equiv 0$. Moreover $\widetilde{w}=0$ a.e. in $B_{1} \backslash \mathcal{C}$ where $\mathcal{C}$ is defined in (8), as it easily follows from the definition of $\widetilde{w}^{\lambda}$, a.e. convergence of $\widetilde{w}^{\lambda_{n_{k}}} \rightarrow \widetilde{w}$ and the fact

$$
\text { for every } x \in B_{1} \backslash \overline{\mathcal{C}} \text { there exists } \lambda_{x}>0 \text { such that, for all } \lambda \in\left(0, \lambda_{x}\right), x \notin \Omega_{\lambda} / \lambda \text {. }
$$

To prove (144), it is enough to observe that if $x=\left(x^{\prime}, x_{N}\right) \in B_{1} \backslash \overline{\mathcal{C}}$, then $x_{N}<\varphi_{0}\left(x^{\prime}\right)$ and hence from (33) $\lambda x_{N}<\left|x^{\prime}\right| \widetilde{\varphi}\left(\lambda x^{\prime} /\left|x^{\prime}\right|\right)$ for $\lambda$ sufficiently small. From (32), we deduce that $\lambda x /\left|x^{\prime}\right| \notin \widetilde{\Omega}$ for $\lambda$ small which in particular yields $x \notin \Omega_{\lambda} / \lambda$ for $\lambda$ small. This proves claim (144).

By scaling of equation (37), we have that $w^{\lambda}$ weakly solves

$$
\begin{cases}-\operatorname{div}\left(\widetilde{A}(\lambda x) \nabla w^{\lambda}(x)\right)+\lambda \widetilde{\mathbf{b}}(\lambda x) \cdot \nabla w^{\lambda}(x)-\frac{V\left(\frac{x}{|x|}\right)}{|x|^{2}} w^{\lambda}(x) & \\ =\lambda^{2} \widetilde{h}(\lambda x) w^{\lambda}(x)+\frac{\lambda^{2}}{\sqrt{H(\lambda)}} \tilde{f}\left(\lambda x, \sqrt{H(\lambda)} w^{\lambda}(x)\right), & \text { in } \Omega_{\lambda} / \lambda, \\ w^{\lambda}=0, & \text { on } \partial\left(\Omega_{\lambda} / \lambda\right) \cap B_{1} .\end{cases}
$$

In order to pass to the limit in (145), we observe that

$$
\text { if } \xi \in C_{\mathrm{c}}^{\infty}\left(\mathcal{C} \cap B_{1}\right) \text { then } \xi \in C_{\mathrm{c}}^{\infty}\left(\Omega_{\lambda} / \lambda\right) \text { for sufficiently small } \lambda .
$$

Indeed, let us consider $\xi \in C_{\mathrm{c}}^{\infty}\left(\mathcal{C} \cap B_{1}\right)$ and denote $K=\operatorname{supp} \xi$. Since $K$ is compact, we have that

$$
\tau=\min _{\left(x^{\prime}, x_{N}\right) \in K}\left(x_{N}-\varphi_{0}\left(x^{\prime}\right)\right)>0 .
$$

From (33), there exists $t_{0}$ such that

$$
\left|\frac{\widetilde{\varphi}(t \nu)}{t}-g(\nu)\right|<\tau \text { for all } t \in\left(0, t_{0}\right) \text { and for all } \nu \in \mathbb{S}^{N-2} .
$$

Then for all $\lambda \in\left(0, t_{0}\right)$ and $\left(x^{\prime}, x_{N}\right) \in K$ we have that

$$
\lambda x_{N}-\widetilde{\varphi}\left(\lambda x^{\prime}\right)=\lambda\left(x_{N}-\varphi_{0}\left(x^{\prime}\right)\right)+\lambda\left|x^{\prime}\right|\left(g\left(x^{\prime} /\left|x^{\prime}\right|\right)-\frac{\widetilde{\varphi}\left(\lambda\left|x^{\prime}\right| \frac{x^{\prime}}{\left|x^{\prime}\right|}\right)}{\lambda\left|x^{\prime}\right|}\right)>0
$$

and hence, by (32), $K \subset \Omega_{\lambda} / \lambda$ for all $\lambda \in\left(0, t_{0}\right)$, thus proving claim (146). Hence we can test equation (145) with every $\xi \in C_{\mathrm{c}}^{\infty}\left(\mathcal{C} \cap B_{1}\right)$. From (38) we have that

$$
\int_{\Omega_{\lambda_{n_{k}} / \lambda_{n_{k}}}} \widetilde{A}\left(\lambda_{n_{k}} x\right) \nabla w^{\lambda_{n_{k}}}(x) \cdot \nabla \xi(x) d x=\int_{\mathcal{C} \cap B_{1}} \nabla \widetilde{w}(x) \cdot \nabla \xi(x) d x+o(1) \quad \text { as } k \rightarrow+\infty .
$$

From (39) and (42)

$$
\lambda_{n_{k}} \int_{\Omega_{\lambda_{n_{k}} / \lambda_{n_{k}}}} \widetilde{\mathbf{b}}\left(\lambda_{n_{k}} x\right) \cdot \nabla w^{\lambda_{n_{k}}}(x) \xi(x) d x-\lambda_{n_{k}}^{2} \int_{\Omega_{\lambda_{n_{k}} / \lambda_{n_{k}}}} \widetilde{h}\left(\lambda_{n_{k}} x\right) w^{\lambda_{n_{k}}}(x) \xi(x) d x=o(1)
$$

as $k \rightarrow+\infty$. From (41) and Hölder and Sobolev inequalities, we have that, denoting $\tilde{p}=2^{*}$ if $N \geqslant 3$ and $\tilde{p}=p$ with $p$ as in (17) if $N=2$,

$$
\begin{aligned}
& \frac{\lambda_{n_{k}}^{2}}{\sqrt{H\left(\lambda_{n_{k}}\right)}}\left|\int_{\Omega_{\lambda_{n_{k}} / \lambda_{n_{k}}}} \tilde{f}\left(\lambda_{n_{k}} x, \sqrt{H\left(\lambda_{n_{k}}\right)} w^{\lambda_{n_{k}}}(x)\right) \xi(x) d x\right| \\
& \leqslant C_{\tilde{f}} \lambda_{n_{k}}^{2} \int_{\Omega_{\lambda_{n_{k}} / \lambda_{n_{k}}}}\left|w^{\lambda_{n_{k}}}(x)\right||\xi(x)| d x+C_{\tilde{f}} \lambda_{n_{k}}^{2} \int_{\Omega_{\lambda_{n_{k}} / \lambda_{n_{k}}}}\left|w\left(\lambda_{n_{k}} x\right)\right|^{\tilde{p}-2}\left|w^{\lambda_{n_{k}}}(x)\right||\xi(x)| d x \\
& \leqslant C_{\tilde{f}} \lambda_{n_{k}}^{2}\left\|\widetilde{w}^{\lambda_{n_{k}}}\right\|_{H^{1}\left(B_{1}\right)}\|\xi\|_{H^{1}\left(B_{1}\right)}+C_{\tilde{f}} \lambda_{n_{k}}^{2-N+\frac{2 N}{\tilde{p}}}\left\|\widetilde{w}^{\lambda_{n_{k}}}\right\|_{L^{\tilde{p}}\left(B_{1}\right)}\|\xi\|_{L^{\tilde{p}}\left(B_{1}\right)}\|w\|_{L^{\tilde{p}}\left(\Omega_{\lambda_{n_{k}}}\right)}^{\tilde{p}-2}=o(1)
\end{aligned}
$$


as $k \rightarrow+\infty$. Testing equation (145) with $\xi \in C_{\mathrm{c}}^{\infty}\left(\mathcal{C} \cap B_{1}\right)$, letting $k \rightarrow+\infty$, and using (147-149), we obtain that $\widetilde{w}$ is a weak solution to

$$
\begin{cases}-\Delta \widetilde{w}-\frac{V\left(\frac{x}{|x|}\right)}{|x|^{2}} \widetilde{w}=0, & \text { in } \mathcal{C} \cap B_{1}, \\ \widetilde{w}=0, & \text { on } \partial \mathcal{C} \cap B_{1} .\end{cases}
$$

For $\lambda \in\left(0, r_{1}\right)$ we define

$$
\Psi_{\lambda}:\left\{\left(y^{\prime}, y_{N}\right) \in \mathbb{R}^{N-1} \times \mathbb{R}:\left|y^{\prime}\right| \leqslant 1\right\} \rightarrow\left\{\left(y^{\prime}, y_{N}\right) \in \mathbb{R}^{N-1} \times \mathbb{R}:\left|y^{\prime}\right| \leqslant 1\right\}
$$

as

$$
\Psi_{\lambda}\left(y^{\prime}, y_{N}\right):=\left(y^{\prime}, y_{N}+\frac{\widetilde{\varphi}\left(\lambda y^{\prime}\right)}{\lambda}\right) .
$$

We notice that $\Psi_{\lambda}$ is invertible and $\Psi_{\lambda}^{-1}\left(x^{\prime}, x_{N}\right):=\left(x^{\prime}, x_{N}-\frac{\widetilde{\varphi}\left(\lambda x^{\prime}\right)}{\lambda}\right)$. Let us fix $r \in(0,1)$, $s_{1}, s_{2}, \rho_{1}, \rho_{2}, R_{1}, R_{2}$ such that $0<R_{1}<\rho_{1}<s_{1}<r<s_{2}<\rho_{2}<R_{2}<1$, and denote

$$
\begin{aligned}
& \mathcal{A}_{R_{1}, R_{2}}:=\left\{\left(y^{\prime}, y_{N}\right) \in \mathbb{R}^{N-1} \times(0,+\infty): R_{1}<\sqrt{\left|y^{\prime}\right|^{2}+\left(y_{N}+\varphi_{0}\left(y^{\prime}\right)\right)^{2}}<R_{2}\right\}, \\
& \mathcal{A}_{\rho_{1}, \rho_{2}}:=\left\{\left(y^{\prime}, y_{N}\right) \in \mathbb{R}^{N-1} \times(0,+\infty): \rho_{1}<\sqrt{\left|y^{\prime}\right|^{2}+\left(y_{N}+\varphi_{0}\left(y^{\prime}\right)\right)^{2}}<\rho_{2}\right\} .
\end{aligned}
$$

Using (33) it is easy to verify that there exist $\lambda_{0} \in\left(0, r_{1}\right)$ and $c_{0}>0$ such that for all $\lambda \in\left(0, \lambda_{0}\right)$

$$
\left|\Psi_{\lambda}(y)\right| \geqslant c_{0} \quad \text { for every } y \in \mathcal{A}_{R_{1}, R_{2}}
$$

and

$$
\begin{aligned}
& \left\{\left(y^{\prime}, y_{N}\right) \in \mathbb{R}^{N-1} \times(0,+\infty): \sqrt{\left|y^{\prime}\right|^{2}+\left(y_{N}+\lambda^{-1} \widetilde{\varphi}\left(\lambda y^{\prime}\right)\right)^{2}}=r\right\} \\
& \subseteq\left\{\left(y^{\prime}, y_{N}\right) \in \mathbb{R}^{N-1} \times(0,+\infty): s_{1}<\sqrt{\left|y^{\prime}\right|^{2}+\left(y_{N}+\lambda^{-1} \widetilde{\varphi}\left(\lambda y^{\prime}\right)\right)^{2}}<s_{2}\right\} \subseteq \mathcal{A}_{\rho_{1}, \rho_{2}} \\
& \subseteq \mathcal{A}_{R_{1}, R_{2}} \subseteq\left\{\left(y^{\prime}, y_{N}\right) \in \mathbb{R}^{N-1} \times(0,+\infty): \sqrt{\left|y^{\prime}\right|^{2}+\left(y_{N}+\lambda^{-1} \widetilde{\varphi}\left(\lambda y^{\prime}\right)\right)^{2}}<1\right\}
\end{aligned}
$$

namely

$$
\Psi_{\lambda}^{-1}\left(\frac{\Omega_{\lambda}}{\lambda} \cap \partial B_{r}\right) \subseteq \Psi_{\lambda}^{-1}\left(\frac{\Omega_{\lambda}}{\lambda} \cap\left(B_{s_{2}} \backslash \bar{B}_{s_{1}}\right)\right) \subseteq \mathcal{A}_{\rho_{1}, \rho_{2}} \subseteq \mathcal{A}_{R_{1}, R_{2}} \subseteq \Psi_{\lambda}^{-1}\left(\frac{\Omega_{\lambda}}{\lambda}\right)
$$

for all $\lambda \in\left(0, \lambda_{0}\right)$. From (145) and (152), the functions $v^{\lambda}(y):=w^{\lambda}\left(\Psi_{\lambda}(y)\right)$ satisfy

$$
\left\{\begin{array}{cl}
-\operatorname{div}\left(\widetilde{A}^{\lambda}(y) \nabla v^{\lambda}(y)\right)+\widetilde{\mathbf{b}}^{\lambda}(y) \cdot \nabla v^{\lambda}(y)-\frac{V\left(\frac{\Psi_{\lambda}(y)}{|\Psi \lambda(y)|}\right)}{\left|\Psi_{\lambda}(y)\right|^{2}} v^{\lambda}(y) \\
=\widetilde{h}^{\lambda}(y) v^{\lambda}(y)+\tilde{f}^{\lambda}\left(y, v^{\lambda}(y)\right), & \text { in } \mathcal{A}_{R_{1}, R_{2}}, \\
v^{\lambda}=0, & \text { on } \partial \mathcal{A}_{R_{1}, R_{2}} \cap\left\{\left(y^{\prime}, y_{N}\right): y_{N}=0\right\},
\end{array}\right.
$$

for all $\lambda \in\left(0, \lambda_{0}\right)$, where

$$
\begin{aligned}
& \widetilde{A}^{\lambda}(y)=\left(\operatorname{Jac} \Psi_{\lambda}(y)\right)^{-1} \widetilde{A}\left(\lambda \Psi_{\lambda}(y)\right)\left(\left(\operatorname{Jac} \Psi_{\lambda}(y)\right)^{T}\right)^{-1}, \quad \widetilde{\mathbf{b}}^{\lambda}(y)=\lambda \widetilde{\mathbf{b}}\left(\lambda \Psi_{\lambda}(y)\right)\left(\left(\operatorname{Jac} \Psi_{\lambda}(y)\right)^{T}\right)^{-1}, \\
& \tilde{f}^{\lambda}(y, s)=\frac{\lambda^{2}}{\sqrt{H(\lambda)}} \tilde{f}\left(\lambda \Psi_{\lambda}(y), \sqrt{H(\lambda)} s\right), \quad \widetilde{h}^{\lambda}(y)=\lambda^{2} \widetilde{h}\left(\lambda \Psi_{\lambda}(y)\right) .
\end{aligned}
$$

From (36) $\left\|\widetilde{A}^{\lambda}\right\|_{W^{1, \infty}\left(\mathcal{A}_{R_{1}, R_{2}}\right)}$ is bounded uniformly with respect to $\lambda \in\left(0, \lambda_{0}\right)$. From (39) and (42) $\left\|\widetilde{\mathbf{b}}^{\lambda}\right\|_{L^{\infty}\left(\mathcal{A}_{R_{1}, R_{2}}\right)},\left\|\widetilde{h}^{\lambda}\right\|_{L^{\infty}\left(\mathcal{A}_{R_{1}, R_{2}}\right)}$ are bounded uniformly with respect to $\lambda \in\left(0, \lambda_{0}\right)$. From (41), Lemmas 5.12 and 5.20, (111), and (135), we have that, denoting again $\tilde{p}=2^{*}$ if $N \geqslant 3$ and $\tilde{p}=p$ with $p$ as in (17) if $N=2$,

$$
\begin{aligned}
\left|\frac{\tilde{f}^{\lambda}\left(y, v^{\lambda}(y)\right)}{v^{\lambda}(y)}\right| & \leqslant C_{\tilde{f}} \lambda^{2}\left(1+|H(\lambda)|^{(\tilde{p}-2) / 2}\left|v^{\lambda}(y)\right|^{\tilde{p}-2}\right) \\
& \leqslant \operatorname{const}\left(\lambda^{2}+\lambda^{(\tilde{p}-2)\left(\frac{2}{\tilde{p}-2}+\gamma\right)}\left|v^{\lambda}(y)\right|^{\tilde{p}-2}\right) \leqslant \operatorname{const}\left(1+\left|v^{\lambda}(y)\right|^{\tilde{p}-2}\right) .
\end{aligned}
$$


Hence, if we define $s=q /(\tilde{p}-2)>N / 2$ with $q$ as in Proposition 4.1 if $N \geqslant 3$ and $q>\tilde{p}-2$ if $N=2$, then by (35) and two changes of variables, we obtain

$$
\begin{aligned}
\left\|\frac{\tilde{f}^{\lambda}\left(y, v^{\lambda}(y)\right)}{v^{\lambda}(y)}\right\|_{L^{s}\left(\mathcal{A}_{R_{1}, R_{2}}\right)} & \leqslant \operatorname{const}\left[1+\lambda^{2}|H(\lambda)|^{\frac{\tilde{p}-2}{2}}\left(\int_{\mathcal{A}_{R_{1}, R_{2}}}\left|v^{\lambda}(y)\right|^{(\tilde{p}-2) s} d y\right)^{1 / s}\right] \\
& \leqslant \operatorname{const}\left[1+\lambda^{2}|H(\lambda)|^{\frac{\tilde{p}-2}{2}}\left(\int_{B_{1}}\left|\widetilde{w}^{\lambda}(x)\right|^{q}\left|\operatorname{det} \operatorname{Jac}_{\lambda}^{-1}(x)\right| d x\right)^{1 / s}\right] \\
& \leqslant \text { const }\left[1+\lambda^{2-\frac{N}{s}}\left(\int_{\Omega_{\lambda}}|w(x)|^{q} d x\right)^{1 / s}\right]=O(1) \quad \text { as } \lambda \rightarrow 0^{+} .
\end{aligned}
$$

Furthermore, up to shrinking $\lambda_{0}$, it is easy to verify that $\left\{v^{\lambda}\right\}_{\lambda \in\left(0, \lambda_{0}\right)}$ is bounded in $H^{1}\left(\mathcal{A}_{R_{1}, R_{2}}\right)$ uniformly with respect to $\lambda$ and that

$$
\inf _{\substack{\lambda \in\left(0, \lambda_{0}\right) \\ y \in \mathcal{A}_{R_{1}, R_{2}}}} \inf _{\xi \in \mathbb{R}^{N} \backslash\{0\}} \frac{\widetilde{A}^{\lambda}(y) \xi \cdot \xi}{|\xi|^{2}}>0 .
$$

Therefore, using classical iterative estimates of Brezis-Kato [4] type (see also Proposition 4.1), standard bootstrap, elliptic regularity theory, (152), (153), we first deduce that

$$
\left\{v^{\lambda}\right\}_{\lambda \in\left(0, \lambda_{0}\right)} \text { is bounded in } C^{1, \alpha}\left(\mathcal{A}_{\rho_{1}, \rho_{2}}\right) \text { uniformly with respect to } \lambda \text { for all } \alpha \in(0,1) \text {. }
$$

From (154) and local Lipschitz continuity of $\widetilde{\varphi}$, it follows that, for all $x, z \in \Psi_{\lambda}\left(\mathcal{A}_{\rho_{1}, \rho_{2}}\right)$,

$$
\begin{aligned}
\left|w^{\lambda}(x)-w^{\lambda}(z)\right| & =\left|v^{\lambda}\left(\Psi_{\lambda}^{-1}(x)\right)-v^{\lambda}\left(\Psi_{\lambda}^{-1}(z)\right)\right| \leqslant\left\|v^{\lambda}\right\|_{C^{0, \alpha}\left(\mathcal{A}_{\rho_{1}, \rho_{2}}\right)}\left|\Psi_{\lambda}^{-1}(x)-\Psi_{\lambda}^{-1}(z)\right|^{\alpha} \\
& \leqslant\left\|v^{\lambda}\right\|_{C^{0, \alpha}\left(\mathcal{A}_{\rho_{1}, \rho_{2}}\right)}\left(|x-z|+\frac{\left|\widetilde{\varphi}\left(\lambda x^{\prime}\right)-\widetilde{\varphi}\left(\lambda z^{\prime}\right)\right|}{\lambda}\right)^{\alpha} \\
& \leqslant \text { const }\left\|v^{\lambda}\right\|_{C^{0, \alpha}\left(\mathcal{A}_{\rho_{1}, \rho_{2}}\right)}|x-z|^{\alpha},
\end{aligned}
$$

while from (154) and (36) we deduce

$$
\begin{aligned}
& \left|\nabla w^{\lambda}(x)-\nabla w^{\lambda}(z)\right| \leqslant\left|\left(\nabla v^{\lambda}\left(\Psi_{\lambda}^{-1}(x)\right)-\nabla v^{\lambda}\left(\Psi_{\lambda}^{-1}(z)\right)\right) \operatorname{Jac} \Psi_{\lambda}^{-1}(x)\right| \\
& +\left|\nabla v^{\lambda}\left(\Psi_{\lambda}^{-1}(z)\right)\left(\operatorname{Jac} \Psi_{\lambda}^{-1}(x)-\operatorname{Jac} \Psi_{\lambda}^{-1}(z)\right)\right| \\
& \leqslant \operatorname{const}\left(\left\|\nabla v^{\lambda}\right\|_{C^{0, \alpha}\left(\mathcal{A}_{\rho_{1}, \rho_{2}}\right)}\left|\Psi_{\lambda}^{-1}(x)-\Psi_{\lambda}^{-1}(z)\right|^{\alpha}+|| \nabla v^{\lambda} \|_{L^{\infty}\left(\mathcal{A}_{\rho_{1}, \rho_{2}}\right)}\left|\nabla \widetilde{\varphi}\left(\lambda x^{\prime}\right)-\nabla \widetilde{\varphi}\left(\lambda z^{\prime}\right)\right|\right) \\
& \leqslant \operatorname{const}|x-z|^{\alpha} .
\end{aligned}
$$

In particular, the above estimates yield that $\left\|w^{\lambda}\right\|_{C^{1, \alpha}\left(\Psi_{\lambda}\left(\mathcal{A}_{\rho_{1}, \rho_{2}}\right)\right)}$ is bounded uniformly with respect to $\lambda \in\left(0, \lambda_{0}\right)$ for all $\alpha \in(0,1)$, and hence, taking into account (152),

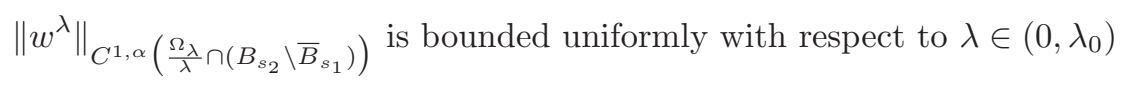

for all $\alpha \in(0,1)$. (155) implies that $\left\|\widetilde{w}^{\lambda}\right\|_{C^{0, \alpha}\left(B_{s_{2}} \backslash \bar{B}_{s_{1}}\right)}$ is bounded uniformly with respect to $\lambda \in\left(0, \lambda_{0}\right)$, and hence

$$
\left\{\widetilde{w}^{\lambda}\right\}_{\lambda \in\left(0, \lambda_{0}\right)} \text { is relatively compact in } C^{0, \alpha}\left(B_{s_{2}} \backslash \bar{B}_{s_{1}}\right),
$$

for all $\alpha \in(0,1)$. From (151) and (155) it follows that

$$
\left\{\nabla \widetilde{w}^{\lambda}\right\}_{\lambda \in\left(0, \lambda_{0}\right)} \text { is relatively compact in } L^{2}\left(\partial B_{r}\right) .
$$

and consequently, from weak convergence $\widetilde{w}^{\lambda_{n_{k}}} \rightarrow \widetilde{w}$ in $H^{1}\left(B_{1}\right)$ we deduce that for every $r \in(0,1)$

$$
\nabla \widetilde{w}^{\lambda_{n_{k}}} \rightarrow \nabla \widetilde{w} \text { in } L^{2}\left(\partial B_{r}\right) .
$$

From (156), (155), and compact embedding of Hölder spaces, reasoning as in the proof of (146), we also obtain that for all $\alpha \in(0,1)$

$$
\widetilde{w}^{\lambda_{n_{k}}} \rightarrow \widetilde{w} \text { in } C_{\text {loc }}^{0, \alpha}\left(B_{1} \backslash\{0\}\right) \quad \text { and } \quad w^{\lambda_{n_{k}}} \rightarrow \widetilde{w} \text { in } C_{\text {loc }}^{1, \alpha}\left(\mathcal{C} \cap B_{1}\right) .
$$


Testing equation (145) with $w^{\lambda}$ and integrating over $\left(\Omega_{\lambda} / \lambda\right) \cap B_{r}$ with $r \in(0,1)$, we obtain

$$
\begin{gathered}
\text { (160) } \int_{\left(\Omega_{\lambda} / \lambda\right) \cap B_{r}} \widetilde{A}(\lambda x) \nabla w^{\lambda}(x) \cdot \nabla w^{\lambda}(x) d x+\lambda \int_{\left(\Omega_{\lambda} / \lambda\right) \cap B_{r}} \widetilde{\mathbf{b}}(\lambda x) \cdot \nabla w^{\lambda}(x) w^{\lambda}(x) d x \\
-\int_{\left(\Omega_{\lambda} / \lambda\right) \cap B_{r}} \frac{V\left(\frac{x}{|x|}\right)}{|x|^{2}}\left|w^{\lambda}(x)\right|^{2} d x=\lambda^{2} \int_{\left(\Omega_{\lambda} / \lambda\right) \cap B_{r}} \tilde{h}(\lambda x)\left|w^{\lambda}(x)\right|^{2} d x \\
+\frac{\lambda^{2}}{\sqrt{H(\lambda)}} \int_{\left(\Omega_{\lambda} / \lambda\right) \cap B_{r}} \tilde{f}\left(\lambda x, \sqrt{H(\lambda)} w^{\lambda}(x)\right) w^{\lambda}(x) d x+\int_{\left(\Omega_{\lambda} / \lambda\right) \cap \partial B_{r}} \widetilde{A}(\lambda x) \nabla w^{\lambda}(x) \cdot \nu(x) w^{\lambda}(x) d \sigma(x) .
\end{gathered}
$$

From (38) and boundedness of $\left\{\widetilde{w}^{\lambda}\right\}_{\lambda \in\left(0, \lambda_{0}\right)}$ in $H^{1}\left(B_{1}\right)$ we have that

$$
\int_{\left(\Omega_{\lambda} / \lambda\right) \cap B_{r}} \tilde{A}(\lambda x) \nabla w^{\lambda}(x) \cdot \nabla w^{\lambda}(x) d x=\int_{\left(\Omega_{\lambda} / \lambda\right) \cap B_{r}}\left|\nabla w^{\lambda}(x)\right|^{2} d x+O\left(\lambda^{\delta}\right) \quad \text { as } \lambda \rightarrow 0^{+} .
$$

From (39) and (42) we have that

$$
\lambda \int_{\left(\Omega_{\lambda} / \lambda\right) \cap B_{r}} \widetilde{\mathbf{b}}(\lambda x) \cdot \nabla w^{\lambda}(x) w^{\lambda}(x) d x=O\left(\lambda^{\delta}\right) \quad \text { as } \lambda \rightarrow 0^{+}
$$

and

$$
\lambda^{2} \int_{\left.\left(\Omega_{\lambda} / \lambda\right)\right) \cap B_{r}} \widetilde{h}(\lambda x)\left|w^{\lambda}(x)\right|^{2} d x=O\left(\lambda^{\delta}\right) \quad \text { as } \lambda \rightarrow 0^{+} .
$$

Proceeding as in (149) we can prove that

$$
\frac{\lambda^{2}}{\sqrt{H(\lambda)}} \int_{\left(\Omega_{\lambda} / \lambda\right) \cap B_{r}} \tilde{f}\left(\lambda x, \sqrt{H(\lambda)} w^{\lambda}(x)\right) w^{\lambda}(x) d x=o(1) \quad \text { as } \lambda \rightarrow 0^{+} .
$$

By (160-164), (38), and (155), we obtain

$$
\int_{\left(\Omega_{\lambda} / \lambda\right) \cap B_{r}}\left(\left|\nabla w^{\lambda}(x)\right|^{2}-\frac{V\left(\frac{x}{|x|}\right)}{|x|^{2}}\left|w^{\lambda}(x)\right|^{2}\right) d x=\int_{\left(\Omega_{\lambda} / \lambda\right) \cap \partial B_{r}} \frac{\partial w^{\lambda}}{\partial \nu} w^{\lambda} d \sigma+o(1)
$$

as $\lambda \rightarrow 0^{+}$, so that, along the sequence $\lambda_{n_{k}}$, by (158), the strong convergence $\widetilde{w}^{\lambda_{n_{k}}} \rightarrow \widetilde{w}$ in $L^{2}\left(\partial B_{r}\right)$, and (150), we obtain for any positive constant $\widetilde{C}$

$$
\begin{aligned}
& \lim _{k \rightarrow+\infty}\left(\int _ { ( \Omega _ { \lambda _ { n _ { k } } / \lambda _ { n _ { k } } } ) \cap B _ { r } } \left(\left|\nabla w^{\lambda_{n_{k}}}(x)\right|^{2} d x-\frac{V\left(\frac{x}{|x|}\right)}{|x|^{2}} \mid w^{\left.\left.\lambda_{n_{k}}(x)\right|^{2}\right) d x}\right.\right. \\
& \left.\quad+\frac{\widetilde{C}}{r} \int_{\left(\Omega_{\lambda_{n_{k}}} / \lambda_{n_{k}}\right) \cap \partial B_{r}}\left|w^{\lambda_{n_{k}}}(x)\right|^{2} d \sigma(x)\right) \\
& =\int_{\mathcal{C} \cap \partial B_{r}} \frac{\partial \widetilde{w}}{\partial \nu}(x) \widetilde{w}(x) d \sigma(x)+\frac{\widetilde{C}}{r} \int_{\mathcal{C} \cap \partial B_{r}}|\widetilde{w}(x)|^{2} d \sigma(x) \\
& =\int_{\mathcal{C} \cap B_{r}}|\nabla \widetilde{w}(x)|^{2} d x-\int_{\mathcal{C} \cap B_{r}} \frac{V\left(\frac{x}{|x|}\right)}{|x|^{2}}|\widetilde{w}(x)|^{2} d x+\frac{\widetilde{C}}{r} \int_{\mathcal{C} \cap \partial B_{r}}|\widetilde{w}(x)|^{2} d \sigma(x)
\end{aligned}
$$

as $k \rightarrow+\infty$ and consequently

$$
\begin{aligned}
\lim _{k \rightarrow+\infty}\left(\int_{\left(\Omega_{\lambda_{n_{k}} / \lambda_{n_{k}}} \cup \mathcal{C}\right) \cap B_{r}}\left(\left|\nabla\left(\widetilde{w}^{\lambda_{n_{k}}}-\widetilde{w}\right)(x)\right|^{2} d x-\frac{V\left(\frac{x}{|x|}\right)}{|x|^{2}}\left(\widetilde{w}^{\lambda_{n_{k}}}-\widetilde{w}\right)^{2}(x)\right) d x\right. \\
+\frac{\widetilde{C}}{r} \int_{\left(\Omega_{\lambda_{n_{k}} / \lambda_{n_{k}}} \cup \mathcal{C}\right) \cap \partial B_{r}}\left(\widetilde{w}^{\left.\left.\lambda_{n_{k}}-\widetilde{w}\right)^{2}(x) d \sigma(x)\right)=0}\right.
\end{aligned}
$$

which, in view of Corollary 3.5 with $\lambda r$ in place of $r$ and through the change of variable $y=\lambda x$ yields the strong convergence

$$
\widetilde{w}^{\lambda_{n_{k}}} \rightarrow \widetilde{w} \quad \text { in } H^{1}\left(B_{r}\right) .
$$


According to (145) we define the functions

$$
\begin{aligned}
& D_{k}(r) \\
& =\frac{1}{r^{N-2}} \int_{\left(\Omega_{\lambda_{n_{k}}} / \lambda_{n_{k}}\right) \cap B_{r}}\left(\widetilde{A}\left(\lambda_{n_{k}} y\right) \nabla w^{\lambda_{n_{k}}}(y) \cdot \nabla w^{\lambda_{n_{k}}}(y)+\lambda_{n_{k}} \widetilde{\mathbf{b}}\left(\lambda_{n_{k}} y\right) \cdot \nabla w^{\lambda_{n_{k}}}(y) w^{\lambda_{n_{k}}}(y)\right) d y \\
& \quad-\frac{1}{r^{N-2}} \int_{\left(\Omega_{\lambda_{n_{k}} / \lambda_{n_{k}}}\right) \cap B_{r}}\left(\frac{V\left(\frac{y}{|y|}\right)}{|y|^{2}}\left|w^{\lambda_{n_{k}}}(y)\right|^{2}+\lambda_{n_{k}}^{2} \widetilde{h}\left(\lambda_{n_{k}} y\right)\left|w^{\lambda_{n_{k}}}(y)\right|^{2}\right) d y \\
& \quad-\frac{\lambda_{n_{k}}^{2}}{r^{N-2} \sqrt{H\left(\lambda_{n_{k}}\right)}} \int_{\left(\Omega_{\lambda_{n_{k}}} / \lambda_{n_{k}}\right) \cap B_{r}} \tilde{f}\left(\lambda_{n_{k}} y, \sqrt{H\left(\lambda_{n_{k}}\right)} w^{\lambda_{n_{k}}}(y)\right) w^{\lambda_{n_{k}}}(y) d y \\
& H_{k}(r)=\frac{1}{r^{N-1}} \int_{\left(\Omega_{\lambda_{n_{k}}} / \lambda_{n_{k}}\right) \cap \partial B_{r}} \mu\left(\lambda_{n_{k}} y\right)\left|w^{\lambda_{n_{k}}}(y)\right|^{2} d \sigma(y) .
\end{aligned}
$$

By (161-165) we infer that, for any $r \in(0,1)$,

$$
D_{k}(r) \rightarrow D_{\widetilde{w}}(r) \quad \text { and } \quad H_{k}(r) \rightarrow H_{\widetilde{w}}(r)
$$

as $k \rightarrow+\infty$ where

$$
D_{\widetilde{w}}(r):=\frac{1}{r^{N-2}} \int_{\mathcal{C} \cap B_{r}}\left(|\nabla \widetilde{w}(y)|^{2}-\frac{V\left(\frac{y}{|y|}\right)}{|y|^{2}} \widetilde{w}^{2}(y)\right) d y \quad \text { and } \quad H_{\widetilde{w}}(r):=\frac{1}{r^{N-1}} \int_{\mathcal{C} \cap \partial B_{r}} \widetilde{w}^{2} d \sigma .
$$

By (150) and (14) we have that $H_{\widetilde{w}}(r)>0$ for all $r \in(0,1)$. Therefore the function

$$
\mathcal{N}_{\widetilde{w}}(r):=\frac{D_{\widetilde{w}}(r)}{H_{\widetilde{w}}(r)} \quad \text { for any } r \in(0,1)
$$

is well defined. Moreover by direct computation one verifies that

$$
\frac{D_{k}(r)}{H_{k}(r)}=\mathcal{N}\left(\lambda_{n_{k}} r\right)
$$

for all $r \in(0,1)$. By $(166-168)$, Lemmas 5.11 and 5.19 , letting $k \rightarrow+\infty$, we obtain

$$
\mathcal{N}_{\widetilde{w}}(r)=\gamma \quad \text { for all } r \in(0,1),
$$

where $\gamma$ is as in Lemmas 5.11 and 5.19.

Proceeding as in Propositions 5.7 and 5.15, Lemmas 5.9, 5.17 and 5.4, and taking into account that $\widetilde{w}$ solves problem $(150)$ in the domain $\mathcal{C} \cap B_{1}$, we deduce that $D_{\widetilde{w}}, H_{\widetilde{w}}, \mathcal{N}_{\widetilde{w}} \in W_{\text {loc }}^{1,1}(0,1)$ and

$$
\begin{aligned}
D_{\widetilde{w}}^{\prime}(r) & =2 r^{2-N} \int_{\mathcal{C} \cap \partial B_{r}}\left|\frac{\partial \widetilde{w}}{\partial \nu}\right|^{2} d \sigma=2 r^{2-N} \int_{\partial B_{r}}\left|\frac{\partial \widetilde{w}}{\partial \nu}\right|^{2} d \sigma \\
H_{\widetilde{w}}^{\prime}(r) & =2 r^{1-N} \int_{\mathcal{C} \cap \partial B_{r}} \frac{\partial \widetilde{w}}{\partial \nu} \widetilde{w} d \sigma=2 r^{1-N} \int_{\partial B_{r}} \frac{\partial \widetilde{w}}{\partial \nu} \widetilde{w} d \sigma \\
D_{\widetilde{w}}(r) & =\frac{r}{2} H_{\widetilde{w}}^{\prime}(r), \\
\mathcal{N}_{\widetilde{w}}^{\prime}(r) & =\frac{2 r\left[\left(\int_{\partial B_{r}}\left|\frac{\partial \widetilde{w}}{\partial \nu}\right|^{2} d \sigma\right)\left(\int_{\partial B_{r}} \widetilde{w}^{2} d \sigma\right)-\left(\int_{\partial B_{r}} \frac{\partial \widetilde{w}}{\partial \nu} \widetilde{w} d \sigma\right)^{2}\right]}{\left(\int_{\partial B_{r}} \widetilde{w}^{2} d \sigma\right)^{2}}
\end{aligned}
$$

for a.e. $r \in(0,1)$. On the other hand, by $(169), \mathcal{N}_{\widetilde{w}}$ is a constant function thus implying

$$
\left(\int_{\partial B_{r}}\left|\frac{\partial \widetilde{w}}{\partial \nu}\right|^{2} d \sigma\right)\left(\int_{\partial B_{r}} \widetilde{w}^{2} d \sigma\right)-\left(\int_{\partial B_{r}} \frac{\partial \widetilde{w}}{\partial \nu} \widetilde{w} d \sigma\right)^{2}=0
$$

The above identity shows that the functions $\frac{\partial \widetilde{w}}{\partial \nu}$ and $\widetilde{w}$ have the same direction as vectors in $L^{2}\left(\partial B_{r}\right)$ and hence there exists a function $\eta=\eta(r)$ such that

$$
\frac{\partial \widetilde{w}}{\partial \nu}(r, \theta)=\eta(r) \widetilde{w}(r, \theta) \quad \text { for a.e. } r \in(0,1), \theta \in \mathbb{S}^{N-1} \text {. }
$$

Since necessarily $\eta(r)=\frac{H_{\widetilde{w}}^{\prime}(r)}{2 H_{\widetilde{w}}(r)}$, then $\eta \in L_{\text {loc }}^{1}(0,1)$. Integration of (170) yields

$$
\widetilde{w}(r, \theta)=e^{\int_{1}^{r} \eta(s) d s} \widetilde{w}(1, \theta)=\varphi(r) \psi(\theta) \quad \text { for all } r \in(0,1), \theta \in \mathbb{S}^{N-1},
$$


where $\varphi(r)=e^{\int_{1}^{r} \eta(s) d s}$ and $\psi(\theta)=\widetilde{w}(1, \theta)$. We notice that $\psi \in H_{0}^{1}(C)$ and (150) may be written in polar coordinates as

$$
\left(-\varphi^{\prime \prime}(r)-\frac{N-1}{r} \varphi^{\prime}(r)\right) \psi(\theta)-\frac{\varphi(r)}{r^{2}} \mathcal{L}_{V} \psi(\theta)=0 \quad \text { in }(0,1) \times C .
$$

Taking $r$ fixed we may observe that $\psi$ has to be necessarily an eigenfunction of the operator $\mathcal{L}_{V}$ on $C \subset \mathbb{S}^{N-1}$ with homogeneous Dirichlet boundary conditions. Hence, if we denote by $\mu_{k_{0}}(V)$ the corresponding eigenvalue, it follows that $\varphi$ solves the equation

$$
-\varphi^{\prime \prime}(r)-\frac{N-1}{r} \varphi^{\prime}(r)+\frac{\mu_{k_{0}}(V)}{r^{2}} \varphi(r)=0 \quad \text { in }(0,1) .
$$

The general solution of the above equation is given by

$$
\varphi(r)=c_{1} r^{\sigma_{k_{0}}^{+}}+c_{2} r^{\sigma_{k_{0}}^{-}}, \quad c_{1}, c_{2} \in \mathbb{R},
$$

where $\sigma_{k_{0}}^{ \pm}=-\frac{N-2}{2} \pm \sqrt{\left(\frac{N-2}{2}\right)^{2}+\mu_{k_{0}}(V)}$. We observe that the function $|x|^{\sigma_{k_{0}}^{-}} \psi\left(\frac{x}{|x|}\right) \notin H^{1}\left(B_{1}\right)$ and hence $c_{2}=0$. Moreover $\varphi(1)=1$ implies $c_{1}=1$, so that $\widetilde{w}$ takes the form $\widetilde{w}(r, \theta)=r^{\sigma_{k_{0}}^{+}} \psi(\theta)$. Finally, inserting this representation of $\widetilde{w}$ in $\mathcal{N}_{\widetilde{w}}$ and taking into account that

$$
\mathcal{N}_{\widetilde{w}}(r)=\frac{D_{\widetilde{w}}(r)}{H_{\widetilde{w}}(r)}=\frac{r}{2} \frac{H_{\widetilde{w}}^{\prime}(r)}{H_{\widetilde{w}}(r)}=\frac{r \int_{\mathcal{C} \cap \partial B_{r}} \frac{\partial \widetilde{w}}{\partial \nu} \widetilde{w} d \sigma}{\int_{\mathcal{C} \cap \partial B_{r}} \widetilde{w}^{2} d \sigma},
$$

from (169) it follows that $\sigma_{k_{0}}^{+}=\gamma$. The proof is thereby complete.

We now study the behavior of $H(\lambda)$ as $\lambda \rightarrow 0^{+}$.

Lemma 6.2. Let $\gamma$ as in Lemmas 5.11 and 5.19. Then

$$
\lim _{\lambda \rightarrow 0^{+}} \lambda^{-2 \gamma} H(\lambda)
$$

exists and is finite.

Proof. In view of (119), (137), Lemma 5.5(i) and Lemma 5.14(i), it is sufficient to prove that the limit exists. By (85) and Lemmas 5.11, 5.19 we have

$$
\begin{aligned}
\frac{d}{d r} \frac{H(r)}{r^{2 \gamma}} & =-2 \gamma r^{-2 \gamma-1} H(r)+r^{-2 \gamma} H^{\prime}(r) \\
& =2 r^{-2 \gamma-1}\left(D(r)-\gamma H(r)+H(r) O\left(r^{\delta}\right)\right)=2 r^{-2 \gamma-1} H(r)\left(\int_{0}^{r} \mathcal{N}^{\prime}(s) d s+O\left(r^{\delta}\right)\right)
\end{aligned}
$$

Let us define the functions

$$
\begin{aligned}
& \nu_{1}(r):=\frac{\int_{\Gamma_{r}} \frac{|\nabla w|^{2}(\tilde{A} \tilde{\nu} \cdot \tilde{\nu})(\widetilde{A} x \cdot \tilde{\nu})}{\mu} d \sigma}{\int_{S_{r}} \mu w^{2} d \sigma} \\
&+\frac{2 r\left[\left(\int_{S_{r}} \frac{|\widetilde{A} \nabla w \cdot \nu|^{2}}{\mu} d \sigma\right)\left(\int_{S_{r}} \mu w^{2} d \sigma\right)-\left(\int_{S_{r}}(\widetilde{A} \nabla w \cdot \nu) w d \sigma(y)\right)^{2}\right]}{\left(\int_{S_{r}} \mu w^{2} d \sigma\right)^{2}} \\
& \nu_{2}(r):=\mathcal{N}^{\prime}(r)-\nu_{1}(r) .
\end{aligned}
$$

By Lemma 2.6 and Schwartz inequality we have that $\nu_{1} \geqslant 0$. On the other hand from the proofs of Lemmas 5.11, 5.19, 5.8, 5.16, we infer that the function $\nu_{2}$ is $L^{1}$-integrable in a right neighborhood of zero and moreover

$$
\int_{0}^{r} \nu_{2}(s) d s=O\left(r^{\hat{\delta}}\right), \quad \text { as } r \rightarrow 0^{+}
$$

where

$$
\hat{\delta}= \begin{cases}\min \left\{\delta,\left(q-2^{*}\right) / q\right\}, & \text { if } N \geqslant 3, \\ \min \left\{\delta, \frac{4}{p}, \frac{p-2}{2 p}\right\}, & \text { if } N=2,\end{cases}
$$


with $p$ as in (17) and $q$ as in (86). After integration of (171) over the interval $\left(r, r_{1}\right)$ we obtain

$$
\begin{aligned}
\frac{H\left(r_{1}\right)}{r_{1}^{2 \gamma}}-\frac{H(r)}{r^{2 \gamma}}= & \int_{r}^{r_{1}} 2 s^{-2 \gamma-1} H(s)\left(\int_{0}^{s} \nu_{1}(t) d t\right) d s+\int_{r}^{r_{1}} 2 s^{-2 \gamma-1} H(s)\left(\int_{0}^{s} \nu_{2}(t) d t\right) d s \\
& +O\left(\int_{r}^{r_{1}} s^{-2 \gamma-1+\delta} H(s) d s\right) .
\end{aligned}
$$

From the nonnegativity of $\nu_{1}$ the $\operatorname{limit}_{\lim _{r \rightarrow 0^{+}}} \int_{r}^{r_{1}} 2 s^{-2 \gamma-1} H(s)\left(\int_{0}^{s} \nu_{1}(t) d t\right) d s$ exists. On the other hand, by (172), (119), (137)

$$
\left|s^{-2 \gamma-1} H(s)\left(\int_{0}^{s} \nu_{2}(t) d t\right)\right| \leqslant O(1) s^{-1}\left|\int_{0}^{s} \nu_{2}(t) d t\right|=O\left(s^{-1+\hat{\delta}}\right) \quad \text { as } s \rightarrow 0^{+}
$$

which proves that $s^{-2 \gamma-1} H(s)\left(\int_{0}^{s} \nu_{2}(t) d t\right)$ is $L^{1}$-integrable in a right neighborhood of the origin. From (119) and (137) it follows that $s^{-2 \gamma-1+\delta} H(s)=O\left(s^{-1+\delta}\right)$ as $s \rightarrow 0^{+}$and hence $s^{-2 \gamma-1+\delta} H(s)$ is $L^{1}$-integrable in a right neighborhood of the origin. We may therefore conclude that all terms in the right hand side of (174) admit a limit as $r \rightarrow 0^{+}$thus completing the proof of the lemma.

\section{Straightening the Domain}

Lemma 7.1. There exists $\widehat{R} \in(0, \widetilde{R})$ such that the function

$$
\begin{aligned}
& \Xi: \widetilde{\Omega} \cap B_{\widehat{R}} \rightarrow \mathcal{C} \cap B_{\widehat{R}}, \\
& \Xi(y)=\Xi\left(y^{\prime}, y_{N}\right)=\frac{\left(y^{\prime}, y_{N}-\widetilde{\varphi}\left(y^{\prime}\right)+\varphi_{0}\left(y^{\prime}\right)\right)}{\sqrt{1+\frac{\left(\varphi_{0}\left(y^{\prime}\right)-\widetilde{\varphi}\left(y^{\prime}\right)\right)^{2}+2 y_{N}\left(\varphi_{0}\left(y^{\prime}\right)-\widetilde{\varphi}\left(y^{\prime}\right)\right)}{\left|y^{\prime}\right|^{2}+y_{N}^{2}}}}
\end{aligned}
$$

is invertible. Furthermore, putting $\Phi=\Xi^{-1}$, we have

$$
\begin{aligned}
& \Phi \in C^{1}\left(\mathcal{C} \cap B_{\widehat{R}}, \widetilde{\Omega} \cap B_{\widehat{R}}\right), \quad \Phi^{-1} \in C^{1}\left(\widetilde{\Omega} \cap B_{\widehat{R}}, \mathcal{C} \cap B_{\widehat{R}}\right), \\
& \Phi\left(\mathcal{C} \cap \partial B_{r}\right)=\widetilde{\Omega} \cap \partial B_{r} \quad \text { for all } r \in(0, \widehat{R}), \\
& \Phi(x)=x+O\left(|x|^{1+\delta}\right) \quad \text { and } \quad \operatorname{Jac} \Phi(x)=\operatorname{Id}_{N}+O\left(|x|^{\delta}\right) \quad \text { as }|x| \rightarrow 0, \\
& \Phi^{-1}(y)=y+O\left(|y|^{1+\delta}\right) \quad \text { and } \quad \operatorname{Jac} \Phi^{-1}(y)=\operatorname{Id}_{N}+O\left(|y|^{\delta}\right) \quad \text { as }|y| \rightarrow 0, \\
& \operatorname{det} \operatorname{Jac} \Phi(x)=1+O\left(|x|^{\delta}\right) \quad \text { as }|x| \rightarrow 0 .
\end{aligned}
$$

Proof. It follows from the Local Inversion Theorem, (34-35), and direct calculations.

Let $u \in H^{1}(\Omega)$ be a weak solution to (19), so that $w=u \circ \Psi \in H^{1}(\widetilde{\Omega})$ weakly solves (37). Then

$$
v=w \circ \Phi=u \circ \Psi \circ \Phi \in H^{1}\left(\mathcal{C} \cap B_{\widehat{R}}\right)
$$

is a weak solution to

$$
\begin{cases}-\operatorname{div}(\widehat{A}(x) \nabla v(x))+\widehat{\mathbf{b}}(x) \cdot \nabla v(x)-\frac{V\left(\frac{x}{|x|}\right)}{|x|^{2}} v(x)=\widehat{h}(x) v(x)+\widehat{f}(x, v(x)), & \text { in } \mathcal{C} \cap B_{\widehat{R}} \\ v=0, & \text { on } \partial \mathcal{C} \cap B_{\widehat{R}}\end{cases}
$$

where

$$
\begin{aligned}
& \widehat{A}(x)=|\operatorname{det} \operatorname{Jac} \Phi(x)|(\operatorname{Jac} \Phi(x))^{-1} \widetilde{A}(\Phi(x))\left((\operatorname{Jac} \Phi(x))^{T}\right)^{-1}, \\
& \widehat{\mathbf{b}}(x)=|\operatorname{det} \operatorname{Jac} \Phi(x)| \widetilde{\mathbf{b}}(\Phi(x))\left((\operatorname{Jac} \Phi(x))^{T}\right)^{-1}, \\
& \widehat{f}(x, s)=|\operatorname{det} \operatorname{Jac} \Phi(x)| \tilde{f}(\Phi(x), s), \\
& \widehat{h}(x)=|\operatorname{detJac} \Phi(x)| \widetilde{h}(\Phi(x))+|\operatorname{det} J a c \Phi(x)|\left(\frac{V\left(\frac{\Phi(x)}{\Phi(x) \mid}\right)}{|\Phi(x)|^{2}}-\frac{V\left(\frac{x}{|x|}\right)}{|x|^{2}}\right)+(|\operatorname{det} \operatorname{Jac} \Phi(x)|-1) \frac{V\left(\frac{x}{|x|}\right)}{|x|^{2}} .
\end{aligned}
$$


By Lemmas 7.1, 2.4, and direct calculations, we obtain

$$
\begin{aligned}
& \widehat{A}(x)=\operatorname{Id}_{N}+O\left(|x|^{\delta}\right) \quad \text { as }|x| \rightarrow 0, \\
& \widehat{\mathbf{b}} \in L_{\mathrm{loc}}^{\infty}\left(\mathcal{C} \cap B_{\widehat{R}}, \mathbb{R}^{N}\right), \quad|\widehat{\mathbf{b}}(x)|=O\left(|x|^{-1+\delta}\right) \quad \text { as }|x| \rightarrow 0, \\
& \widehat{f} \in C^{0}\left(\left(\mathcal{C} \cap B_{\widehat{R}}\right) \times \mathbb{R}\right) \quad \text { and } \quad|\widehat{f}(x, s) s| \leqslant \begin{cases}C_{\widehat{f}}\left(|s|^{2}+|s|^{2^{*}}\right), & \text { if } N \geqslant 3, \\
C_{\widehat{f}}\left(|s|^{2}+|s|^{p}\right), & \text { if } N=2,\end{cases} \\
& \widehat{h} \in L_{\mathrm{loc}}^{\infty}\left(\mathcal{C} \cap B_{\widehat{R}}\right), \quad \widehat{h}(x)=O\left(|x|^{-2+\delta}\right) \quad \text { as }|x| \rightarrow 0 .
\end{aligned}
$$

Lemma 7.2. Let $H$ be as in (73) and $v=w \circ \Phi$ as in (181). Then

$$
\begin{aligned}
& H(\lambda)=\left(1+O\left(\lambda^{\delta}\right)\right) \int_{C} v^{2}(\lambda \theta) d \sigma(\theta), \\
& \frac{\int_{\mathcal{C} \cap B_{1}}\left|\nabla \hat{v}^{\lambda}(x)\right|^{2} d x}{H(\lambda)}=\left(1+O\left(\lambda^{\delta}\right)\right) \int_{\Omega_{\lambda} / \lambda}\left|\nabla w^{\lambda}(y)\right|^{2} d y=O(1),
\end{aligned}
$$

as $\lambda \rightarrow 0^{+}$, where $w^{\lambda}$ is defined in (140) and $\hat{v}^{\lambda}(x):=v(\lambda x)$.

Proof. From (177), by a change of variable

and

$$
H(\lambda)=\int_{C} \mu(\Phi(\lambda \theta)) v^{2}(\lambda \theta)|\operatorname{det} \operatorname{Jac} \Phi(\lambda \theta)| d \sigma(\theta)
$$

$$
\frac{\int_{\mathcal{C} \cap B_{1}}\left|\nabla \hat{v}^{\lambda}(x)\right|^{2} d x}{H(\lambda)}=\int_{\Omega_{\lambda} / \lambda}\left|\nabla w^{\lambda}(y) \operatorname{Jac} \Phi\left(\Phi^{-1}(\lambda y)\right)\right|^{2}\left|\operatorname{det} \operatorname{Jac} \Phi^{-1}(\lambda y)\right| d y
$$

for all $\lambda \in(0, \widehat{R})$. We conclude from $(75),(178-180)$, and $H^{1}$-boundedness of $\left\{w^{\lambda}\right\}$ (see the proof of Lemma 6.1).

Lemma 7.3. Let $v=w \circ \Phi$ be as in (181) and let $k_{0}$ and $\gamma$ as in Lemma 6.1(i). Then for every sequence $\lambda_{n} \rightarrow 0^{+}$there exist a subsequence $\lambda_{n_{k}}$ and $\psi \in H_{0}^{1}(C) \subset H^{1}\left(\mathbb{S}^{N-1}\right)$ eigenfunction of the operator $\mathcal{L}_{V}=-\Delta_{\mathbb{S}^{N-1}}-V$ associated to the eigenvalue $\mu_{k_{0}}(V)$ such that $\|\psi\|_{L^{2}\left(\mathbb{S}^{N-1}\right)}=1$, the convergences of $\frac{w\left(\lambda_{n_{k}} x\right)}{\sqrt{H\left(\lambda_{n_{k}}\right)}}$ to $|x|^{\gamma} \psi\left(\frac{x}{|x|}\right)$ stated in part ii) of Lemma 6.1 hold, and

$$
\frac{v\left(\lambda_{n_{k}} \cdot\right)}{\sqrt{\int_{C} v^{2}\left(\lambda_{n_{k}} \theta\right) d \sigma(\theta)}} \rightarrow \psi
$$

strongly in $L^{2}(C)$.

Proof. From Lemma 6.1, there exist a subsequence $\lambda_{n_{k}}$ and $\psi \in H_{0}^{1}(C) \subset H^{1}\left(\mathbb{S}^{N-1}\right)$ eigenfunction of the operator $\mathcal{L}_{V}=-\Delta_{\mathbb{S}^{N-1}}-V$ associated to the eigenvalue $\mu_{k_{0}}(V)$ such that $\|\psi\|_{L^{2}\left(\mathbb{S}^{N-1}\right)}=1$ and $\left(H\left(\lambda_{n_{k}}\right)\right)^{-1 / 2} w\left(\lambda_{n_{k}} x\right) \rightarrow|x|^{\gamma} \psi\left(\frac{x}{|x|}\right)$ in senses claimed in part ii) of Lemma 6.1, in particular strongly in $L^{2}\left(\mathbb{S}^{N-1}\right)$ and a.e. on $\mathbb{S}^{N-1}$. Moreover from $H^{1}$-boundedness of $w^{\lambda}$ (see the proof of Lemma 6.1) and (188) it follows that $\left\{\hat{v}^{\lambda} / \sqrt{H(\lambda)}\right\}_{\lambda}$ is bounded in $H^{1}\left(\mathcal{C} \cap B_{1}\right)$ and relatively compact in $L^{2}(C)$. Hence from (187) there exists $\widetilde{\psi} \in L^{2}(C)$ such that, up to a further subsequence,

$$
\frac{v\left(\lambda_{n_{k}} \cdot\right)}{\sqrt{\int_{C} v^{2}\left(\lambda_{n_{k}} \theta\right) d \sigma(\theta)}} \rightarrow \widetilde{\psi} \quad \text { in } L^{2}(C) \text { and a.e. }
$$

From (155) together with (120) and (138) which allow extending estimate (155) up to $\partial B_{1}$, we have that, in view of (178) and (187), for a.e. $\theta \in \mathbb{S}^{N-1}$,

$$
\begin{aligned}
\frac{v\left(\lambda_{n_{k}} \theta\right)}{\sqrt{\int_{C} v^{2}\left(\lambda_{n_{k}} \theta\right) d \sigma(\theta)}} & =\left(1+O\left(\lambda_{n_{k}}^{\delta}\right)\right) \frac{w\left(\Phi\left(\lambda_{n_{k}} \theta\right)\right)}{\sqrt{H\left(\lambda_{n_{k}}\right)}} \\
& =\left(1+O\left(\lambda_{n_{k}}^{\delta}\right)\right)\left[\frac{w\left(\lambda_{n_{k}} \theta\right)}{\sqrt{H\left(\lambda_{n_{k}}\right)}}+\left(w^{\lambda_{n_{k}}}\left(\frac{\Phi\left(\lambda_{n_{k}} \theta\right)}{\lambda_{n_{k}}}\right)-w^{\lambda_{n_{k}}}(\theta)\right)\right] \\
& =\frac{w\left(\lambda_{n_{k}} \theta\right)}{\sqrt{H\left(\lambda_{n_{k}}\right)}}+O\left(\lambda_{n_{k}}^{\delta}\right) \rightarrow \psi(\theta) \quad \text { as } k \rightarrow+\infty .
\end{aligned}
$$


Then $\widetilde{\psi}=\psi$ and the lemma is proved.

In the sequel we denote by $\psi_{i}$ a $L^{2}$-normalized eigenfunction of the operator $\mathcal{L}_{V}=-\Delta_{\mathbb{S}^{N-1}}-V$ on the spherical cap $C \subset \mathbb{S}^{N-1}$ under null Dirichlet boundary conditions associated to the $i$-th eigenvalue $\mu_{i}(V)$, i.e.

$$
\begin{cases}\mathcal{L}_{V} \psi_{i}(\theta)=\mu_{i}(V) \psi_{i}(\theta), & \text { in } C, \\ \psi_{i}=0, & \text { on } \partial C, \\ \int_{\mathbb{S}^{N-1}}\left|\psi_{i}(\theta)\right|^{2} d \sigma(\theta)=1 . & \end{cases}
$$

Moreover, we choose the $\psi_{i}$ 's in such a way that the set $\left\{\psi_{i}\right\}_{i \in \mathbb{N} \backslash\{0\}}$ forms an orthonormal basis of $L^{2}(C)$. For all $i \in \mathbb{N}, i \geqslant 1$, and $\lambda \in(0, \widehat{R})$, we also define

$$
\varphi_{i}(\lambda):=\int_{C} v(\lambda \theta) \psi_{i}(\theta) d \sigma(\theta) .
$$

From Lemma 6.1 , there exist $j_{0}, m \in \mathbb{N}, j_{0}, m \geqslant 1$ such that $m$ is the multiplicity of the eigenvalue $\mu_{j_{0}}(V)=\mu_{j_{0}+1}(V)=\cdots=\mu_{j_{0}+m-1}(V)$ and

$$
\gamma=\lim _{r \rightarrow 0^{+}} \mathcal{N}(r)=-\frac{N-2}{2}+\sqrt{\left(\frac{N-2}{2}\right)^{2}+\mu_{i}(V)}, \quad i=j_{0}, \ldots, j_{0}+m-1 .
$$

Let $\mathcal{E}_{0}$ be the eigenspace of the operator $\mathcal{L}_{V}$ associated to the eigenvalue $\mu_{j_{0}}(V)$, so that the set $\left\{\psi_{i}\right\}_{i=j_{0}, \ldots, j_{0}+m-1}$ is an orthonormal basis of $\mathcal{E}_{0}$.

Lemma 7.4. Let $v=w \circ \Phi$ be as in (181), $j_{0}$ and $m$ as in (191) and $\varphi_{i}$ as in (190). Then for all $i \in\left\{j_{0}, \ldots, j_{0}+m-1\right\}$ and $R \in(0, \widehat{R})$

$$
\begin{aligned}
\varphi_{i}(\lambda)=\lambda^{\gamma}\left(R^{-\gamma} \varphi_{i}(R)+\frac{2-N-\gamma}{2-N-2 \gamma} \int_{\lambda}^{R}\right. & s^{-N+1-\gamma} \Upsilon_{i}(s) d s \\
& \left.-\frac{\gamma R^{-N+2-2 \gamma}}{2-N-2 \gamma} \int_{0}^{R} s^{\gamma-1} \Upsilon_{i}(s) d s\right)+O\left(\lambda^{\gamma+\hat{\delta}}\right)
\end{aligned}
$$

as $\lambda \rightarrow 0^{+}$with $\hat{\delta}$ as in (173) and $\Upsilon_{i} \in L^{1}(0, \widehat{R})$ defined as

$$
\begin{aligned}
\Upsilon_{i}(\lambda)=- & \int_{\mathcal{C} \cap B_{\lambda}}\left(\widehat{A}(x)-\operatorname{Id}_{N}\right) \nabla v(x) \\
& +\int_{\mathcal{C} \cap B_{\lambda}}(-\widehat{\mathbf{b}}(x) \cdot \nabla v(x)+\widehat{h}(x) v(x)+\widehat{f}(x, v(x))) \psi_{i}(x /|x|) d x \\
& +\int_{\mathcal{C} \cap \partial B_{\lambda}}\left(\widehat{A}(x)-\operatorname{Id}_{N}\right) \nabla v(x) \cdot \frac{x}{|x|} \psi_{i}(x /|x|) d \sigma(x) .
\end{aligned}
$$

Proof. For any $\lambda \in(0, \widehat{R})$, we expand $\theta \mapsto v(\lambda \theta) \in L^{2}(C)$ in Fourier series with respect to the orthonormal basis $\left\{\psi_{i}\right\}$ of $L^{2}\left(\mathbb{S}^{N-1}\right)$ defined in (189), i.e.

$$
v(\lambda \theta)=\sum_{i=1}^{\infty} \varphi_{i}(\lambda) \psi_{i}(\theta) \quad \text { in } L^{2}(C),
$$

with $\varphi_{i}$ is defined in (190). For all $i$, we consider the distribution $\zeta_{i}$ on $(0, \widehat{R})$ defined as

$$
\begin{aligned}
\mathcal{D}^{\prime}(0, \widehat{R}) & \left\langle\zeta_{i}, \omega\right\rangle_{\mathcal{D}(0, \widehat{R})} \\
& ={ }_{H^{-1}\left(B_{\widehat{R}} \cap \mathcal{C}\right)}\left\langle\operatorname{div}\left(\left(\widehat{A}-\operatorname{Id}_{N}\right) \nabla v\right)-\widehat{\mathbf{b}} \cdot \nabla v+\widehat{h} v+\widehat{f}(x, v), \frac{\omega(|x|)}{|x|^{N-1}} \psi_{i}(x /|x|)\right\rangle_{H_{0}^{1}\left(B_{\widehat{R}} \cap \mathcal{C}\right)}
\end{aligned}
$$

for all $\omega \in \mathcal{D}(0, \widehat{R})$. Letting $\Upsilon_{i}$ as in (193), by direct calculations we have that

$$
\Upsilon_{i}^{\prime}(\lambda)=\lambda^{N-1} \zeta_{i}(\lambda) \quad \text { in } \mathcal{D}^{\prime}(0, \widehat{R}) .
$$


On the other hand, from the definition of $\zeta_{i}$ and the fact that $v$ solves (182), it follows that, for all $i$, the function $\varphi_{i}$ defined in (190) solves

$$
-\varphi_{i}^{\prime \prime}(\lambda)-\frac{N-1}{\lambda} \varphi_{i}^{\prime}(\lambda)+\frac{\mu_{i}(V)}{\lambda^{2}} \varphi_{i}(\lambda)=\zeta_{i}(\lambda) \quad \text { in the sense of distributions in }(0, \widehat{R}),
$$

which can be also written as

$$
-\left(\lambda^{N-1+2 \sigma_{i}}\left(\lambda^{-\sigma_{i}} \varphi_{i}(\lambda)\right)^{\prime}\right)^{\prime}=\lambda^{N-1+\sigma_{i}} \zeta_{i}(\lambda) \text { in the sense of distributions in }(0, \widehat{R}),
$$

where

$$
\sigma_{i}=-\frac{N-2}{2}+\sqrt{\left(\frac{N-2}{2}\right)^{2}+\mu_{i}(V)}
$$

Let us fix $R \in(0, \widehat{R})$. Integrating by parts the right hand side and taking into account (195), we obtain that there exists $c_{i} \in \mathbb{R}$ (depending on $R$ ) such that

$$
\left(\lambda^{-\sigma_{i}} \varphi_{i}(\lambda)\right)^{\prime}=-\lambda^{-N+1-\sigma_{i}} \Upsilon_{i}(\lambda)-\sigma_{i} \lambda^{-N+1-2 \sigma_{i}}\left(c_{i}+\int_{\lambda}^{R} s^{\sigma_{i}-1} \Upsilon_{i}(s) d s\right)
$$

in the sense of distributions in $(0, R)$. In particular $\varphi_{i} \in W_{\text {loc }}^{1,1}(0, \widehat{R})$. A further integration yields

$$
\begin{gathered}
\varphi_{i}(\lambda)=\lambda^{\sigma_{i}}\left(R^{-\sigma_{i}} \varphi_{i}(R)+\int_{\lambda}^{R} s^{-N+1-\sigma_{i}} \Upsilon_{i}(s) d s\right) \\
\quad+\sigma_{i} \lambda^{\sigma_{i}} \int_{\lambda}^{R} s^{-N+1-2 \sigma_{i}}\left(c_{i}+\int_{s}^{R} t^{\sigma_{i}-1} \Upsilon_{i}(t) d t\right) d s \\
=\lambda^{\sigma_{i}}\left(R^{-\sigma_{i}} \varphi_{i}(R)+\frac{2-N-\sigma_{i}}{2-N-2 \sigma_{i}} \int_{\lambda}^{R} s^{-N+1-\sigma_{i}} \Upsilon_{i}(s) d s+\frac{\sigma_{i} c_{i} R^{-N+2-2 \sigma_{i}}}{2-N-2 \sigma_{i}}\right) \\
\quad+\frac{\sigma_{i} \lambda^{-N+2-\sigma_{i}}}{N-2+2 \sigma_{i}}\left(c_{i}+\int_{\lambda}^{R} t^{\sigma_{i}-1} \Upsilon_{i}(t) d t\right) .
\end{gathered}
$$

Let $j_{0}, m \in \mathbb{N}$ be as in (191), so that the eigenvalue $\mu_{j_{0}}(V)=\mu_{j_{0}+1}(V)=\cdots=\mu_{j_{0}+m-1}(V)$ has multiplicity $m$ and

$$
\gamma=\lim _{r \rightarrow 0^{+}} \mathcal{N}(r)=\sigma_{i}, \quad i=j_{0}, \ldots, j_{0}+m-1,
$$

see Lemma 6.1. Estimate (187) and the Parseval identity yield

$$
H(\lambda)=\left(1+O\left(\lambda^{\delta}\right)\right) \int_{C}|v(\lambda \theta)|^{2} d \sigma(\theta)=\left(1+O\left(\lambda^{\delta}\right)\right) \sum_{i=1}^{\infty}\left|\varphi_{i}(\lambda)\right|^{2}, \quad \text { for all } 0<\lambda \leqslant R .
$$

We claim that

$$
\Upsilon_{i}(\lambda)=O\left(\lambda^{N-2+\hat{\delta}+\sigma_{i}}\right) \text { for every } i \in\left\{j_{0}, \ldots, j_{0}+m-1\right\} \quad \text { as } \lambda \rightarrow 0^{+},
$$

with $\hat{\delta}$ defined in (173). Let us prove (200). By (119), (137), (183), (188), Hölder inequality and a change of variable we obtain

$$
\begin{aligned}
& \left|\int_{\mathcal{C} \cap B_{\lambda}}\left(\widehat{A}(x)-\operatorname{Id}_{N}\right) \nabla v(x) \cdot \frac{\nabla_{\mathbb{S}^{N-1}} \psi_{i}(x /|x|)}{|x|} d x\right| \\
& \leqslant O\left(\lambda^{N-2+\delta+\sigma_{i}}\right) \int_{{\mathcal{C} \cap B_{1}}}|x|^{\delta} \frac{\left|\nabla \hat{v}^{\lambda}(x)\right|}{\sqrt{H(\lambda)}} \frac{\left|\nabla_{\mathbb{S}^{N-1}} \psi_{i}(x /|x|)\right|}{|x|} d x \\
& \quad \leqslant O\left(\lambda^{N-2+\delta+\sigma_{i}}\right)\left(\frac{\int_{\mathcal{C} \cap B_{1}}\left|\nabla \hat{v}^{\lambda}(x)\right|^{2} d x}{H(\lambda)}\right)^{1 / 2}\left(\int_{\mathcal{C} \cap B_{1}}|x|^{2 \delta-2}\left|\nabla_{\mathbb{S}^{N-1}} \psi_{i}(x /|x|)\right|^{2} d x\right)^{1 / 2} \\
& \quad=O\left(\lambda^{N-2+\delta+\sigma_{i}}\right) \quad \text { as } \lambda \rightarrow 0^{+}
\end{aligned}
$$


Similarly, by a change of variable, (39), (42), (119), (137), and boundedness in $H^{1}\left(B_{1}\right)$ of the set $\left\{\widetilde{w}^{\lambda}\right\}_{\lambda}$ (see the proof of Lemma 6.1), we obtain

$$
\int_{\mathcal{C} \cap B_{\lambda}}(-\widehat{\mathbf{b}}(x) \cdot \nabla v(x)+\widehat{h}(x) v(x)) \psi_{i}(x /|x|) d x=O\left(\lambda^{N-2+\delta+\sigma_{i}}\right) \quad \text { as } \lambda \rightarrow 0^{+} .
$$

Moreover, (41), Proposition 4.1, (119), (137), and boundedness of $\left\{\widetilde{w}^{\lambda}\right\}_{\lambda}$ in $H^{1}\left(B_{1}\right)$ imply that

$$
\begin{aligned}
& \left|\int_{\mathcal{C} \cap B_{\lambda}} \widehat{f}(x, v(x)) \psi_{i}(x /|x|) d x\right| \leqslant \mathrm{const}\left|\int_{\Omega_{\lambda}} \tilde{f}(y, w(y)) \psi_{i}\left(\frac{\Phi^{-1}(y)}{\left|\Phi^{-1}(y)\right|}\right) d y\right| \\
& \quad \leqslant \operatorname{const} \int_{\Omega_{\lambda}}\left(|w(y)|+|w(y)|^{\tilde{p}-1}\right) d y \\
& \leqslant \operatorname{const} \lambda^{N} \sqrt{H(\lambda)}\left(\int_{\Omega_{\lambda} / \lambda}\left|w^{\lambda}(x)\right| d x+\int_{\Omega_{\lambda} / \lambda}|w(\lambda x)|^{\tilde{p}-2}\left|w^{\lambda}(x)\right| d x\right) \\
& \leqslant \operatorname{const} \lambda^{N+\sigma_{i}}\left(\left\|w^{\lambda}\right\|_{H^{1}\left(\Omega_{\lambda} / \lambda\right)}+\left(\int_{\Omega_{\lambda / \lambda}}|w(\lambda x)|^{\tilde{q}} d x\right)^{\frac{\tilde{p}-2}{\tilde{q}}}\left\|w^{\lambda}\right\|_{L^{\tilde{p}}\left(\Omega_{\lambda} / \lambda\right)}\right) \\
& \leqslant \operatorname{const} \lambda^{N+\sigma_{i}}\left(1+\lambda^{-N(\tilde{p}-2) / \tilde{q}}\right)=O\left(\lambda^{N-2+\sigma_{i}+\left(2-N \frac{\tilde{p}-2}{\tilde{q}}\right)}\right)=O\left(\lambda^{N-2+\hat{\delta}+\sigma_{i}}\right) \quad \text { as } \lambda \rightarrow 0^{+}
\end{aligned}
$$

where $\tilde{p}=2^{*}$ if $N \geqslant 3$ and $\tilde{p}=p$ with $p$ as in (17) if $N=2$, while $\tilde{q}=q$ with $q$ is as in (86) if $N \geqslant 3$ and $\tilde{q}=2 p$ if $N=2$, so that $2-N \frac{\tilde{p}-2}{\tilde{q}} \geqslant \hat{\delta}$. In order to estimate the boundary term in (193), we perform the change of variables $x=\Phi^{-1}(y)$ and $y=\lambda \theta$ to obtain

$$
\begin{aligned}
& \int_{\mathcal{C} \cap \partial B_{\lambda}}\left(\widehat{A}(x)-\operatorname{Id}_{N}\right) \nabla v(x) \cdot \frac{x}{|x|} \psi_{i}(x /|x|) d \sigma(x) \\
& \left.=\lambda^{N-1} \int_{C_{\lambda}}\left(\widehat{A}\left(\Phi^{-1}(\lambda \theta)\right)\right)-\operatorname{Id}_{N}\right)\left(\nabla w(\lambda \theta) \operatorname{Jac} \Phi\left(\Phi^{-1}(\lambda \theta)\right)\right)^{T} \cdot \frac{\Phi^{-1}(\lambda \theta)}{\left|\Phi^{-1}(\lambda \theta)\right|} \times \\
& \quad \times \psi_{i}\left(\frac{\Phi^{-1}(\lambda \theta)}{\left|\Phi^{-1}(\lambda \theta)\right|}\right)\left|\operatorname{det} \operatorname{Jac} \Phi^{-1}(\lambda \theta)\right| d \sigma(\theta)
\end{aligned}
$$

and from this, using (178), (179), (180), (183), (119), (137), (120), (138), and (157), we arrive to

$$
\begin{aligned}
\mid \int_{\mathcal{C} \cap \partial B_{\lambda}} & \left(\widehat{A}(x)-\operatorname{Id}_{N}\right) \nabla v(x) \cdot \frac{x}{|x|} \psi_{i}(x /|x|) d \sigma(x) \mid \\
& \leqslant O\left(\lambda^{N-2+\delta+\sigma_{i}}\right)\left(\int_{C_{\lambda}}\left|\nabla w^{\lambda}(\theta)\right|^{2} d \sigma(\theta)\right)^{1 / 2}\left(\int_{C_{\lambda}}\left|\psi_{i}\left(\frac{\Phi^{-1}(\lambda \theta)}{\left|\Phi^{-1}(\lambda \theta)\right|}\right)\right|^{2} d \sigma(\theta)\right)^{1 / 2} \\
& =O\left(\lambda^{N-2+\delta+\sigma_{i}}\right) .
\end{aligned}
$$

Inserting (201), (202), (203), (204) into (193), the proof of (200) follows.

In the rest of the proof it is not restrictive to assume that $\sigma_{i} \neq 0$, since otherwise the proof of the lemma follows immediately from (197). From (200) we deduce that the map

$$
s \mapsto s^{-N+1-\sigma_{i}} \Upsilon_{i}(s) \in L^{1}(0, R)
$$

so that

$$
\begin{aligned}
\lambda^{\sigma_{i}}\left(R^{-\sigma_{i}} \varphi_{i}(R)+\frac{2-N-\sigma_{i}}{2-N-2 \sigma_{i}} \int_{\lambda}^{R} s^{-N+1-\sigma_{i}} \Upsilon_{i}(s) d s+\frac{\sigma_{i} c_{i} R^{-N+2-2 \sigma_{i}}}{2-N-2 \sigma_{i}}\right) & \\
& =O\left(\lambda^{\sigma_{i}}\right)=o\left(\lambda^{-N+2-\sigma_{i}}\right) \quad \text { as } \lambda \rightarrow 0^{+} .
\end{aligned}
$$

On the other hand, by (200) we also have that $t \mapsto t^{\sigma_{i}-1} \Upsilon_{i}(t) \in L^{1}(0, R)$. We now claim that

$$
c_{i}+\int_{0}^{R} t^{\sigma_{i}-1} \Upsilon_{i}(t) d t=0 .
$$

Suppose by contradiction that (207) is not true. Then, by (197) and (206) we infer

$$
\varphi_{i}(\lambda) \sim \frac{\sigma_{i}}{N-2+2 \sigma_{i}}\left(c_{i}+\int_{0}^{R} t^{\sigma_{i}-1} \Upsilon_{i}(t) d t\right) \lambda^{-N+2-\sigma_{i}} \quad \text { as } \lambda \rightarrow 0^{+} .
$$


If $N \geqslant 3$, Hardy inequality and the fact that $v \in H^{1}\left(\mathcal{C} \cap B_{R}\right)$ imply

$$
\int_{0}^{R} \lambda^{N-3}\left|\varphi_{i}(\lambda)\right|^{2} d \lambda \leqslant \int_{0}^{R} \lambda^{N-3}\left(\int_{C}|v(\lambda \theta)|^{2} d \sigma(\theta)\right) d \lambda=\int_{\mathcal{C} \cap B_{R}} \frac{|v(x)|^{2}}{|x|^{2}} d x<+\infty
$$

thus contradicting (208). If $N=2,(208),(196)$, and the fact we are assuming $\sigma_{i} \neq 0$ would imply

$$
\lim _{\lambda \rightarrow 0^{+}}\left|\varphi_{i}(\lambda)\right|=+\infty
$$

and, in turn, by (199) we would have

$$
\lim _{\lambda \rightarrow 0^{+}} H(\lambda)=+\infty
$$

in contradiction with (137). Claim (207) is thereby proved. By (197) and (207) we then obtain

$$
\begin{aligned}
\varphi_{i}(\lambda)= & \lambda^{\sigma_{i}}\left(R^{-\sigma_{i}} \varphi_{i}(R)+\frac{2-N-\sigma_{i}}{2-N-2 \sigma_{i}} \int_{\lambda}^{R} s^{-N+1-\sigma_{i}} \Upsilon_{i}(s) d s+\frac{\sigma_{i} c_{i} R^{-N+2-2 \sigma_{i}}}{2-N-2 \sigma_{i}}\right) \\
& -\frac{\sigma_{i} \lambda^{-N+2-\sigma_{i}}}{N-2+2 \sigma_{i}} \int_{0}^{\lambda} t^{\sigma_{i}-1} \Upsilon_{i}(t) d t .
\end{aligned}
$$

The proof the lemma follows inserting (207) into (209) and observing that by (200)

as $\lambda \rightarrow 0^{+}$.

$$
\frac{\sigma_{i} \lambda^{-N+2-\sigma_{i}}}{N-2+2 \sigma_{i}} \int_{0}^{\lambda} t^{\sigma_{i}-1} \Upsilon_{i}(t) d t=O\left(\lambda^{\sigma_{i}+\hat{\delta}}\right)
$$

The asymptotic behavior of $H(\lambda)$ as $\lambda \rightarrow 0^{+}$is evaluated in the following lemma.

Lemma 7.5. Let $H$ be as in (73) and let $\gamma$ be as in Lemmas 5.11 and 5.19 respectively in the cases $N \geqslant 3$ and $N=2$. Then

$$
\lim _{\lambda \rightarrow 0^{+}} \lambda^{-2 \gamma} H(\lambda)>0 .
$$

Proof. The fact that the limit in (210) exists and is finite was proved in Lemma 6.2 and hence we may proceed by contradiction by supposing that $\lim _{\lambda \rightarrow 0^{+}} \lambda^{-2 \gamma} H(\lambda)=0$. Let $j_{0}$ and $m$ be as in (191) and $\varphi_{i}$ as in (190). From (199) we deduce that for any $i \in\left\{j_{0}, \ldots, j_{0}+m-1\right\}$

$$
\lim _{\lambda \rightarrow 0^{+}} \lambda^{-\gamma} \varphi_{i}(\lambda)=0 .
$$

Therefore by Lemma 7.4, (198), and (205) we obtain

$$
R^{-\gamma} \varphi_{i}(R)-\frac{\gamma R^{-N+2-2 \gamma}}{2-N-2 \gamma} \int_{0}^{R} s^{\gamma-1} \Upsilon_{i}(s) d s=-\frac{2-N-\gamma}{2-N-2 \gamma} \int_{0}^{R} s^{-N+1-\gamma} \Upsilon_{i}(s) d s
$$

which replaced in (192) yields

$$
\varphi_{i}(\lambda)=-\frac{2-N-\gamma}{2-N-2 \gamma} \lambda^{\gamma} \int_{0}^{\lambda} s^{-N+1-\gamma} \Upsilon_{i}(s) d s+O\left(\lambda^{\gamma+\hat{\delta}}\right) \quad \text { as } \lambda \rightarrow 0^{+} .
$$

Inserting in the last estimate (200) we conclude that for any $i \in\left\{j_{0}, \ldots, j_{0}+m-1\right\}$

$$
\varphi_{i}(\lambda)=O\left(\lambda^{\gamma+\hat{\delta}}\right) \quad \text { as } \lambda \rightarrow 0^{+} .
$$

This, together with (190), implies

$$
\int_{C} v(\lambda \theta) \phi(\theta) d \sigma(\theta)=O\left(\lambda^{\gamma+\hat{\delta}}\right) \quad \text { as } \lambda \rightarrow 0^{+}
$$

for any function $\phi$ in the eigenspace $\mathcal{E}_{0}$. By (211), (187), (121) in the case $N \geqslant 3$, and (139) with $\sigma=\hat{\delta}$ in the case $N=2$, we obtain

$$
\int_{C} \frac{v(\lambda \theta)}{\left\|\hat{v}^{\lambda}\right\|_{L^{2}(C)}} \phi(\theta) d \sigma(\theta)=O\left(\lambda^{\hat{\delta} / 2}\right)=o(1) \quad \text { as } \lambda \rightarrow 0^{+}
$$

for any $\phi \in \mathcal{E}_{0}$. On the other hand, Lemma 7.3 states that for any sequence $\lambda_{n} \rightarrow 0^{+}$there exists a subsequence $\lambda_{n_{k}}$ and a function $\psi \in \mathcal{E}_{0}$ with $\|\psi\|_{L^{2}(C)}=1$ such that

$$
\frac{v\left(\lambda_{n_{k}} \cdot\right)}{\left\|\hat{v}^{\lambda_{n_{k}}}\right\|_{L^{2}(C)}} \rightarrow \psi
$$


strongly in $L^{2}(C)$. Therefore, taking $\phi=\psi$ in (212) we conclude that

$$
0=\lim _{k \rightarrow+\infty}\left(\frac{\hat{v}^{\lambda_{n_{k}}}}{\left\|\hat{v}^{\lambda_{n_{k}}}\right\|_{L^{2}(C)}}, \psi\right)_{L^{2}(C)}=\|\psi\|_{L^{2}(C)}^{2}=1
$$

thus giving rise to a contradiction.

The following theorem is a more precise and complete version of Theorem 1.4.

Theorem 7.6. Let $\widetilde{A}, \widetilde{\mathbf{b}}, \tilde{f}, \widetilde{h}$ be as in (23-26) with $A, \mathbf{b}, \Psi, f, h, V$ as in assumptions (10-17), (21). Let $\widetilde{\Omega}$ be as in (29) with $\Omega$ satisfying (9) and (2-6). Let $w \in H^{1}(\widetilde{\Omega}) \backslash\{0\}$ be a non-trivial weak solution to (37). Then, letting $\mathcal{N}(r)$ as in (110) and (134), there exists $k_{0} \in \mathbb{N}, k_{0} \geqslant 1$, such that

$$
\lim _{r \rightarrow 0^{+}} \mathcal{N}(r)=-\frac{N-2}{2}+\sqrt{\left(\frac{N-2}{2}\right)^{2}+\mu_{k_{0}}(V)} .
$$

Furthermore, if $\gamma$ denotes the limit in (213), $m \geqslant 1$ is the multiplicity of the eigenvalue $\mu_{k_{0}}(V)$ and $\left\{\psi_{i}: j_{0} \leqslant i \leqslant j_{0}+m-1\right\}\left(j_{0} \leqslant k_{0} \leqslant j_{0}+m-1\right)$ is an $L^{2}(C)$-orthonormal basis for the eigenspace associated to $\mu_{k_{0}}(V)$, then, denoting again as $w$ its trivial extension outside $\widetilde{\Omega}$,

$$
\lambda^{-\gamma} w(\lambda x) \rightarrow|x|^{\gamma} \sum_{i=j_{0}}^{j_{0}+m-1} \beta_{i} \psi_{i}\left(\frac{x}{|x|}\right) \quad \text { as } \lambda \rightarrow 0^{+}
$$

in $H^{1}\left(B_{1}\right)$ and in $C_{\text {loc }}^{1, \alpha}\left(\mathcal{C} \cap B_{1}\right)$ for any $\alpha \in(0,1)$, where

$$
\left(\beta_{j_{0}}, \beta_{j_{0}+1}, \ldots, \beta_{j_{0}+m-1}\right) \neq(0,0, \ldots, 0)
$$

and

$$
\beta_{i}=\int_{C} R^{-\gamma} w(\Phi(R \theta)) \psi_{i}(\theta) d \sigma(\theta)+\frac{1}{2-N-2 \gamma} \int_{0}^{R}\left(\frac{2-N-\gamma}{s^{N-1+\gamma}}-\gamma \frac{s^{\gamma-1}}{R^{N-2+2 \gamma}}\right) \Upsilon_{i}(s) d s,
$$

for all $R \in(0, \widehat{R})$ for some $\widehat{R}>0, \Upsilon_{i}$ being defined in (193).

Proof. Identity (213) follows immediately from Lemma 6.1. As in the statement of the theorem, let $m$ be the multiplicity of the eigenvalue $\mu_{k_{0}}(V)$ found in Lemma 6.1, $j_{0} \in \mathbb{N} \backslash\{0\}$, such that $j_{0} \leqslant k_{0} \leqslant j_{0}+m-1, \mu_{j_{0}}(V)=\mu_{j_{0}+1}(V)=\cdots=\mu_{j_{0}+m-1}(V)$, and $\gamma=\lim _{r \rightarrow 0^{+}} \mathcal{N}(r)$.

In order to prove (214), let $\left\{\lambda_{n}\right\}_{n \in \mathbb{N}} \subset(0, \infty)$ be a sequence such that $\lambda_{n} \rightarrow 0^{+}$as $n \rightarrow+\infty$. By Lemmas 6.1, 6.2, 7.3, 7.5, and (187), there exist a subsequence $\lambda_{n_{j}}$ and $\beta_{j_{0}}, \ldots, \beta_{j_{0}+m-1} \in \mathbb{R}$ such that $\left(\beta_{j_{0}}, \beta_{j_{0}+1}, \ldots, \beta_{j_{0}+m-1}\right) \neq(0,0, \ldots, 0)$,

$$
\lambda_{n_{j}}^{-\gamma} w\left(\lambda_{n_{j}} x\right) \rightarrow|x|^{\gamma} \sum_{i=j_{0}}^{j_{0}+m-1} \beta_{i} \psi_{i}\left(\frac{x}{|x|}\right) \quad \text { in } H^{1}\left(B_{1}\right) \text { and } C_{\text {loc }}^{1, \alpha}\left(\mathcal{C} \cap B_{1}\right) \text { for any } \alpha \in(0,1)
$$

(with $w$ meant to be trivially extended outside $\widetilde{\Omega}$ ), and

$$
\lambda_{n_{j}}^{-\gamma} v\left(\lambda_{n_{j}} \cdot\right) \rightarrow \sum_{i=j_{0}}^{j_{0}+m-1} \beta_{i} \psi_{i} \quad \text { in } L^{2}(C) \quad \text { as } j \rightarrow+\infty .
$$

We now prove that the $\beta_{i}$ 's depend neither on the sequence $\left\{\lambda_{n}\right\}_{n \in \mathbb{N}}$ nor on its subsequence $\left\{\lambda_{n_{j}}\right\}_{j \in \mathbb{N}}$. Let us fix $R \in(0, \widehat{R})$ with $\widehat{R}$ as in Lemma 7.1. Defining $\varphi_{i}$ as in (190), from (217) it follows that, for any $i=j_{0}, \ldots, j_{0}+m-1$,

$$
\lambda_{n_{j}}^{-\gamma} \varphi_{i}\left(\lambda_{n_{j}}\right)=\int_{C} \frac{v\left(\lambda_{n_{j}} \theta\right)}{\lambda_{n_{j}}^{\gamma}} \psi_{i}(\theta) d \sigma(\theta) \rightarrow \sum_{\ell=j_{0}}^{j_{0}+m-1} \beta_{\ell} \int_{\mathbb{S}^{N-1}} \psi_{\ell}(\theta) \psi_{i}(\theta) d \sigma(\theta)=\beta_{i}
$$

as $j \rightarrow+\infty$. On the other hand, from Lemma 7.4, it follows that, for any $i=j_{0}, \ldots, j_{0}+m-1$,

$$
\lambda^{-\gamma} \varphi_{i}(\lambda) \rightarrow R^{-\gamma} \varphi_{i}(R)+\frac{2-N-\gamma}{2-N-2 \gamma} \int_{0}^{R} s^{-N+1-\gamma} \Upsilon_{i}(s) d s-\frac{\gamma R^{-N+2-2 \gamma}}{2-N-2 \gamma} \int_{0}^{R} s^{\gamma-1} \Upsilon_{i}(s) d s
$$


as $\lambda \rightarrow 0^{+}$and therefore from (218) we deduce that

$$
\beta_{i}=R^{-\gamma} \varphi_{i}(R)+\frac{2-N-\gamma}{2-N-2 \gamma} \int_{0}^{R} s^{-N+1-\gamma} \Upsilon_{i}(s) d s-\frac{\gamma R^{-N+2-2 \gamma}}{2-N-2 \gamma} \int_{0}^{R} s^{\gamma-1} \Upsilon_{i}(s) d s,
$$

for any $i=j_{0}, \ldots, j_{0}+m-1$. In particular the $\beta_{i}$ 's depend neither on the sequence $\left\{\lambda_{n}\right\}_{n \in \mathbb{N}}$ nor on its subsequence $\left\{\lambda_{n_{k}}\right\}_{k \in \mathbb{N}}$, thus implying that the convergence in (216) actually holds as $\lambda \rightarrow 0^{+}$and proving the theorem.

Proof of Theorem 1.1. Let us first observe that the family of functions

$$
u^{\lambda}(x)=\frac{u(\lambda x)}{\sqrt{H(\lambda)}}
$$

is bounded in $H^{1}\left(B_{1}\right)$, where $u$ is meant to be trivially extended outside $\Omega$. Indeed, by the change of variable $\lambda x=\Psi(\lambda y)$

$$
\begin{aligned}
& \int_{B_{1}}\left|\nabla u^{\lambda}(x)\right|^{2} d x=\int_{\frac{\Psi-1\left(B_{\lambda}\right)}{\lambda}}\left|\nabla \widetilde{w}^{\lambda}(y)(\operatorname{Jac} \Psi(\lambda y))^{-1}\right|^{2}|\operatorname{det} \operatorname{Jac} \Psi(\lambda y)| d y, \\
& \int_{B_{1}}\left|u^{\lambda}(x)\right|^{2} d x=\int_{\frac{\Psi^{-1}\left(B_{\lambda}\right)}{\lambda}}\left|\widetilde{w}^{\lambda}(y)\right|^{2}|\operatorname{det} \operatorname{Jac} \Psi(\lambda y)| d y,
\end{aligned}
$$

and hence from (21), (120), (138), and boundedness in $H^{1}\left(B_{1}\right)$ of the set $\left\{\widetilde{w}^{\lambda}\right\}_{\lambda}$ it follows that $\left\{u^{\lambda}\right\}_{\lambda}$ is bounded in $H^{1}\left(B_{1}\right)$ uniformly with respect to $\lambda$. Hence we can repeat for $u^{\lambda}$ the same arguments performed in the proof of Lemma 6.1 for $w^{\lambda}$ to obtain that $\left\{u^{\lambda}\right\}_{\lambda}$ is relatively compact in $C_{\text {loc }}^{1, \alpha}\left(\mathcal{C} \cap B_{1}\right)$, in $C_{\text {loc }}^{0, \alpha}\left(B_{1} \backslash\{0\}\right)$, and in $H^{1}\left(B_{1}\right)$ and hence, by Lemma 7.5 ,

(219) $\left\{\lambda^{-\gamma} u(\lambda \cdot)\right\}_{\lambda}$ is relatively compact in $C_{\text {loc }}^{1, \alpha}\left(\mathcal{C} \cap B_{1}\right)$, in $C_{\text {loc }}^{0, \alpha}\left(B_{1} \backslash\{0\}\right)$, and in $H^{1}\left(B_{1}\right)$, with $\gamma$ as in Theorem 7.6. Furthermore, from Lemma 7.5, (21), and (155)

$$
\left|\lambda^{-\gamma} u(\lambda x)-\lambda^{-\gamma} w(\lambda x)\right|=\frac{\sqrt{H(\lambda)}}{\lambda^{\gamma}}\left(w^{\lambda}\left(\frac{\Psi^{-1}(\lambda x)}{\lambda}\right)-w^{\lambda}(x)\right) \rightarrow 0
$$

for all $x \in \mathcal{C} \cap B_{1}$. From the above limit and Theorem 7.6 we deduce that, for all $x \in \mathcal{C} \cap B_{1}$,

$$
\lambda^{-\gamma} u(\lambda x) \rightarrow|x|^{\gamma} \sum_{i=j_{0}}^{j_{0}+m-1} \beta_{i} \psi_{i}\left(\frac{x}{|x|}\right) \quad \text { as } \lambda \rightarrow 0^{+}
$$

with $\beta_{i}$ and $\psi_{i}$ as in Theorem 7.6. Combining (219) and (220) we deduce that

$$
\lambda^{-\gamma} u(\lambda x) \rightarrow|x|^{\gamma} \sum_{i=j_{0}}^{j_{0}+m-1} \beta_{i} \psi_{i}\left(\frac{x}{|x|}\right) \quad \text { as } \lambda \rightarrow 0^{+}
$$

in $C_{\text {loc }}^{1, \alpha}\left(\mathcal{C} \cap B_{1}\right)$, in $C_{\text {loc }}^{0, \alpha}\left(B_{1} \backslash\{0\}\right)$ for all $\alpha \in(0,1)$, and in $H^{1}\left(B_{1}\right)$, thus completing the proof.

\section{An exAmple}

In this section we show that the presence of a logarithmic term in the asymptotic expansion cannot be excluded without assuming conditions (4) and (5).

Let us consider a domain $\Omega \subset \mathbb{R}^{2}$ admitting a local representation in a neighborhood of the origin as in (9) where the corresponding function $\varphi$ satisfies (2),

$$
\sup _{\nu \in \mathbb{S}^{N-2}}\left|\frac{\varphi(t \nu)}{t}-g(\nu)\right|=o(1) \quad \text { as } t \rightarrow 0^{+},
$$

with $g$ as in (3), but not (4-5). To this purpose let us define in Gauss plane the sets

$$
A_{1}:=\mathbb{C} \backslash\left\{z=i x_{2} \in \mathbb{C}: x_{2} \leqslant 0\right\}, \quad A_{2}:=\mathbb{C} \backslash\left\{x_{1} \in \mathbb{R} \subset \mathbb{C}: x_{1} \leqslant 0\right\}
$$

and the holomorphic functions $\eta_{1}: A_{1} \rightarrow \mathbb{C}, \eta_{2}: A_{2} \rightarrow \mathbb{C}$ defined as follows:

$$
\begin{aligned}
& \eta_{1}(z):=\log r+i \theta \quad \text { for any } z=r e^{i \theta} \in A_{1}, r>0, \theta \in\left(-\frac{\pi}{2}, \frac{3 \pi}{2}\right), \\
& \eta_{2}(z):=\log r+i \theta \quad \text { for any } z=r e^{i \theta} \in A_{2}, r>0, \theta \in(-\pi, \pi) .
\end{aligned}
$$


Given $\alpha \in(0,2)$, we are going to define $\Omega$ in such a way that $\partial \Omega$ admits at 0 a corner with amplitude $\alpha \pi$. We distinguish the cases $\alpha \in(0,1)$, and $\alpha \in[1,2)$.

The case $\alpha \in(0,1)$. Let us consider the holomorphic function

$$
v_{1}(z):=e^{\frac{2}{\alpha} \eta_{1}(-i z)} \eta_{1}(-i z) \quad \text { for any } z \in\{w \in \mathbb{C}: \Im w>0\}
$$

and the set

$$
\mathcal{Z}_{1}:=\left\{z: \Im z>0 \text { and } \Im\left(v_{1}(z)\right)=0\right\} .
$$

If $z=r e^{i \theta}$ with $r>0, \theta \in(0, \pi) \backslash\left\{\frac{\pi}{2}\right\}$, then $z \in \mathcal{Z}_{1}$ if and only

$$
r=\rho_{1}(\theta):=\exp \left[-\left(\theta-\frac{\pi}{2}\right) \cot \left(\frac{2}{\alpha}\left(\theta-\frac{\pi}{2}\right)\right)\right] .
$$

For some fixed $\sigma \in\left(0, \frac{\pi}{2}(1-\alpha)\right)$, we define the curves $\gamma_{\sigma}^{+} \subset \mathcal{Z}_{1}$ and $\gamma_{\sigma}^{-} \subset \mathcal{Z}_{1}$ respectively parametrized by

$$
\gamma_{\sigma}^{+}:\left\{\begin{array}{l}
x_{1}(\theta)=\rho_{1}(\theta) \cos \theta \\
x_{2}(\theta)=\rho_{1}(\theta) \sin \theta
\end{array} \quad \theta \in\left(\frac{\pi}{2}-\frac{\alpha \pi}{2}-\sigma, \frac{\pi}{2}-\frac{\alpha \pi}{2}\right)\right.
$$

and

$$
\gamma_{\sigma}^{-}:\left\{\begin{array}{l}
x_{1}(\theta)=\rho_{1}(\theta) \cos \theta \\
x_{2}(\theta)=\rho_{1}(\theta) \sin \theta
\end{array} \quad \theta \in\left(\frac{\pi}{2}+\frac{\alpha \pi}{2}, \frac{\pi}{2}+\frac{\alpha \pi}{2}+\sigma\right) .\right.
$$

If we choose $\sigma>0$ sufficiently small then the union of these two curves is the graph of a function $\varphi$ defined in a neighborhood $U$ of 0 . Moreover $\varphi$ is a Lipschitz function in $U, \varphi \in C^{1}(U \backslash\{0\})$ and

$$
\lim _{t \rightarrow 0^{-}} \frac{\varphi(t)}{t}=\tan \left(\frac{\pi}{2}+\frac{\alpha \pi}{2}\right), \quad \lim _{t \rightarrow 0^{+}} \frac{\varphi(t)}{t}=\tan \left(\frac{\pi}{2}-\frac{\alpha \pi}{2}\right) .
$$

At this point it is possible to construct a bounded domain $\Omega \subset\{z \in \mathbb{C}: \Im z>0\}$ satisfying (9) for some $R>0$ sufficiently small. Then we define the harmonic function

$$
u\left(x_{1}, x_{2}\right):=\Im\left(v_{1}(z)\right) \quad \text { for any } z=x_{1}+i x_{2} \in \Omega .
$$

In polar coordinates the function $u$ reads

$$
u(r, \theta)=r^{\frac{2}{\alpha}}\left[(\log r) \sin \left(\frac{2}{\alpha}\left(\theta-\frac{\pi}{2}\right)\right)+\left(\theta-\frac{\pi}{2}\right) \cos \left(\frac{2}{\alpha}\left(\theta-\frac{\pi}{2}\right)\right)\right] .
$$

Since $\Omega$ is bounded, then $u \in H^{1}(\Omega)$. From (221-222) and (226) we deduce that $u$ vanishes on $\gamma_{\sigma}^{+} \cup \gamma_{\sigma}^{-}$and in particular on $\partial \Omega \cap B_{R}$.

Next we show that $\varphi$ does not satisfy (7) for any $C_{0}>0$. Since by $(222-224) \varphi$ is an even function, it is sufficient to study the behavior of $\varphi\left(x_{1}\right)-x_{1} \varphi^{\prime}\left(x_{1}\right)$ in a right neighborhood of zero. By (223) and the fact that $\alpha \in(0,1)$ we may assume that $\theta \in\left(0, \frac{\pi}{2}\right)$ and hence, if $x_{1}$ belongs to a sufficiently small right neighborhood of 0 , by $(222)$ we have

$$
\frac{1}{2} \log \left(x_{1}^{2}+\left(\varphi\left(x_{1}\right)\right)^{2}\right) \tan \left[\frac{2}{\alpha}\left(\arctan \left(\frac{\varphi\left(x_{1}\right)}{x_{1}}\right)-\frac{\pi}{2}\right)\right]+\arctan \left(\frac{\varphi\left(x_{1}\right)}{x_{1}}\right)-\frac{\pi}{2}=0 .
$$

By (225) and (227) we have that, as $x_{1} \rightarrow 0^{+}$,

$$
\tan \left[\frac{2}{\alpha}\left(\arctan \left(\frac{\varphi\left(x_{1}\right)}{x_{1}}\right)-\frac{\pi}{2}\right)\right]=-\frac{2 \arctan \left(\frac{\varphi\left(x_{1}\right)}{x_{1}}\right)-\pi}{\log \left(x_{1}^{2}+\left(\varphi\left(x_{1}\right)\right)^{2}\right)}=\frac{\alpha \pi}{2} \frac{1}{\log x_{1}}+o\left(\frac{1}{\log x_{1}}\right) .
$$

Differentiating both sides of (227) and multiplying by $x_{1}^{2}+\left(\varphi\left(x_{1}\right)\right)^{2}$ we obtain the identity

$$
\begin{aligned}
\left(x_{1}+\varphi\left(x_{1}\right) \varphi^{\prime}\left(x_{1}\right)\right) \tan \left[\frac{2}{\alpha}\right. & \left.\left(\arctan \left(\frac{\varphi\left(x_{1}\right)}{x_{1}}\right)-\frac{\pi}{2}\right)\right] \\
& +\left\{1+\frac{\log \left(x_{1}^{2}+\left(\varphi\left(x_{1}\right)\right)^{2}\right)}{\alpha \cos ^{2}\left[\frac{2}{\alpha}\left(\arctan \left(\frac{\varphi\left(x_{1}\right)}{x_{1}}\right)-\frac{\pi}{2}\right)\right]}\right\}\left(x_{1} \varphi^{\prime}\left(x_{1}\right)-\varphi\left(x_{1}\right)\right)=0
\end{aligned}
$$

and hence (225) and (228) yield

$$
x_{1} \varphi^{\prime}\left(x_{1}\right)-\varphi\left(x_{1}\right) \sim-\frac{\alpha^{2} \pi\left[1+\tan ^{2}\left(\frac{\pi}{2}-\frac{\alpha \pi}{2}\right)\right]}{4} \frac{x_{1}}{\log ^{2} x_{1}} \quad \text { as } x_{1} \rightarrow 0^{+} .
$$


This shows that $\varphi$ does not satisfy condition (7).

The case $\alpha \in[1,2)$. Let us consider the holomorphic function

and the set

$$
v_{2}(z):=e^{\frac{2}{\alpha} \eta_{2}(-i z)} \eta_{2}(-i z) \quad \text { for any } z \in \mathbb{C} \backslash\{i y: y \leqslant 0\}
$$

$$
\mathcal{Z}_{2}:=\left\{z \in \mathbb{C} \backslash\{i y: y \leqslant 0\}: \Im\left(v_{2}(z)\right)=0\right\} .
$$

Similarly to the previous case one may define two curves $\gamma_{\sigma}^{+}, \gamma_{\sigma}^{-}$and a corresponding function $\varphi$ whose graph coincides with $\gamma_{\sigma}^{+} \cup \gamma_{\sigma}^{-}$. Next one may also construct a bounded domain $\Omega$ satisfying (9) for a suitable choice of $R>0$ and define a harmonic function $u$ as

$$
u\left(x_{1}, x_{2}\right):=\Im\left(v_{2}(z)\right) \quad \text { for any } z=x_{1}+i x_{2} \in \Omega .
$$

Finally one can prove that the new function $\varphi$ satisfies (229).

Aknowledgements. The authors would like to thank Prof. Susanna Terracini for helpful comments and discussions.

\section{REFERENCES}

[1] V. Adolfsson and L. Escauriaza. $C^{1, \alpha}$ domains and unique continuation at the boundary. Comm. Pure Appl. Math. 50 (1997), 935-969.

[2] F. J. Jr. Almgren. $Q$ valued functions minimizing Dirichlet's integral and the regularity of area minimizing rectifiable currents up to codimension two. Bull. Amer. Math. Soc. 8 (1983), 327-328.

[3] C. Băcuţă, A. L. Mazzucato, V. Nistor and L. Zikatanov. Interface and mixed boundary value problems on $n$-dimensional polyhedral domains. Doc. Math. 15 (2010), 687-745.

[4] H. Brézis and T. Kato. Remarks on the Schrödinger operator with singular complex potentials. J. Math. Pures Appl. (9) $\mathbf{5 8}$ (1979), 137-151.

[5] M. Costabel and M. Dauge. Singularités d'arêtes pour les problèmes aux limites elliptiques. Journées "Équations aux Dérivées Partielles" (Saint-Jean-de-Monts, 1992), Exp. No. IV, 12 pp., École Polytech., Palaiseau, 1992.

[6] M. Dauge. Elliptic boundary value problems on corner domains. Smoothness and asymptotics of solutions. Lecture Notes in Mathematics 1341. Springer-Verlag, Berlin, 1988.

[7] V. Felli, A. Ferrero and S. Terracini. Asymptotic behavior of solutions to Schrödinger equations near an isolated singularity of the electromagnetic potential. J. Eur. Math. Soc. (JEMS) 13 (2011), 119-174.

[8] V. Felli, A. Ferrero and S. Terracini. On the behavior at collisions of solutions to Schrödinger equations with many-particle and cylindrical potentials. Discrete Contin. Dynam. Systems - A 32 (2012), 3895-3956.

[9] V. Felli, A. Ferrero and S. Terracini. A note on local asymptotics of solutions to singular elliptic equations via monotonicity methods. Milan Journal of Math. (2012), DOI 10.1007/s00032-012-0174-y.

[10] N. Garofalo and F.-H. Lin. Monotonicity properties of variational integrals, $A_{p}$ weights and unique continuation. Indiana Univ. Math. J. 35 (1986), 245-268.

[11] P. Grisvard. Behavior of the solutions of an elliptic boundary value problem in a polygonal or polyhedral domain. Numerical solution of partial differential equations, III (Proc. Third Sympos. (SYNSPADE), Univ. Maryland, College Park, Md., 1975), pp. 207-274. Academic Press, New York, 1976.

[12] B. Kawohl. On nonlinear mixed boundary value problems for second order elliptic differential equations on domains with corners. Proc. Roy. Soc. Edinburgh Sect. A 87 (1980/81), 35-51.

[13] V. A. Kondrat'ev and O. A. Oleĭnik. Boundary value problems for partial differential equations in nonsmooth domains. Uspekhi Mat. Nauk 38 (1983), 3-76.

[14] R. S. Lehman. Development of the mapping function at an analytic corner. Pacific J. Math. 7 (1957), 1437-1449.

[15] V. Liskevich, S. Lyakhova and V. Moroz. Positive solutions to singular semilinear elliptic equations with critical potential on cone-like domains. Adv. Differential Equations 11 (2006), 361-398.

[16] V. G. Maz'ja. Sobolev Spaces. Springer Series in Soviet Mathematics, Springer-Verlag, Berlin, 1985.

[17] V. Maz'ya, S. Nazarov and B. Plamenevskij. Asymptotic theory of elliptic boundary value problems in singularly perturbed domains. Vol. I. Operator Theory: Advances and Applications 111. Birkhäuser Verlag, Basel, 2000.

[18] X. Tao and S. Zhang. Weighted doubling properties and unique continuation theorems for the degenerate Schrödinger equations with singular potentials. J. Math. Anal. Appl. 339 (2008), 70-84.

[19] S. Terracini. On positive entire solutions to a class of equations with singular coefficient and critical exponent. Adv. Diff. Equa. 1 (1996), 241-264.

[20] P. Tolksdorf. On the Dirichlet problem for quasilinear equations in domains with conical boundary points. Comm. Partial Differential Equations 8 (1983), 773-817.

[21] Z.-Q. Wang and M. Zhu. Hardy inequalities with boundary terms. Electron. J. Differential Equations (2003), No. 43.

[22] W. Wasow. Asymptotic development of the solution of Dirichlet's problem at analytic corners. Duke Math. J. 24 (1957), 47-56.

[23] N. M. Wigley. Corner behavior of solutions of semilinear Dirichlet problems. Canad. J. Math. 37 (1985), 1025-1046.

[24] T. H. Wolff. A property of measures in $\mathbb{R}^{N}$ and an application to unique continuation. Geom. Funct. Anal. 2 (1992), 225-284. 
Università di Milano BicocCA,

Dipartimento di Matematica e Applicazioni,

Via Cozzi 53, 20125 Milano, Italy.

E-mail address: veronica.felli@unimib.it.

Università degli Studi del Piemonte Orientale,

Viale Teresa Michel 11, 15121 Alessandria, Italy.

E-mail address: alberto.ferrero@mfn.unipmn.it. 